\title{
Tapping to unfamiliar and highly syncopated rhythms: Modelling behaviour and cognitive mechanisms
}

\author{
Andrew J. Milne ${ }^{\mathrm{a}, *}$, Roger T. Dean ${ }^{\mathrm{a}}$, David Bulger ${ }^{\mathrm{b}}$ \\ ${ }^{a}$ The MARCS Institute for Brain, Behaviour and Development, Western Sydney University, Penrith, Australia \\ ${ }^{\mathrm{b}}$ Department of Mathematics and Statistics, Macquarie University, Sydney, Australia
}

\begin{abstract}
By modelling tapping behaviour - rhythm-level tapping accuracy and pulse-level tap probabilities, velocities, and timing errors - to 91 mainly unfamiliar and complex rhythms, we identify some novel cognitive mechanisms that may play a role in temporal prediction. This was achieved with multilevel Bayesian regression using many and mostly new predictors, each derived from the structure of the rhythm and each indicative of one or more underlying cognitive mechanisms (lossy encodings of the rhythm and mechanisms derived from them). Each periodic rhythm comprised isochronous pulses sounded by a cymbal, while a non-isochronous subset of these were also sounded by a piano tone to act as cues for synchronized tapping. This is the first synchronized tapping study to use such a wide variety of unfamiliar rhythms and the results show that, for these tricky rhythms, participants' taps are disrupted by unfamiliar period lengths and taps are guided by rather crude encodings of each rhythm: the density of rhythmic cues, their circular mean and variance, and recognizing common small patterns and the approximate positions of groups of cues. These lossy encodings are often counterproductive for discriminating between cued and uncued pulses and are quite different to mechanisms - such as metricization and emphasizing group boundaries - thought to guide tapping behaviours in learned and familiar rhythms.
\end{abstract}

Keywords: music cognition, rhythm, meter, synchronized tapping, well-formed rhythms

\section{$1 \quad$ Introduction}

Western music, including baroque/classical/romantic, jazz, rock, and pop, typically uses rhythms with isochronous accents grouped, hierarchically, in twos or threes. For example, in pop music, it would be common to hear the hierarchy of accents shown in Table 1. Such music is usually performed and composed under the expectation that analogous hierarchical meters - subjective accents or expectations - are induced in the listener's mind; indeed, the rhythmical "spice" of syncopation, where rhythmic accents occur off the beat, arises by virtue of the rhythm contradicting these metrical expectations.

Table 1. An example of a common hierarchy of musical accents in Western pop music. The top line shows a fast isochronous (eighth-note triplets) hi-hat cymbal; in the second line, these cymbal pulses are grouped into threes by, alternately, the kick and snare drums (quarter-notes). The kick and snare pattern, as a whole, implies a duple half-note division, which is further grouped by the harmony, which changes each bar. The line at the top shows how a musician might count out this rhythm (mentally or verbally), and the depth of the hierarchy suggests the level of metrical accent that is subjectively felt: strongest at " 1 ", then " 3 ", then " 2 " and " 4 ", then every "\&" and "a". Different rhythmic and metrical hierarchies involving duple and triple groupings of isochronous pulses are common in most genres of Western music.

\begin{tabular}{|c|c|c|c|c|c|c|c|c|c|c|c|c|}
\hline$\overline{\text { Count (beats) }}$ & "1" & "\&" & "a" & $" 2 "$ & "\&" & "a" & "3" & "\&" & "a" & "4" & "\&" & "a" \\
\hline$\overline{\text { Hi-hat (onset) }}$ & $x$ & $x$ & $x$ & $x$ & $x$ & $x$ & $x$ & $x$ & $x$ & $x$ & $x$ & $x$ \\
\hline Kick OR Snare (onset) & $x$ & & & $x$ & & & $x$ & & & $x$ & & \\
\hline Kick (onset) & $x$ & & & & & & $x$ & & & & & \\
\hline Harmony (change) & $x$ & & & & & & & & & & & \\
\hline
\end{tabular}


Isochronous and simple syncopated rhythms, and the meters they induce, have been widely studied in sensorimotor synchronization tasks (typically assessed by tapping experiments). These have found, for example, that participants typically accent certain cues according to how they are grouped (Essens \& Povel, 1985; Repp et al., 2005), they generally tap a little early (Aschersleben, 2002), ratios between long and short interonset intervals are often distorted when tapped (Fraisse, 1946; Repp et al., 2012), timing accuracy for slow rhythms improves when they are audibly subdivided (Repp, 2003), and that mechanisms related to adaptation (period and phase correction) and anticipation (extrapolation and perseveration) are used by performers to synchronize with each other and with gradually changing tempos (Harry \& Keller, 2019).

However, outside the broad generalities of the above genres, and in much non-Western music, more complex non-isochronous rhythms are common. For example, the rhythmic emphases in Balkan aksak music typically form extended repeating patterns of "long" $(L)$ and "short" $(s)$ interonset intervals like Lss (approximated by $\left(\begin{array}{lll}3 & 2 & 2\end{array}\right)$ •.」 • ; the numbers represent

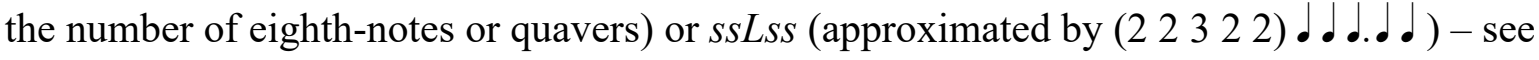
Fracile (2003) and Moelants (2006) for numerous other examples. In both these cases, the number of pulses in the cycle are prime numbers: 7 and 11, respectively, which cannot be factorized by either 2 or 3. High-prime rhythms like these are common in aksak whilst being comparatively rare in Western music. As noted by Toussaint $(2003 ; 2002)$, non-isochronous bell patterns are also characteristic of music originating from West Africa; one of the most notable of these being the Bembé, which has a form analogous to the equally tempered

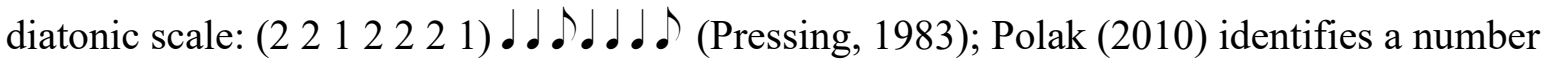
of distinct non-isochronous patterns used by Malian jembe drummers. Clayton (2020) provides a detailed analysis of how, in North Indian music, non-isochronous structures can pertain to even the deepest (slowest) metrical levels.

Furthermore, within the Western tradition, non-isochronous metrical or hypermetrical structures are a vital stylistic feature of jazz fusion, progressive and post-rock as exemplified by the (3 4 3) •.d •. pattern in Mahavishnu Orchestra's "The Dance of Maya" (McLaughlin, 1971); the (3 32 2) d.d.d . pattern in King Crimson's "Larks Tongues in Aspic, Part Two" (Fripp, 1973); the main (3 3331222223 ) riff (19 pulses) in Van der Graaf Generator's "Meurglys III" (Hammill, 1976); the (6 45332737348 ) cycle (of 55 pulses) running through much of Henry Cow's "Ruins" (Frith, 1974); and the widely discussed rhythm in Radiohead's "Pyramid Song" (Yorke et al., 2001), which can be approximated by (5 4654 ) (Hesselink, 2013; Osborn, 2017).

Complex non-isochronous rhythms such as these raise several pertinent questions: do they induce a regular meter (e.g., a regular metrical beat perceived to underlie the rhythm), or is this meter non-isochronous or, indeed, is no meter induced at all? What are the structural features (e.g., length, symmetries, complexity) of a rhythmic pattern that help or hinder the induction of a meter? How quickly can such rhythms be learned and what aspects of the rhythm influence this? How accurately can people tap along with such rhythms, and what cognitive mechanisms guide their tapping? Within each rhythm, which cues (the sounded events where they are meant to tap) are tapped more often, or with more accurate timing, or with greater tap velocity? By analogy, in the white-note diatonic scale, Western listeners rate the pitch class $\mathrm{C}$ as the best fitting or most stable (Hearne, 2020; Krumhans1, 1983) - might participants exhibit similar types of cognitive "bias" or "preference", exhibited in their tapping, for specific cues in non-isochronous rhythms? 
Despite their musical relevance and their theoretical interest, complex non-isochronous rhythms have had limited experimental investigation. Essens and Povel (1985) replicated spontaneous tapping experiments, originally conducted by Fraisse (1946, 1956), where participants were asked to tap a rhythm after a verbal description of a sequence of groups, each group specified by its number of cues. A "group" refers to a set of cues that are closely spaced in time and separated from other groups by a longer interval (in other words, they are clusters of cues). Intertap intervals within groups were approximately half the length of intertap intervals between groups, suggesting an attraction towards a within-group:betweengroup intertap ratio of 1:2 (Essens \& Povel, 1985).

In reproduction (continuation) tapping experiments, where participants reproduce a rhythm after it has stopped, Povel (1981), Povel and Essens (1985), Essens and Povel (1985) measured mean tap-timing errors (at the rhythm level) and ratio distortions of interonset intervals for a variety of uneven rhythms. They concluded that rhythms that conform more closely to an isochronous metrical grid are tapped more accurately. The underlying theory being that, when a rhythm is closely related to an isochronous grid, it can be more efficiently encoded whilst still keeping good temporal precision. In related findings, Collier and Wright (1995) showed that simple-ratio Ls rhythms can be rescaled (performed at a different tempo) better than those with complex ratios.

In our experiment, each periodic rhythm comprised isochronous pulses sounded by a cymbal, while a non-isochronous subset of these were also sounded by a piano tone to act as cues for synchronized tapping. We now discuss related non-isochronous synchronized tapping experiments. In addition to perception and reproduction tasks, Repp, London, and Keller $(2005,2011,2012,2013)$ used synchronized tapping tasks, where participants tapped along with a sounded rhythm, to investigate asynchronies and distortions of intertap ratios. These included both simple and extended uneven rhythms. They found tap ratios are typically distorted such that two-interval taps are attracted towards a ratio in the vicinity of 4:7, while three-interval taps seem attracted to two different attractor ratios. Snyder et al. (2006) used both synchronized and continuation taps for $\left(\begin{array}{lll}3 & 2 & 2\end{array}\right)$ and $\left(\begin{array}{lll}2 & 2 & 3\end{array}\right)$ rhythms (i.e., two different phases of the same rhythm), in a variety of melodic contexts, to assess tap ratios, tap timing variability and mean signed tap timing error. Timing variability was lower, and mean position was earlier, for the last tap in the cluster (the onset preceding the long IOI); the opposite for the first tap in the cluster. Like Repp et al., they found tap ratios distorted away from the correct 3:2 towards 2:1. Jones and Pfordresher (1997) measured timing errors of taps synchronized to non-isochronous melodic accents, although the timing errors (and variances) were taken across each rhythm as a whole rather than at the level of individual accents or taps. Polak et al. (2018) conducted a synchronization experiment on $s L$ rhythms with differing ratios performed by experienced musicians from Mali, Bulgaria, and Germany, finding cultural variations in the ratio distortions (ratios familiar to the performers were distorted less). Recorded aksak performances show similar distortions of simple 3:2 ratios (Goldberg, 2015). In sum, these tap ratio distortions, often away from pure integer ratios, seem prevalent. Jacoby and McDermott (2017) used an interesting paradigm where participants tapped as accurately as possible to a "seed" 3-onset rhythm with random IOIs (such that their sum was always 1 or 2 seconds); the tapped rhythm was then replicated and served as the next rhythm to be tapped to; this iteration occurred five times. The resulting rhythms clustered around simple integer ratios although, interestingly, the distributions differed between Tsimanè participants (a remote Bolivian community with minimal exposure to Western music) and US participants: a result that is clearly indicative of the importance of both integer-ratio musical universals and cultural exposure.

For complex and extended non-isochronous rhythms such as those exemplified above, we are aware of only two synchronized tapping studies modelling signed tap timing errors at a 
pulse, cue, or tap level (i.e., more finely grained than the mean tapping error across each rhythm): the aforementioned Repp et al., (2005) and Snyder et al., (2006). In both, it was found that successive cues in a group are tapped increasingly earlier. We are aware of only one previous study that has used synchronized tapping to non-isochronous cues to assess metrical accenting: In Repp et al. (2005), tap velocities were measured and were found to be correlated to the grouping accents identified by Povel (notably, the starts and ends of cue clusters were tapped harder than medial cues), as well as to the start point ("downbeat") of the rhythm.

In perceptual experiments, where no tapping took place, and participants matched the loudness of equal-intensity tones in $L s$ rhythms, Povel and Okkerman (1981) found that the longer the long IOI, the more likely the preceding cue would be perceived as accented (provided the short IOI is less than about $250 \mathrm{~ms}$ and the long IOI is noticeably larger). In Povel and Essens (1985), these findings (in addition to some unspecified pilot studies) were generalized into a set of principles: onsets at the ends of groups with one or more onsets, and at the starts of groups with three or more onsets, are subjectively accented.

Subjective metrical accents for uneven rhythms have been investigated by Parncutt (1994), who used a perceptual task with six different rhythmic patterns; participants rated how on- or off-beat (syncopated) each specific pulse in the rhythm sounded (the pulse to be rated was identified with a timbre change). As in our experiment, Parncutt included pulses that coincided with cues and those that did not. The results were modelled (nonlinearly) with a combination of predictors similar to those outlined above for Povel's (1981) study, as well as a pulse-match salience predictor derived (using our terminology) by summing weighted indicator vectors of all possible isochronous pulses, where the weighting is a quantification of how well that pulse matches the rhythm in question (this is related to the method used in Povel and Essens (1985) and almost identical to the subsequently developed autocorrelation phase (APM) matrix used by Eck (2006), which is one of the predictors - detailed in Section 2.2.3 - we use in our model). Parncutt's model had a very good fit to the data although, given its complexity and the small number of data points fitted (26), caution is advisable.

In summary, prior investigations of non-isochronous rhythms have focussed on tap-timing distortions of the ratios between long and short interonset intervals; only two have reported perceptual metrical accenting; only one has modelled performative accenting (tap velocity) by cue; only two have modelled signed tap timing errors by cue. We are not aware of any prior studies that have modelled tapping probabilities (e.g., the percentage of each rhythmic cue tapped). Most of these studies have used trained musician participants.

To advance understanding of the perception and production of complex and unfamiliar non-isochronous rhythms and to answer some of the questions raised earlier, we designed an experiment where mostly non-musician participants were asked to tap along with 91 different non-isochronous rhythms, the majority of which had extended structures, and all of whose cues are a subset of a regular pulse. We limited our selection of rhythms to a class of circular (periodic) patterns known as well-formed (or WF for short) (Carey \& Clampitt, 1989). Wellformed rhythms are defined and exemplified in Section 3.2: they allow a wide variety of extended patterns with just two distinct interonset intervals, which seems to be a common feature of many non-Western rhythms (e.g., the Euclidean/maximally-even rhythms identified by Toussaint (2002)), have interesting compositional possibilities (Milne, 2018; Milne \& Dean, 2016), and are defined for periodicities of any length (such as the familiar 3-, 4-, 6-, 8-, 9-, and 12-pulse periods, and the less familiar 5-, 7-, 10-, 11-, and 13-pulse periods).

The research questions related to the effects of rhythmic complexity on the learning of these rhythms are detailed in Dean et al. (in press). The focus in this article is to use a variety 
of structural features of complex non-isochronous rhythms (some of which vary by rhythm, some which vary by each pulse in each rhythm) to predict (a) the tapping accuracies associated with different rhythms, (b) how well taps discriminate between cued and uncued pulses, (c) tap velocities, and (d) tap timings relative to the pulses. Because many of these structural features are lossy compressed encodings of a rhythm - for example, its circular mean, or the edges of its clusters - they are indicative of underlying cognitive mechanisms related to memory storage and information processing. (By cognitive mechanisms we refer to specific processes involving perception, memory, learning, or decision-making, which transfer information between complex inputs and result in a spectrum of minimally flexible behavioural outputs (Newen, 2017); these processes may or may not be conscious.) Having said that, we cannot make definitive claims about underlying cognitive mechanisms or perception of meter because they are not directly tested in this study: strongly indicated mechanisms may be directly tested in future work. Although each structural feature is guided by an a priori directional hypothesis as to its effect on rhythm perception and production, this research is still somewhat exploratory, which motivates our use of an unusually large number of features, many of which are novel (and defined in Sec. 2.2).

As detailed later, the musical exposure of our participants is, despite their cultural diversity, strongly focussed on Western music or music with Western metrical structures. We have no way, therefore, to determine whether the features we find relevant are indicative of universal or cultural mechanisms; this kind of information is only obtainable through crosscultural studies, notably those on participants with minimal exposure to Western musical styles, as in Jacoby and McDermott (2017). Furthermore, because our focus is on the structural features of rhythms, we did not use predictors that track probabilities over the course of the experiment, nor over a corpus of culturally familiar music such as would be obtainable with the IDyOM model (Pearce, 2018), nor did we consider the influence of performative aspects such as varying loudnesses or timbres or on which cue the rhythm started (the former two were invariant and the latter was randomized, obviating any requirement for them to be modelled).

All data and code are available at https://osf.io/b8ad2/.

\section{Overview of variables and modelling}

Here, we detail the four response variables (Sec. 2.1); the predictors we use to model those responses and the cognitive mechanisms they each indicate (Sec. 2.2); and the multilevel Bayesian regression modelling approach we use to estimate these predictors' effects (Sec. 2.3).

\subsection{Dependent variables}

There are four dependent variables modelled in this study.

\subsubsection{Performance-level tap accuracy}

For each 30-second performance, tap_acc is a score summarising the timing accuracy of taps relative to cues as well as to the correctness of the tapping (i.e., correctly tapping on cued pulses or correctly refraining from tapping on uncued pulses results in higher accuracy than incorrectly tapping on uncued pulses or incorrectly refraining from tapping on cued pulses). It is calculated as the cosine similarity between a smoothed indicator vector of the taps and a smoothed indicator vector of the cues, both with a temporal resolution of $1 \mathrm{~ms}$. Gaussian smoothing of $10 \mathrm{~ms}$ standard deviation is used because this loosely approximates the temporal just noticeable difference for isochronous rhythms (Friberg \& Sundberg, 1995). We use this quantification, rather than mean asynchrony or the standard deviation of asynchronies, because it penalizes - in a principled way - "incorrect" taps that occur when there is no cue and missing taps when there is a cue; incorrect taps or missing taps are ignored when 
calculating asynchronies because of the requirement for a one-to-one pairing between every tap and every cue. This measure penalizes taps that occur early or late relative to a cue. This means it penalizes constant asynchrony; for example, a performance where every tap is exactly $30 \mathrm{~ms}$ before its cue will have lower accuracy than one where every tap is $15 \mathrm{~ms}$ before the cue. Our code allows for the effect of constant asynchrony to be factored out; however, we chose not to do so because constant asynchronies (e.g., negative mean asynchrony) are still inaccuracies; for example, professional drummers typically have lower negative mean asynchrony, sometimes approaching zero (Krause et al., 2010). Furthermore, for our data, the correlation between accuracy values achieved when factoring out constant offsets versus not factoring them out is 0.89 ; so, here, the two measures are quite similar. For reference, the standard deviation of tap-cue asynchronies in these data are reported in Dean et al. (in press). The tap_acc variable lies in the unit interval, hence is treated as beta distributed (conditional on the predictors). This novel method for calculating tap accuracy, and its useful properties, are detailed in Appendix A. 1.

\subsubsection{Tap probability}

For this and the following dependent variables, a tap is deemed to have occurred at any given pulse if it falls into the $234 \mathrm{~ms}$ window stretching from halfway towards the previous pulse and halfway towards the next pulse. Where a window includes more than one tap, the tap with the most accurate timing is kept and all other taps discarded. Out of a total of 314,671 taps, 10,768 such discards were made (3.4\% of the total), leaving 303,903 taps in a total of 641,775 pulse-level observations.

The dependent variable tap_num is the number of times a participant tapped each of a rhythm's $N$ different pulses during each 30-second performance. For example, in any performance of the rhythm (2 $\left.2 \begin{array}{llll}2 & 1 & 2 & 1\end{array}\right)$, which can also be written as an indicator vector $\left(\begin{array}{lll}1 & 0 & 1\end{array}\right.$ $\left.0 \begin{array}{llll}0 & 1 & 0 & 1\end{array}\right)$, each of the 8 pulses (each lasting $234 \mathrm{~ms}$ ) will be repeated 16 (or 17) times and there may be anything from 0 to 16 (or 17) taps within the window centred on that pulse over its 16 (or 17) repetitions. Hence this is a binomial-like variable (it is theoretically possible for each pulse to be separately modelled as a Bernoulli variable, which would have allowed for learning to be modelled within each performance, but this was not computationally feasible).

\subsubsection{Tap velocity}

The dependent variable tap_vel is the velocity of the tap at each pulse. Note that a velocity of 0 corresponds to no tap but, crucially, all such untapped observations were removed in order to allow for the effects on tap probability and tap velocity to be disambiguated. (A model using some form of zero-inflation would be an alternative way of disambiguating these two aspects but was not computationally feasible.) The MIDI value for velocity is a 7-bit integer, hence non-zero velocities are integers from 1 to 127 . The upper value is best considered as being censored because any tap harder than 127 MIDI units is still recorded as 127. A histogram of the tap velocities showed a substantial peak in the number of these maximum velocity values (see Figure 13). There were few values close to the minimum velocity of 1 MIDI unit. For these reasons, tap velocities were modelled as normally distributed (conditional on the predictors) with upper censoring. In the model, this variable was standardized (divided by its standard deviation) but not mean-centred.

The process used for variable selection (detailed in Sec. 2.3.2) is computationally intensive so, for this purpose only, tap_vel was averaged over each periodic pulse in each performance and modelled as normally distributed (conditional on the predictors), without censoring. 


\subsubsection{Tap timing error}

The dependent variable tap_delta is the signed time difference between each tap and its closest pulse (regardless of whether the pulse is cued or uncued). The range of values in milliseconds (i.e., prior to standardization) is, therefore, $[-117,117]$. Both boundaries are truncated because values outside this range are not recorded in the data set: as detailed in Section 2.1.2, they are automatically assigned to the previous or following pulse or removed. For this reason, tap timing was modelled as normally distributed (conditional on the predictors) but truncated above and below (a truncated student- $t$ distribution was also tried but performed less well under cross-validation). In the model, this variable was standardized (divided by its standard deviation) but not mean-centred. Unlike the tapping accuracy $\left(t a p \_a c c\right)$ variable, the tap-timing error is signed (hence sensitive to whether taps are late or early) and, because the timing error is measured with respect to the closest pulse - cued or uncued - it does not penalize incorrect taps. Also tap_acc refers to an entire performance, whereas tap_delta refers to each tap.

As with tap_vel, for variable selection only, tap_delta was averaged over each periodic pulse in each performance and modelled as normally distributed (conditional on the predictors), without truncation.

\subsection{Predictors}

For a rhythm's timing pattern to be reproduced with perfect accuracy, its IOI sequence (or a lossless encoding thereof, such as the Fourier transform of the IOI sequence, the rhythm's indicator vector, or the Fourier transform of the indicator vector (Milne \& Herff, 2020), or metricization such as detailed by Povel and Essens (1985) must be in memory and able to be retrieved sufficiently fast. For extended non-isochronous rhythms, such as those used in this experiment, demands on working memory may be challenging. As suggested by Povel, one cognitive mechanism is to encode the rhythm by chunking familiar elements spaced by an isochronous metrical grid (an underlying "beat"). For example, the pentatonic rhythm (1 01

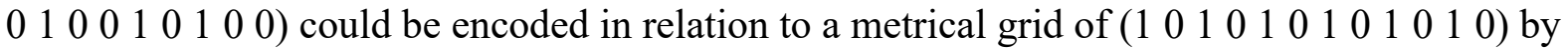
chunking just three familiar elements - "no" tap, tap "on" the grid, and tap halfway "off" the grid. This leads to the following representation of the pentatonic rhythm: "on", "on", "on", "off", "off", "no". Arguably, this may reduce demands on working memory, whilst still keeping timing accurate; and, so long as the rhythm can be chunked from elements familiar to the performer, it could be an effective mechanism. However, for sufficiently complex

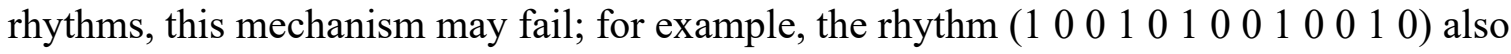
has five onsets but, due to them being arranged across 13 pulses instead of 12 , any "chunking" of them into the elements "on", "off", and "no" must stretch over two periods of the rhythm (because 13 is a prime number, whereas 26 is divisible by 2). This leads to the substantially longer (hard-to-remember) chunking sequence "on", "off”, “off”, "no", "on", “off”, “off”, “no”, “on”, “on”, “off”, “no”, “on”.

In the event of failures of metricized chunking or other lossless encodings, might we see evidence of alternative lossy encodings that influence the probability or velocity of taps at each pulse in the rhythm? Many of the pulse-level predictors (whose values vary by pulse) introduced below can be thought of as lossy, sometimes crude, summaries of cue locations in rhythms that may play a role in increasing the probability of tapping (and not tapping) correctly; as subsequently detailed in the description of the pulse-level predictorproj_cent, an obvious example is the region close to the circular mean of the rhythm which, in the absence of any other information, represents a sensible place to tap. (A natural representation of a periodic rhythm is a set of points on a unit circle where the angle of each point represents its onset time modulo the period. For any set of points arranged on a unit circle, their circular or directional mean is the direction of those points' centroid; their circular variance is 1 minus 
the centroid's distance from the circle's centre and is, therefore, a value between 0 and 1 (Mardia, 1972).) The rhythm-level predictors (whose values vary by rhythm but are constant for every pulse in any given rhythm) operate at a slightly higher level in that they are related to the extent to which rhythmic features can be learned, given cognitive constraints on memory capacity, speed of processing and retrieval, and distinguishability of structural features of the rhythm. They can also characterize the extent to which any given type of lossy encoding can accurately represent a rhythm. There are also two predictors which operate at the performance level; that is, they change only between performances.

All these predictors are now summarized; unless otherwise noted, all predictors were hypothesized prior to undertaking the experiment. In each case, we identify the hypothesized effect of each predictor on tapping behaviour, and the cognitive mechanisms (lossy encodings and resulting mechanisms) we hypothesize they indicate. Unless the mathematical specification is very simple, the predictors are described verbally in this section; formal mathematical definitions of the more complex predictors are provided in Appendix A. 2.

\subsubsection{Performance-level predictors}

- $\quad$ erf_num: for each performance, the number of completed performances of the rhythms by the participant at the time of that trial; so this is a number between 1 and 44 or 46 (depending on which group of rhythms the participant was randomly assigned to). We expect this to be positively associated with tapping accuracy due to participants becoming more familiar with task and having had the opportunity to develop or select useful strategies. Hence this predictor is indicative of learning.

- repetition: for each performance, the number of completed performances of the rhythm just performed at the time of that trial. Most rhythms were played twice by each participant, but there were a few instances of a participant playing the same rhythms 3 times; hence this predictor has values 1,2 , or 3 . We expect this to be positively associated with tapping accuracy due to participants having gained greater familiarity with each specific rhythm with each successive performance of it. Hence this predictor is indicative of learning.

\subsubsection{Rhythm-level predictors}

- $N$ : the number of pulses in the rhythmic loop. We expect rhythms with higher $N$ to be less accurately tapped because the duration of the minimal sequence of interonset intervals that needs to be (serially) recalled is longer; furthermore, they repeat fewer times over each 30-second performance, which reduces the opportunity for learning the pattern. Hence this predictor is indicative of the influence of short-term memory capacity and decay.

- $K$ : the number of cues in the loops. We expect rhythms with higher $K$ to be tapped less accurately because the length (or information content) of the minimal sequence of interonset intervals that needs to be (serially) recalled is greater. Hence this predictor is indicative of the influence of short-term memory capacity.

- mean_IOI: the mean IOI between cues, which is given by $N / K$. This predictor was thought of after the initial data analysis, when it became apparent that it might play a useful predictive role. We initially hypothesized it to be positively associated with tapping accuracy because the speed of information flow (the IOI sequence) in the rhythm is slower, in which case this predictor is indicative of cognitive constraints on processing speed. However, after the full modelling had been completed, it became apparent that this predictor was indicating a quite different cognitive mechanism: rhythms with low mean_IOI have events occurring more frequently (across time, they have high cue 
density) and so encourage tapping regardless of its correctness; in this case, this predictor is indicative of a simple mechanism derived from a very reductive lossy compression of the rhythm (its cue density).

- evenness: evenness quantifies the extent to which interonset intervals are of a similar size (Amiot, 2009; Milne et al., 2017; Milne et al., 2015). See Appendix A. 2 for a formal mathematical specification. Rhythms with low evenness will, therefore, tend to have groups (cues clustered in time) and gaps (uncued pulses clustered in time). For example, consider the rhythms $\left(\begin{array}{lll}1 & 1 & 0\end{array}\right)$ and $\left(\begin{array}{llll}1 & 1 & 0 & 0\end{array}\right)$ with respective evennesses of 0.866 and 0.707 ; the latter is more uneven than the former and the contrast between the short and long IOIs are greater. As such, more uneven rhythms have more discriminable asymmetries, which can facilitate position-finding - the ability to estimate where one is within the rhythm. The concept of position finding is more commonly applied to musical scales (Balzano, 1982; Browne, 1981) where it has been recently demonstrated that asymmetries facilitate position-finding (Pelofi \& Farbood, 2021). For these reasons, we expect rhythms with higher evenness to be harder to tap. (It is interesting to note that if the task does not require position-finding, symmetries can be beneficial because working memory can make use of redundancies to reduce information content (Attneave, 1955). This predictor is, therefore, indicative of the extent to which tapping behaviour is influenced by temporal position-finding.

- IOI_ent: interonset interval entropy quantifies the unpredictability of the distribution of

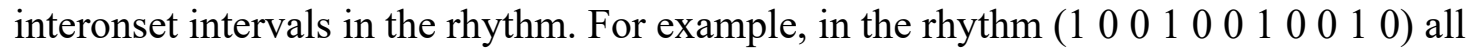
but one IOI is of size 3 pulses, the entropy is lower and a performer can be fairly confident that a guess of 3 will be correct; conversely, in $\left(\begin{array}{lllll}1 & 0 & 0 & 1 & 0\end{array}\right)$ half the IOIs are of length 3 and half of length 2, so a guess of length 3 is less likely to be correct. Hence, we expect rhythms with lower interonset interval entropy to be easier to tap. This predictor is, therefore, indicative of a mechanism derived from a lossy compression of the rhythm (the distribution of IOIs between consecutive cues, ignoring their order).

- int_ent: interval entropy is similar to IOI entropy but considers the distribution of all temporal intervals between cues in the rhythm, modulo the period - not just those between consecutive cues. For example, in the rhythm (1 $\left.\begin{array}{lllllllll}0 & 0 & 1 & 0 & 0 & 1 & 0 & 0 & 1\end{array}\right)$ there is one IOI of 2 pulses (between consecutive cues), three IOIs of 3 pulses (between consecutive cues), two IOI of 5 pulses (between non-consecutive cues), two of 6 pulses (between nonconsecutive cues), three of 8 pulses (between non-consecutive cues), one of 9 pulses (between non-consecutive cues). Hence, in this rhythm, it would be a good idea tap 8 pulses after a cue rather than 9. For each rhythm, therefore, this predictor quantifies the utility of this mechanism by specifying the unpredictability of the distribution of all interonset intervals in the rhythmic cycle, not just those between consecutive cues as in IOI_ent. For the same reasons as with IOI entropy, we expect rhythms with lower interval entropy to be easier to tap. Like IOI_ent, this predictor is, therefore, indicative of a mechanism derived from a lossy compression of the rhythm (the distribution of IOIs between all cues up to the period, ignoring their order).

- CQ: the coherence quotient, developed by Carey $(2002,2007)$ for musical scales, quantifies the extent to which knowledge of the generic distance between any two cues provides information about their interonset intervals (the generic distance between two cues is the number of cues between them plus one). A mathematical definition of the coherence quotient is provided in the Appendix; here, we explain by example. Consider

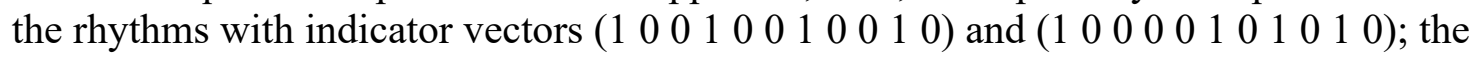
first rhythm has a higher coherence quotient (1) than the latter (0.4286). In the former, if we start at any cue and move to the next cue (move by one generic step), the interonset interval is either 2 or 3 ; if we start at any cue and move to the cue after next (move by two 
generic steps), the inter-cue interval is either 5 or 6 ; if we move by three generic steps, the inter-cue interval is either 8 or 9 . This rhythm has a maximal coherence quotient because there is no example where the interonset interval of a given number of generic steps is greater than the interonset interval between a greater number of generic steps. The second rhythm provides some counterexamples: if we start at any cue and move one generic step the interonset interval is either 2 or 5; if we move two generic steps, the inter-cue interval is either 4 or 7; if we move three generic steps, the inter-cue interval is either 6 or 9 . It is possible, therefore, for the inter-cue interval for a given number of generic steps to be greater than the inter-cue interval for a larger number of generic steps. Each such case is termed a coherence failure, and the coherence quotient divides the number of these coherence failures by the maximum possible number of coherence failures for a rhythm with $K$ onsets and subtracts from this from 1 . Hence, a maximally coherent pattern has a CQ of 1, a maximally incoherent pattern has a CQ of 0 . In this way, we can see how simply counting the cues (and not the pulses) can - depending on the rhythmic structure give greater or lesser information about their associated inter-cue intervals. For high coherence rhythms, the generic distance between pairs of cues is a lossy compression of their interonset interval: they are compressed because there are fewer cues than pulses in any nontrivial rhythmic period; they are lossy because they reduce the range of possible lengths for each IOI but do not precisely code its length. All well-formed rhythms whose long:short IOI ratio is less than 2 are maximally coherent, as are all WF rhythms with only one small IOI. This predictor, therefore, is indicative of a mechanism derived from a lossy compression of the rhythm (knowledge of $\left(\begin{array}{c}K \\ 2\end{array}\right)$ generic intervals gives information about $\left(\begin{array}{c}N \\ 2\end{array}\right)$ interonset intervals).

- SQ: the sameness quotient (Carey, 2002, 2007) is also derived from a consideration of the relationship between the generic distance between cues and their possible interonset intervals. In a rhythm with a high sameness quotient every generic interval typically has fewer different interonset intervals. In this sense, it is conceptually related to interval entropy but it is simpler in that it only counts the number of different such intervals rather than their distribution. Furthermore, as with $C Q$, it is normalized by dividing the number of different intervals for every generic interval by the maximum possible such number for a rhythm with $K$ onsets and subtracts from this from 1 . Because all non-degenerate WF rhythms have two interonset intervals per generic interval (all non-degenerate WF scales have Myhill's property (Carey \& Clampitt, 1989)) this means that, for our rhythms, this predictor is more highly correlated with $K(0.80)$ than it is with IOI_ent $(-0.71)$. This predictor is, therefore, indicative of a mechanism derived from a lossy compression of the rhythm (a crude estimate of the distribution of IOIs between consecutive cues, ignoring their order).

- balance: for a rhythm represented as weighted points on a circle, this is the circular (directional) variance of the rhythm (Milne et al., 2017; Milne et al., 2015). The more unbalanced a rhythm, the more informative of the cues' locations is the cues' circular mean; hence, the lower the balance, the better the cues' mean serves as a good lossy descriptor of the rhythm. Put differently, if the circular mean has been found, and the balance is low, tapping in the vicinity of that circular mean has a better chance of being correct than tapping in the other half of the rhythm's cycle. Furthermore, unbalanced rhythms are more distinctly asymmetric with respect to rotation, which may facilitate position-finding. This predictor is, therefore, indicative of a mechanism derived from the lossy compression of a rhythm using its circular mean and variance and, furthermore, of the extent to which tapping behaviour is influenced by temporal position-finding (like evenness). 
- duple_triple: this predictor is a simple way of quantifying how well a rhythm can be metrically coded; that is, chunked into familiar elements spaced by an isochronous beat (as detailed at the start of this section). Isochronous rhythms without acoustic accents are subjectively metricized into twos or threes (Bolton, 1894), so this predictor simply codes whether or not $N$ is divisible by either 2 or 3 . Note that any rhythm with $N$ that cannot be divided by 2 and cannot be divided by 3 , could be reinterpreted as a syncopated rhythm of length $2 N$ (or even $3 N$ ), which would mean this double-length rhythm can now be partitioned into pulses grouped into twos (or threes). But, clearly, this doubling (or tripling) in length represents an increase in the cognitive demands required (for example, both $N$ and $K$ would need to be doubled or tripled). This predictor is, therefore, indicative of the extent to which tapping is influenced by lossless metrical coding, which facilitates chunking to increase short-term memory capacity; it is also indicative of rhythmic familiarity given the prevalence of duple and triple times in Western music.

\subsubsection{Periodic pulse-level predictors}

The above predictors produce a single value for all pulses in any given rhythm. However, an arguably more interesting question is - given a set of rhythmic cues - what determines precisely which pulses in a given rhythmical structure are more likely to be tapped, tapped with greater velocity, or tapped early or late. To model such phenomena, we require predictors that are vector-valued functions of each rhythm's indicator vector; that is, if the rhythm has $N$ periodic pulses, the predictor has a value for every one of those $N$ pulses. The simplest, and probably most important, such function is the identity function (here denoted cue), which is the rhythm's indicator vector whose entries mark the presence or absence (with a 1 or a 0 , respectively) of a cue at each periodic pulse.

Given that the pulse-level predictors are calculated from a full period of the rhythm, they cannot influence tapping until at least one cycle of the rhythm has been heard. Furthermore, the accuracy with which a participant can internally estimate these features is likely dependent on how many cycles of the rhythm they have heard. Because it was not computationally feasible, we do not model trends over each performance, so any such learning effect is glossed over in our analysis (if interested in learning effects for these rhythms, these are analysed in Dean et al. (in press)). Over a 30-second performance, shorter rhythms repeat more often than longer rhythms; hence participants may more accurately estimate these features for the shorter rhythms. In our models, the effect of repetition is approximately accounted for by the rhythm-level predictor $N$ (the number of pulses in the rhythmic period), which was discussed earlier.

As detailed in Section 2.3.1, in our pulse-level models, we interact all other pulse-level predictors with cue. This allows us to assess how each predictor is associated with correct (cued) taps and incorrect (uncued) taps. When estimating tap probabilities, this means we can also assess how each predictor is associated with the discriminability of cued and uncued pulse. In signal detection theory, "discriminability" ( $d$-prime) is the difference between the probit-transformed probabilities of a "hit" or "true positive" (here, a cued tap) and a "false alarm" or "false positive" (here, an uncued tap). For example, consider a simple model where a predictor $x$ interacts with cue so that $y$ is predicted by $\beta_{0}+\beta_{1}$ cue $+\beta_{2} x+\beta_{3}($ cue $\times x)+$ $\epsilon$. At an uncued pulse, cue $=0$; at a cued pulse, cue $=1$. Hence, the effect of $x$ on uncued $y$ is given by $\beta_{2}$; the effect of $x$ on cued $y$ is given by $\beta_{2}+\beta_{3}$; and the difference between the cued and uncued effects of $x$ is given by $\beta_{3}$. (See Appendix A. 3 for a more in-depth explanation and an overview of the relationship to signal detection theory.)

One consequence of this modelling strategy is that any pulse-level predictor must have some variance for uncued as well as cued pulses; in the absence of this, the model's design matrix will be rank deficient. For example, Povel predicts that cues that are the last in any 
group, or the first in any group with three or more cues, have greater metrical accent; but this predictor says nothing about metrical accents at uncued pulses. For this reason, we do not include this as a predictor in our default models; instead, we substitute an alternative edgedetection predictor ( $e d g e$, which is detailed in the list below), which also makes predictions for uncued pulses (furthermore, we run an additional model containing the Povel predictors on only the cued pulses, thereby removing the problematic interaction).

Our pulse-level predictors are now outlined; they are mathematically specified in Appendix A. 2. Unless otherwise stated, these are novel predictors in a rhythmic context.

- cue: a binary indicator of whether or not a cue is present at that periodic pulse. Clearly, this variable represents an idealized performance; so, the higher its positive effect, the more cued pulses are treated differently to uncued pulses (e.g., tapping better discriminates between them). As outlined above, this predictor allows us to estimate how well participants can discriminate between cued and uncued pulses and how these influence tap velocity and timing.

- $A P M$ : this predictor is a way of quantifying metrical accents arising from isochronous beats induced by rhythmic cues. It is the column-sum of the circular autocorrelation phase matrix (it sums over all $N$ lags to leave only the $N$ phases). It is closely based on Parncutt's pulse-match salience (1994) and Eck's (non-circular) autocorrelation phase matrix (2006). (Parncutt's summation limits are mathematically consistent with his description, but not optimal for our purposes.) In summary, a pulse gets a higher $A P M$ value the greater the number of cues meeting two conditions: the cue has a distance of $n$ pulses from the pulse of interest and that cue is separated by $n$ pulses from any other cue modulo the period. We would expect pulses with higher $A P M$ values to be more likely tapped, tapped harder, and possibly tapped earlier because they are predicted. Given metrical coding (as indicated by duple_triple), this predictor is indicative of the use of metricization and the chunking this facilitates (where metrical weights arise from possibly gapped sequences of cues IOIs).

- seq_exp: sequential expectation is a different way of quantifying metrical accents arising from isochronous beats induced by rhythmic cues. It differs from $A P M$ in the precise mechanism that implies isochronous beats: $A P M$ is concerned with the number of rhythmic cues coinciding with every possible beat; seq_exp is concerned with the lengths of preceding sequences of cues coinciding with every possible beat. For all sequences of isochronous cues, the predictor assigns a higher expectation of a cue occurring on the pulse that continues that isochronous sequence. However, that expectation is weighted according to the size of the interonset interval in that prior sequence of regular cues, and that regular sequence's length. For example, consider the periodic rhythm (1 $\left.\begin{array}{llllll}1 & 0 & 1 & 1 & 1 & 0\end{array}\right)$; the first pulse is a continuation of an infinite sequence of cues with interonset intervals of 2 pulses; it is also a continuation of an infinite sequence of cues with interonset intervals of 3 pulses (the former adds more to the predictor's value because the IOI of the cue sequence is smaller than the latter's); both of these imply this pulse gets a high seq_exp value. The fifth pulse is a continuation of a sequence of one IOI of 1 pulse, and a continuation of an infinite sequence of IOIs of 2 pulses (the former contribution is reduced because it is a short - shortest possible - sequence, even though its IOIs are of the smallest possible size). The second (uncued) pulse does not continue any sequences, so would get a value of zero; the final (uncued) pulse continues a sequence of two IOIs of 1 pulse, so is greater than zero. The weightings, as a function of IOI size and sequence length were separately optimized and are detailed in the Appendix A. 2. As with APM, we would expect pulses with higher seq_exp values to be more likely tapped, tapped harder, possibly tapped earlier. Given metrical coding (as indicated by duple_triple), 
this predictor is indicative of the use of metricization and the chunking this facilitates (where metrical strength is derived from unbroken sequences of cue IOIs).

- edge: this predictor is concerned with the grouping structure of the rhythm; it finds the edges of groups (clusters of cues) and the edges of gaps (clusters of uncued pulses) by adapting standard edge-detection techniques for images (convolving with the derivative of a Gaussian kernel). In vision, edge detection is an important means of reducing information whilst preserving the overall the structure of an image. It is possible encoding just the temporal edges in rhythms may hold similar benefits. Furthermore, this technique is one way to generalize Povel's accentual weights (which are high at the starts and ends of cue clusters) to also include uncued pulses; for example, the bold entries in each of these rhythms show where edges are detected (i.e., the value of $e d g e$ is high):

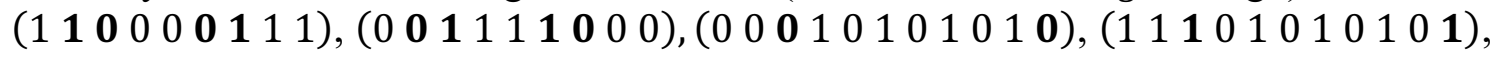

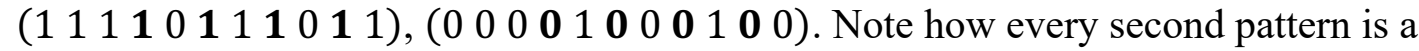
"flipped" version of the previous pattern (cues are replaced by silences, and vice versa), but this has no effect on the detected edge locations. Furthermore, with clusters comprising just two cues, we typically find the second cue to receive a higher edge value; for example, in the pattern $\left(\begin{array}{lllll}0 & 0 & 1 & 1 & 0\end{array}\right)$, the respective edge values are $(0.00,0.22,0.15$, $0.27,0.20,0.00)$; it is interesting to note that the greater weight on the second cue of a 2cue cluster corresponds with Povel's suggested accent. Following Povel's theory and perceptual experiments on accenting (Povel \& Essens, 1985), we would expect highedge pulses to be tapped with greater probability and with greater velocity (for reasons detailed later, we find edge mostly has the opposite effect). From a cognitive perspective, this predictor is indicative of mechanisms derived from a lossy compression of the rhythm (the locations of its edges).

- height: this predictor is also concerned with the grouping structure of the rhythm: pulses closer to the starts of cue clusters have low height; pulses closer to the ends of cue clusters have high height. The height of each periodic pulse is the sum of the distances between that pulse and all cues following it (up to one period after) minus the sum of the distances between that pulse and all cues preceding it (up to one period before); hence, its units are pulses. The term "height" arises from its meaning when applied to pitches in a musical scale: the height of any pitch is the average pitch height of the scale relative to that pitch. These values are normalized to the size of the period by dividing by $N$ resulting in semitone units (or, in a rhythmic context, pulse units). This predictor is indicative of biases towards tapping behaviour at the starts or ends of groups, some of which may result from motoric considerations or from biases towards remembering the lengths of gaps compared to remembering the lengths of clusters: (a) taps later in a sequence of fast taps may become weaker due to fatigue (Repp et al., 2005); (b) cues at the starts of clusters may be harder to predict than those towards the end; (c) cues at the ends of groups may be tapped earlier than those at the start (Repp, 2003; Snyder et al., 2006). In combination with edge, this allows for a variety of different "contours" to be quantified over clusters.

- proj_cent: the projected centroid is a pulse-level generalization of the rhythm-level balance predictor and represents how a simple statistical summary of a rhythm (its centroid) may influence predictive behaviour. Consider cued and uncued pulses as points on a circle, where angle denotes their time of occurrence. For any given pulse, proj_cent is the signed magnitude of the rhythm's centroid when projected onto the diameter passing through that pulse. This implies that pulses close to the circular mean of the cues have greater proj_cent than pulses distant from the circular mean; the more unbalanced the rhythm (the lower its circular standard deviation) the greater the variation in these values. Its values are in the interval $[-1,1]$. Figure 1 illustrates how proj_cent is 
determined for two rhythms with differing balance levels. This predictor is indicative of a mechanism derived from the lossy compression of a rhythm using its centroid (i.e., its circular mean and its variance).
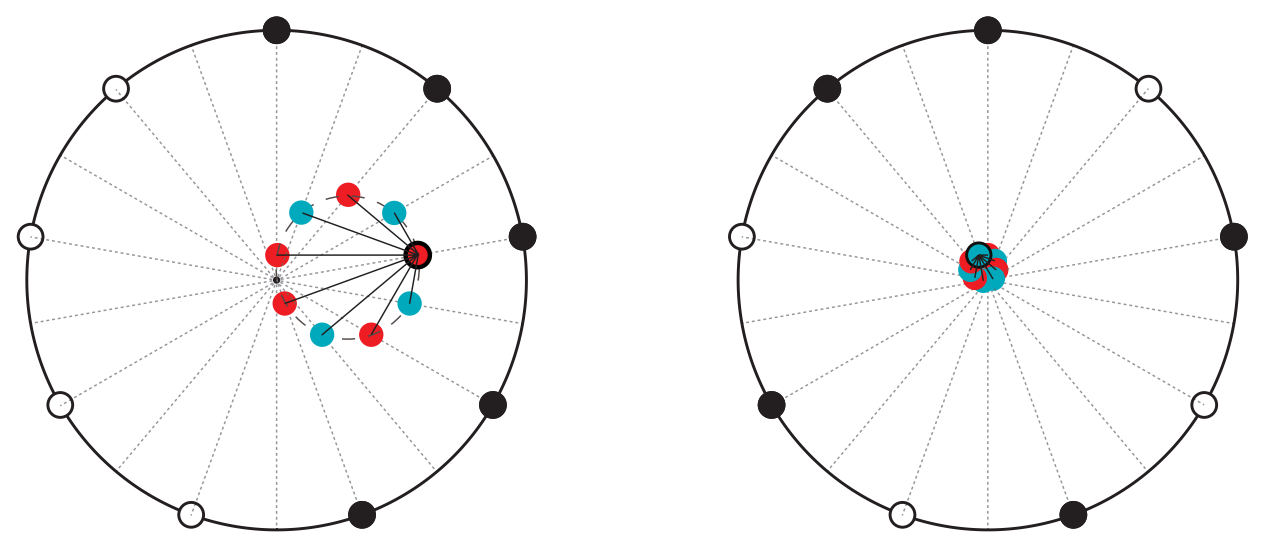

Figure 1. Two rhythms illustrated in circular format: cued pulses are depicted with black disks; uncued pulses with white disks. The first has lower balance than the second. A diameter (dashed) passes through every pulse in the rhythm and perpendicular projection lines (solid) are drawn from the rhythm's centroid (coloured disk with black outline) until it intersects with each pulse's diameter. Each intersection is depicted with a coloured disk centred on it. The distance of any intersection from the circle's centre gives the magnitude of proj_cent for that pulse; if the line meets the diameter on the side of the circle opposite to the pulse, it is negative (positive values are coloured red; negative are coloured blue). Note how the range of proj_cent values is greater in the first low-balance rhythm than in the second high-balance rhythm; in a perfectly balanced rhythm (one whose centroid is at the circle's centre), every pulse has a proj_pred value of 0 . For those familiar with audio recording, this is analogous to a figure- 8 microphone polar pattern: the sound intensity produced is affected by its direction (pulse position) and the overall gain (imbalance); the sound's phase is reversed by a 180 degree rotation.

- Markov2, 3, 4, ...: The predictor Markov2 represents the optimal 2-step Markov predictor for the cue sequence. That is, at each pulse, we consider the length-2 binary sequence of cues or non-cues over the previous two pulses. We then consider all occurrences of that cue sequence in the rhythm, and calculate the proportion that are

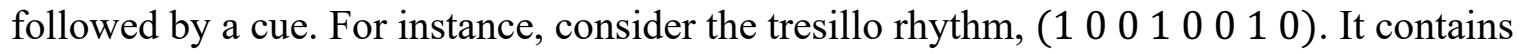
three distinct length-2 sequences: $\left(\begin{array}{ll}0 & 0\end{array}\right),\left(\begin{array}{ll}0 & 1\end{array}\right)$ and $\left(\begin{array}{ll}1 & 0\end{array}\right)$. In this rhythm, the sequence $\left(\begin{array}{ll}0 & 0\end{array}\right)$ is always followed by 1 , and the sequence $\left(\begin{array}{ll}0 & 1\end{array}\right)$ is always followed by 0 , so the Markov predictor attaches probabilities of 1 and 0 to those following pulses, respectively. On the other hand, the sequence $\left(\begin{array}{ll}1 & 0\end{array}\right)$ occurs three times, followed by a 0 twice and a 1 once, so the optimal prediction after $(10)$ would be a cue probability of $1 / 3$. Altogether, therefore, the Markov 2 cue probabilities for the tresillo rhythm are $(1 / 3,0,1 / 3,1,0,1$ / $3,1,0)$. The predictors Markov3 and Markov4 are defined similarly for the optimal order-3 and order-4 Markov predictors. For our data, Markov2, Markov3, and Markov4 are highly correlated so it is not useful to include more than one in the same regression. Of the three predictors, Markov2 resulted in the model with the highest crossvalidated likelihood; henceforth, we consider only Markov2. Our focus in this paper is on the structural features of rhythms, so we did not attempt Markov predictors that track probabilities over the course of the experiment, nor over a corpus of culturally familiar music (such as can be modelled within the IDyOM framework, Pearce, 2005, 2018). This predictor was introduced after the experiment had been performed. It is a probability, hence lies in the interval $[0,1]$. It is indicative of a mechanism derived from a lossy compression of a full rhythm (only the previous 2,3 , or 4 IOIs rather than all $K$ ). 
- tap_lag1: For the tap velocity and timing models, this is a binary indicator for whether the previous pulse was tapped. For computational reasons (see Sec. 2.1.2), in the tap probability model, the data for each pulse (modulo the period) was aggregated over all its repetitions in each 30-second performance. So, for this model, this predictor is the proportion of such taps occurring over each performance. Although not of primary interest in this investigation, it is included to help soak up residual autocorrelation and to allow the model to partially control for how past tapping behaviour influences current tapping behaviour.

\subsection{Modelling approach}

Here, we provide an overview of the modelling strategy used in this paper. Other aspects of this experiment and ways of analysing these data will be addressed in associated articles: time-series analyses of their temporal features (Dean et al., in press); point process analyses; liking ratings.

\subsubsection{Bayesian multilevel regression}

We used Bayesian multilevel (mixed effects) regression including as many random effects as computationally feasible (bear in mind that the dataset is very large - 651725 observations and most of the models took many days to complete fitting). Maximal random effects structures are generally recommended to ensure uncertainties of estimated effect sizes are not underestimated (Barr et al., 2013). In the first performance-level tapping accuracy model, all predictors' effects were allowed to vary by participant and by rhythm as random effects, hence the random effects' structure was maximal. In the other models, it was unfeasible to include this many random effects due to the required runtime for the models (they would have taken multiple weeks to fit). But it was possible to include the intercept and cue (the most important predictor) as random effects varying by participant and rhythm; that is, in their R-style formulas, these models included the terms $\cdots+(1+$ cue $\mid$ participant $)+(1+$ cue |rhythm); the complete formulas for each model are provided in Tables 3, 4, 6, and 7. Note that - as reported later - the resulting uncertainties of the effects are dwarfed by their size, so the lack of a maximal random effects structure is unlikely to make any substantive difference to our inferences.

The regressions were performed using the brms package (Bürkner, 2017, 2018), which is a front-end for the Bayesian inference and MCMC sampler Stan (Carpenter et al., 2017). This means we can estimate the entire posterior probability distribution of each coefficient, so we do not report $p$-values. Instead, we report the evidence ratios and posterior probabilities that each coefficient, or sums of coefficients that define a contrast of interest, is greater than (or less than) zero. We interpret evidence ratios greater than 19 as strong evidence in favour of a directional hypothesis (this is loosely analogous to a one-sided $p$-value of 0.05 (Makowski et al., 2019)). All discrete regression inputs (cue and, in the tap velocity and timing models, tap_lag1) were dummy (binary) coded; all continuous regression inputs were standardized to have a mean of 0 and a standard deviation of 1 with the exception of tap_lag 1 in the tap probability model, which was kept as a proportion between 0 and 1 to keep it on the same scale as in the tap velocity and timing models. All effects were given a prior with a Student's $t$-distribution having a mean of 0,3 degrees of freedom, and a scale of 1 . For standardized regression inputs, this is a weakly informative prior which, centred at zero, helps to regularize the effect sizes towards plausibly small to medium values. For each model, as many grouplevel effects were included as possible whilst keeping the fitting of the model computationally feasible. The full model specifications are provided in Tables 3, 4, 6, and 7 . Further information on how to interpret the coefficients of the models, given their distribution and link function, is provided in Appendix A. 3. 
All reported models fitted without divergences, had $\hat{R}$ values below 1.05, and had at least 400 effective samples per effect. Posterior predictive checks confirmed that appropriate distributional families had been chosen.

\subsubsection{Variable selection}

For our rhythms, there are some extremely high multicollinearities between some of the predictors, which means there are considerable redundancies in the information they provide. Given that each predictor is individually meaningful, a PCA type approach - where the resulting subset of predictors lose their simple interpretation - is not desirable. In order to select a good subset of predictors, we use the technique of projection predictive variable selection as implemented in the R package projpred (Piironen et al., 2020; Piironen \& Vehtari, 2017). This technique is related to the familiar forward stepwise selection but is protected from overfitting and double-use of the data by testing each model, with crossvalidation, against a fitted reference model that contains all the predictors from which the subset is chosen. This procedure returns a sequence of predictors, ordered by decreasing predictive importance, and these can be successively added in order to improve the projected fit. We added predictors in this order, but any predictor that introduced a VIF greater than 10 - for itself or any other predictor - was not added and we instead proceeded to the next predictor in the list. No previously added predictors were removed. This means that each final model contains a set of important predictors such that no predictor can be regressed on the remaining predictors with an $R^{2}$ greater than 0.9 .

\section{$3 \quad$ Methods}

\subsection{Participants}

One hundred and eleven first-year psychology university students recruited from Western Sydney University participated in the experiment. One participant did not provide demographic information; the following summaries are for the remaining 110 participants. There were 89 female and 21 male participants, their mean age was 22.4 (in the range 18 to 48 , with a standard deviation of 6.2). Their mean Ollen musical sophistication index (Ollen, 2006) was 86 (in the range 10.5 to 873 , with a standard deviation of 113.3 , with 2 participants scoring higher than 500). (The value of 500 is considered the transition between lower and higher sophistication). Their mean number of years of musical practice was 0.8 (in the range 0 to 13 , with 85 participants having had no practice, and 6 having had more than 5 years regular practice).

We did not collect data on the participants' cultural heritage or the types of music they listened to. However, in experiments conducted subsequently with students recruited from the same source we obtain the following statistics. Country where you grew up: Australia, New Zealand, or a European country (83\%); Middle East (3\%); South Asia (5\%); East Asia (3\%); Southeast Asia (5\%). The vast majority (98\%) listened predominantly to Western music genres such as pop, classical, jazz, and R\&B; only 10\% listen to any non-Western music genres, and only $5 \%$ to genres with a possibility of having distinctly non-Western rhythms (Arabic, Chinese classical, Greek). So, in terms of cultural heritage, this cohort has a reasonable amount of diversity but, in terms of musical exposure, they are relatively homogenous and strongly focussed on music with the duple and triple metrical hierarchies conventionally used in Western music.

\subsection{Materials}

Participants tapped on a Roland Handsonic HPD20 (the Handsonic has multiple adjacent pads and participants were told they could tap on whichever pad was most comfortable for them). Each rhythm played for 30 seconds (129 pulses). The rhythmic loops (as exemplified 
in Table 2) comprised isochronous pulses each sounded with a cymbal (IOI $234 \mathrm{ms,}$ $256 \mathrm{bpm}$ ), and cues sounded with a piano tone (middle C) on some pulses. The audio from the cues was subject to the same audio buffer latency (128 samples, $\sim 3 \mathrm{~ms})$ as was the audio coming from the tap: both MIDI streams were routed via Max 7 (Cycling '74, 2021) into a standalone Pianoteq 5 (Modartt, 2021), which is the synthesizer producing the piano sounds.

The 91 rhythms - patterns of cues - are all possible non-degenerate well-formed rhythms with between two and eleven cues that are subsets of 3 to 13 pulses and have no interonset intervals larger than 5 pulses; they are listed in Appendix A. 4. Each tap produced an additional piano tone a perfect fourth above the cue (the $\mathrm{F}$ above middle $\mathrm{C}$ ). The pulse IOI is in the range of typical tempos in pop music and corresponds to a quaver (1/8th note) at a tempo of approximately 128 beats per minute (as would often be sounded by a high-hat pulse). This also means that cues on consecutive pulses are within Povel's $250 \mathrm{~ms}$ grouping limit, while all other pairs of cues are substantially outside it. Pulses were sounded to make the often very difficult tapping task a little easier - sounded pulses offer the "subdivision benefit" (Repp \& Su, 2013), which aid predicting the timing of cues comprising several pulses. Each rhythm was started at a random phase; this was done in order to allow the effect of phase to be randomized out when aggregated across observations (as in Parncutt (1994)); this is because non-structural features such as these are not our focus. Examples of three different well-formed rhythmic loops are shown in Table 2.

Table 2. Three well-formed rhythms. The first rhythm has 8 pulses, 5 cues $(N=8, K=5)$, and indicator vector

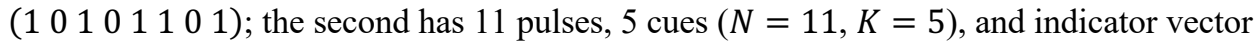

(1 00010100110001 ); the third has 12 pulses, 7 cues $(N=12, K=7)$, and indicator vector

(1 0100100110010 1). Because they are well-formed, the long and short IOIs are distributed as evenly as possible. Every pulse in every rhythm is uniquely identified by the number $n$, which counts upwards from 0 to $N-1$, and 0 is aligned to the first cue when the rhythm is written such that the small IOIs are as close to the end as possible.

\begin{tabular}{|c|c|c|c|c|c|c|c|c|c|c|c|c|}
\hline$n$ & 0 & 1 & 2 & 3 & 4 & 5 & 6 & 7 & & & & \\
\hline Pulses (cymbal) & $x$ & $x$ & $x$ & $x$ & $x$ & $x$ & $x$ & $x$ & & & & \\
\hline Cues (piano) & $x$ & & $\times$ & & $\times$ & $\times$ & & $\times$ & & & & \\
\hline $\bar{n}$ & 0 & 1 & 2 & 3 & 4 & 5 & 6 & 7 & 8 & 9 & 10 & \\
\hline Pulses (cymbal) & $x$ & $x$ & $x$ & $x$ & $x$ & $x$ & $x$ & $x$ & $x$ & $x$ & $x$ & \\
\hline Cues (piano) & $x$ & & & $x$ & & & $x$ & $x$ & & & $x$ & \\
\hline $\bar{n}$ & 0 & 1 & 2 & 3 & 4 & 5 & 6 & 7 & 8 & 9 & 10 & 11 \\
\hline Pulses (cymbal) & $x$ & $x$ & $x$ & $x$ & $x$ & $x$ & $x$ & $x$ & $x$ & $x$ & $x$ & $x$ \\
\hline Cues (piano) & $x$ & & $x$ & & $x$ & & $x$ & $x$ & & $x$ & & $x$ \\
\hline
\end{tabular}

\subsection{Procedure}

The tapping task reported in this paper occurred in blocks 1, 3, 5, and 7 of an 8-block experiment. Participants listened to the rhythm and were encouraged to start tapping as accurately as possible as soon as they felt ready (their written instructions defined an "accurate performance" as "one where you tap only where a piano sound occurs, and your taps are closely synchronized with the piano". After each rhythm finished, participants gave a liking rating using a five-point scale (labelled "I liked it a lot" at the top, "I disliked it a lot" at the bottom, and "I feel neutral about it" in the middle); these data are not considered in this article. The 91 rhythms were divided into four disjoint sets of sizes 23, 23, 23, and 22, and each participant played every rhythm from one set in random order over two separate blocks (1 and 3). That participant then played the same set of rhythms again, also in two blocks (5 
and 7) and in random order. In a very few cases, due to procedural errors, a rhythm was tapped 1 or 3 times instead of the intended 2. Different tasks - data from which are not reported in this paper - were undertaken in the even-numbered blocks. In each of blocks 2 and 6 , participants adjusted four well-formed rhythms with a continuous slider that controlled the ratio between the long and short interonset intervals; they were asked to find the slider position that made the rhythm "sound as good as possible". In each of blocks 4 and 8, participants chose their favourite from four sets of rhythms, each set comprising between three and nine different but related rhythms, some well-formed, some not. The tasks were interleaved to provide sufficient variety to maintain participants' interest over the course of the experiment.

\section{$4 \quad$ Results and discussions by model}

\subsection{Rhythm-and pulse-level visualizations}

In this section, we provide some visualizations of the data; from these we make some preliminary and somewhat speculative observations, which are formally tested in the analytical models presented after this section. Figure 2 shows the average tapping accuracy (Sec. 2.1.1) for all 91 rhythms. These are the population means from a model where intercept and rhythm are group-level effects varying by participant; that is, the model has the following R-style formula: tap_acc $\sim 0+$ rhythm + (rhythm | participant $)$. On the left, the rhythms are ordered by $N$ (number of pulses in the rhythmic period) and, within each $N$, by $K$ (number of cues in each rhythmic period); on the right, they are ordered by $K$ and, within each $K$, by $N$. There are some obvious patterns, which are particularly noticeable in the lefthand plot: rhythms with higher $N$, and lower $K$ within each $N$ (higher mean interonset interval), seem harder to tap. There is possibly also an effect from whether or not the rhythm can be grouped into twos or threes.

Figures 3 and 4 summarize tapping behaviour at the pulse level - they show the smoothed distribution of tap velocities over each rhythm's period, aggregated over all performances (and participants). The smoothing was achieved by circularly convolving the data with a Gaussian kernel with a standard deviation of 10 milliseconds; the method is detailed in Appendix A. 5. The rhythms shown in Figures 3 and 4 are useful examples because they demonstrate the wide range of tapping behaviours arising from combinations of three different $N$ and three different $K$; analogous figures for every tested rhythm are available in Appendix A. 6.

Note that the shape of each plot arises from three possibly independent aspects of the data: the probability of a tap occurring at each periodic time location; the velocities of all occurring taps; the timings of all occurring taps. This means they give a good overview of the accuracy of the tapping and where consistent errors occur, both within and between rhythms.

It is apparent from these examples (which are not exceptional) that some rhythms are tapped with considerably more accuracy than others and that, within each rhythm, some cues are tapped with greater probability, velocity, or temporal accuracy than are other cues. Overall tap accuracy is more precisely quantified by the model in Section 4.2; the remaining three aspects are separated out in Section 4.3-4.5 which, respectively, report models of tap probabilities, velocities, and timing errors using rhythm-level and pulse-level predictors. Prior to reporting this modelling, it is useful to point out a few observations arising from these three figures:

1. Amongst the $N$ depicted in Figure 3,8 is tapped most accurately and 13 least accurately. But one of the rhythms in 13, which has a large number of cues, is tapped, perhaps surprisingly, rather well. 
2. Following on from the previous comment, rhythms with only a few cues - the left-hand column in Figure 3 - seem to be harder to tap accurately than those with many closely spaced cues. This is consistent with the observation from Figure 2.

3. In rhythms with clear groups (clusters of cues), the first cue is often the weakest and the medial cues can be stronger than the last. This can be seen in Figure 3, but is particularly clear in Figure 4, which shows three rhythms with obvious two-group structures. The models in Sections 4.3 and 4.4 help us to see whether this results from tap probabilities or from velocities of those taps.

The first of these observations is unsurprising: for our participants, 8 is a very commonly heard subdivision of the period (e.g., eighth notes in common time), while 13 is very uncommon; furthermore, 10 and 12 (the former also being unfamiliar) can be metrically subdivided into a regular beat each comprising two pulses, while 13 cannot. The second observation was not expected beforehand; it is discussed in more detail later. The third observation is surprising because prior experimental evidence has shown that the starts and ends of clusters (groups) of cues are perceptually and performatively accented (Povel \& Essens, 1985; Povel \& Okkerman, 1981; Repp et al., 2005). Furthermore, these results seem to contradict a widely held conception of how humans process rhythmical groups: "Cyclic permutations like $4122223,1222234,222234$ 1, and 223412 2, when presented repetitively (which is the case in all our experiments), are perceptually identical: on the basis of the 'gap' principle of Garner (1974), subjects will always hear the sequence as 122223 4, that is, ending with the largest interval." (Essens \& Povel, 1985, p. 419).

In order to determine more precisely which aspects of rhythmic structure are most relevant to which performance outcomes - overall accuracy, probability of tapping, velocity of taps, timing errors of taps - we now model each of these outcomes with the predictors detailed in Section 2.2. 

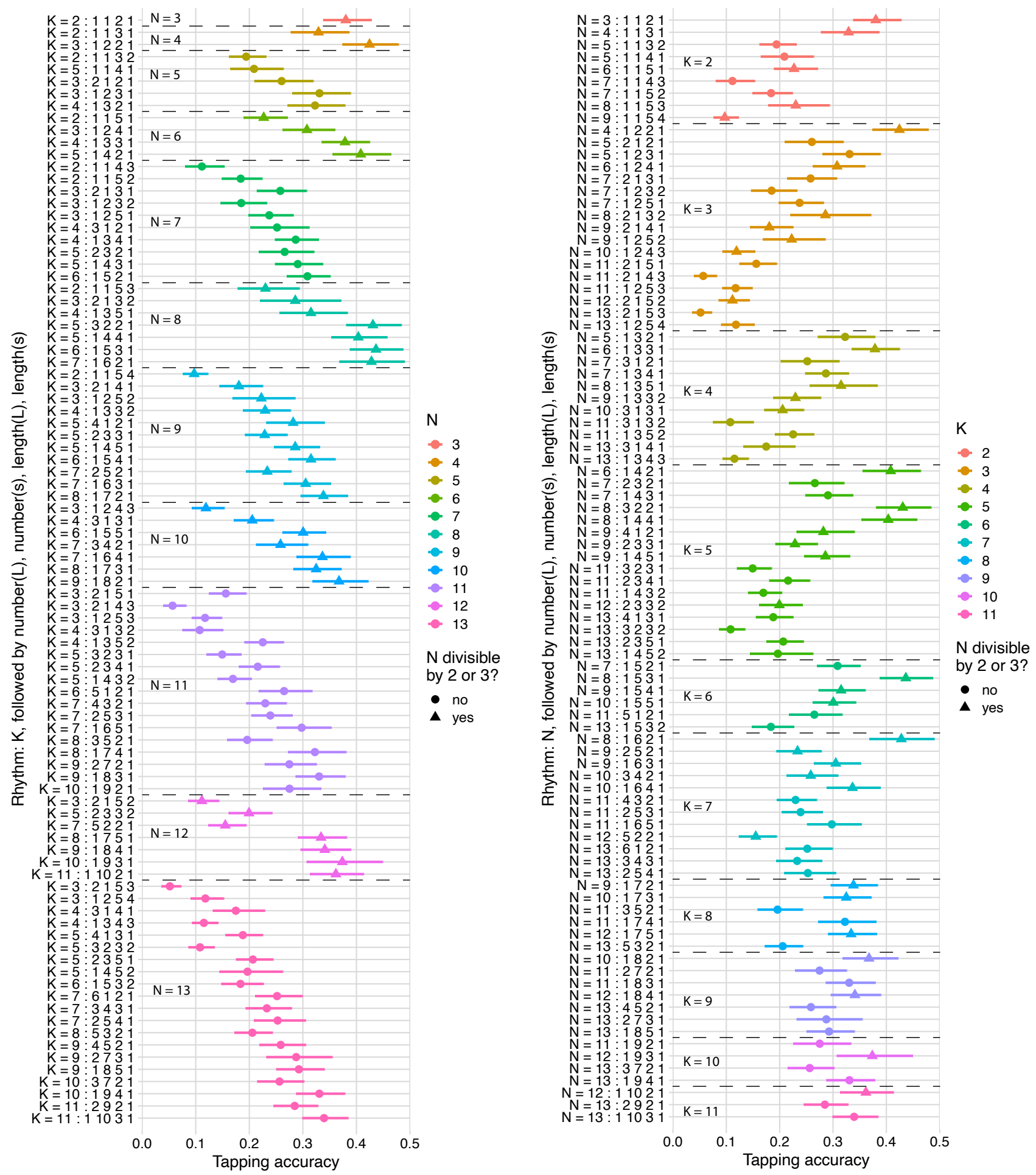

Figure 2. Tapping accuracy by rhythm (95\% credibility intervals): on the left, ordered by $N$; on the right, ordered by $K$. 



Figure 3. Velocity distributions smoothed across time (modulo the rhythmic period) of a subset of 11 of the 91 rhythms performed. Solid radii mark cued pulses; dashed radii mark uncued pulses. The data are normalized for each rhythm. 


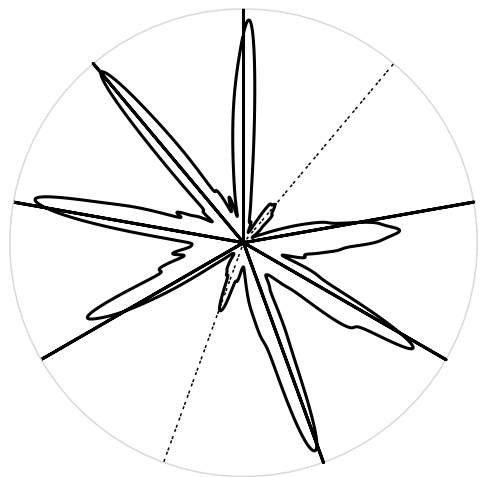

$K=7, N=9(2 L, 5 s)$

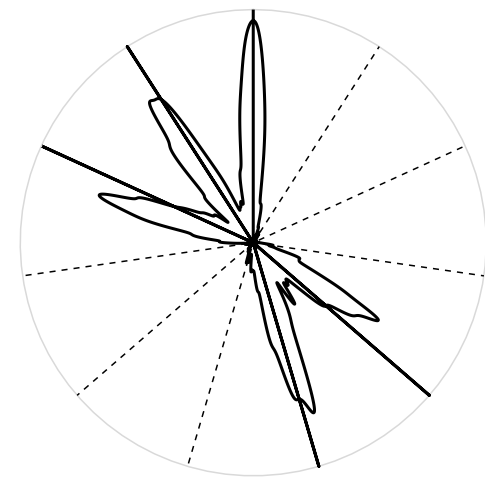

$K=5, N=11(2 L, 3 s)$

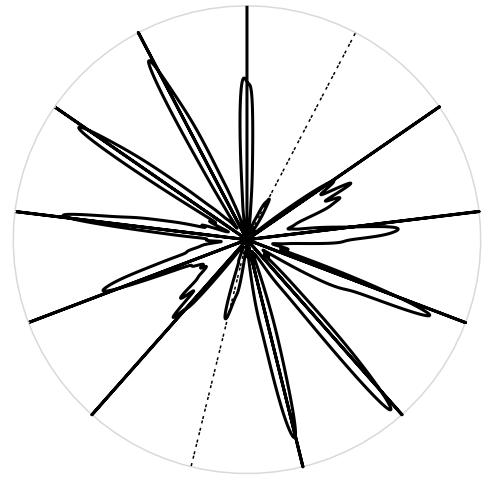

$K=11, N=13(2 L, 9 s)$

Figure 4. Velocity distributions smoothed across time (modulo the period) of three rhythms, all of which have two distinct clusters (groups) of cues. Solid radii mark cued pulses; dashed radii mark uncued pulses. The data are normalized for each rhythm.

\subsection{Performance-level tap accuracy}

\subsubsection{Results}

From the full set of performance-level and rhythm-level predictors detailed in Sections 2.2.1 and 2.2.2, the seven shown in Table 3 emerge after the variable selection process outlined in Section 2.3.2.

Table 3. Performance-level tap accuracy model. The effects are grouped by level (performance, rhythm). The "Hypothesis" column shows the hypothesis being tested, "Estimate" is the mean of the respective effect's posterior distribution, "l-90\% CI" and "u-90\% CI" show the effect's 90\% credibility interval (a 95\% CI is used for $R^{2}$ at the bottom), "Evid.Ratio" are the odds of the hypothesis being true, "Post.Prob" shows the probability the hypothesis is true (hence, Evid.Ratio = Post.Prob/(1-Post.Prob)). We qualify an evidence ratio $>10$ as "strong" evidence, an evidence ratio $>30$ as "very strong" evidence, and an evidence ratio $>100$ as "decisive". Full model summaries, including the group-level effects are provided in the Appendix. The two forms of Rsquared are calculated using the methods detailed in (Gelman et al., 2018); the cross-validated R-squared is calculated from the PSIS-LOO approximation of leave-one cross-validation (Vehtari et al., 2017).

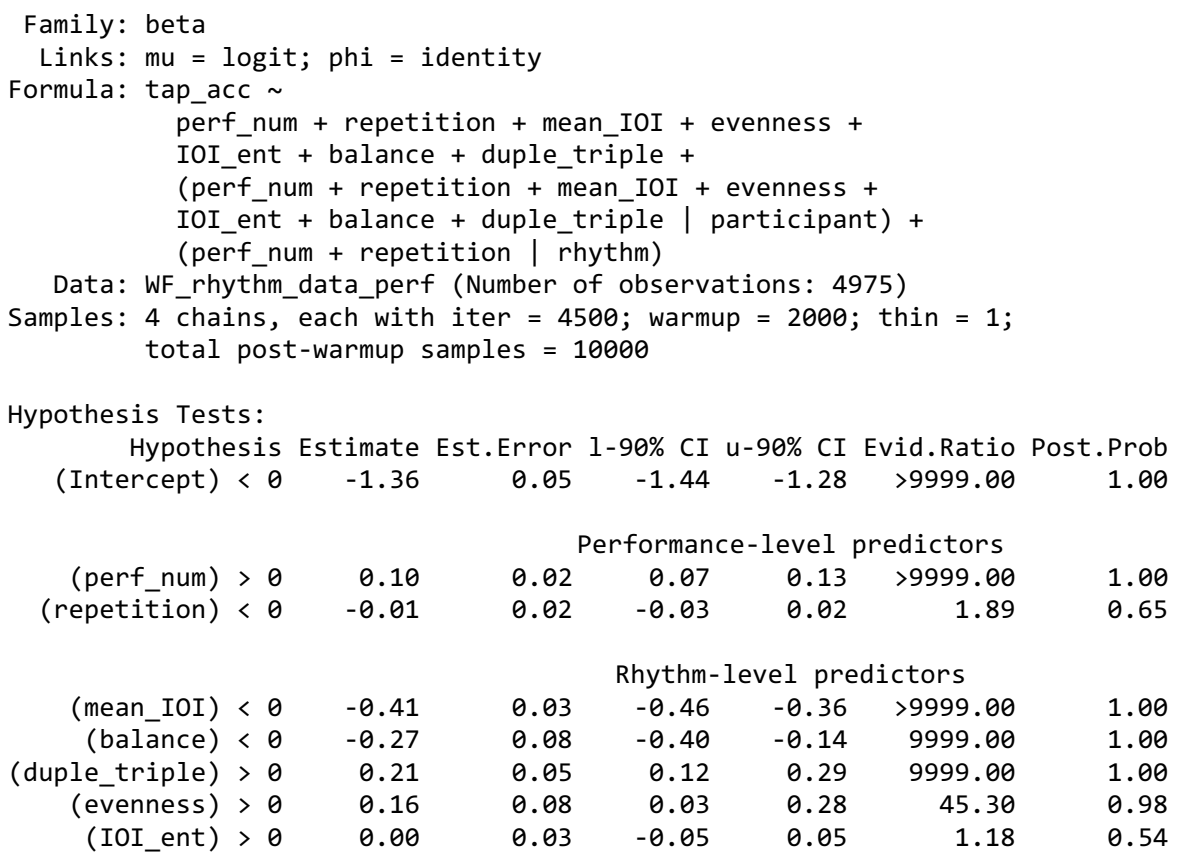




$\begin{array}{rrrrr}\text { R-squared Values: } & & & & \\ & \text { Estimate } & \text { Est.Error } & 1-95 \% \text { CI } & \text { u-95\% CI } \\ \text { Bayes R2 } & 0.67 & 0.01 & 0.66 & 0.68 \\ \text { Cross-validated R2 } & 0.63 & 0.01 & 0.61 & 0.65\end{array}$

\subsubsection{Discussion}

The intercept is -1.36 which means that, when all other predictors are at their mean, the predicted accuracy value is $\frac{\mathrm{e}^{-1.36}}{1+\mathrm{e}^{-1.36}}=0.20$. Accuracy - defined in Section $2.1 .1-$ is in the interval $[0,1]$, where 1 represents a perfect performance. To give an intuition, this roughly corresponds to tapping every cued pulse (and no uncued pulses) $20 \mathrm{~ms}$ early or late; alternatively, to about one quarter of taps being on-cue and three quarters off-cue (but, otherwise, perfectly timed). The group-level effects show that, after accounting for the fixed effects, there is moderate variation of accuracy (as represented by the intercept) between participants and rhythms - standard deviations of 0.38 and 0.28 , respectively. For example, a participant 1 standard deviation below the mean playing a rhythm 1 standard deviation below the mean would have an accuracy of $\frac{\mathrm{e}^{-(1.36+0.38+0.28)}}{1+\mathrm{e}^{-(1.36+0.38+0.28)}}=0.12$; a participant 1 standard deviation above the mean playing a rhythm 1 standard deviation above the mean would have an accuracy of $\frac{\mathrm{e}^{-1.36+0.38+0.28}}{1+\mathrm{e}^{-1.36+0.38+0.28}}=0.33$.

The effect size for mean_IOI is substantial. This confirms the previous observation that rhythms with more densely packed cues are tapped more accurately than rhythms with sparser cues.

As expected, balance has a negative impact - the more unbalanced a rhythm, the easier it is to tap. This fits the hypothesis that tapping around the rhythm's circular mean (a simple mechanism based on a lossy compression of the rhythm) is more likely to be successful than it is in a high-balance rhythm; furthermore, in unbalanced rhythms, not only is this circular mean more obvious, the preponderance of cues in one half of the period should generally facilitate position-finding within the rhythm.

Also as expected, duple_triple has a positive effect - rhythms that are periodic over a number of pulses that is divisible by two or by three are easier to tap. It suggests that being able to metricize rhythms into duple or triple time, provides some cognitive or motoric advantage; as discussed earlier, it may reduce working memory demands by allowing a complete rhythm to be chunked from familiar sub-rhythms which start on a regular metrical grid. This coefficient may also result from the greater prevalence of, hence participants' familiarity with, duple and triple meters in music (of course, the prevalence of such rhythms may arise because they can be more easily metricized).

evenness has a strongly evidenced weak effect; but in the opposite direction to that hypothesized (hypothesized, because uneven rhythms typically have one or more distinct clusters and gaps, and these should facilitate the identification of the grouping structure of the rhythm). This is likely because balance and evenness are so highly correlated (.94). The larger magnitude coefficient for balance, compared to evenness, shows that rhythms low in either balance or evenness are tapped more accurately; it is only when controlling for balance that there appears to be a small positive effect of evenness. But, given their high correlation, the precise values of these two coefficients will be variable across different data sets. Hence, the precise influence of balance relative to evenness and the effect of either conditioning on the other should be interpreted with some caution, and we can safely conclude only that rhythms with low balance or evenness are generally tapped more accurately.

Any effect of IOI_ent, which was anticipated to have a negative effect on tapping accuracy, is likely miniscule (its $95 \%$ credibility interval is -0.05 to 0.05 ). 
There is a small positive effect of perf_num; as participants progress through the experiment, they perform (slightly) better - a clear indication they develop general strategies to improve their performance. However, any effect of repetition (the same rhythm being performed for the second or third time) is almost certainly miniscule.

\subsection{Periodic pulse-level tap probability}

\subsubsection{Results}

As identified above, overall tapping accuracy is a function of tapping probability, velocity, and timing. In this subsection, we focus on the probability of tapping at each periodic pulse. After the variable selection process outlined in Section 2.3.2, we obtain the following set of predictors.

Table 4. Summary of the model of pulse-level tap probability. The three-line groupings, for every predictor, show Bayesian hypothesis tests for three effects of interest: in the first line, the predictor's effect on tapping bias (tapping incorrectly); in the second line, its effect on tapping discriminability; in the third line, its effect on tapping correctly (which is the sum of the previous two effects). The effects are grouped by level (performance, rhythm, pulse). The columns are the same as Table 3, as are the methods for calculating the R-squared values. Full model summaries, including the group-level effects are provided in the Appendix.

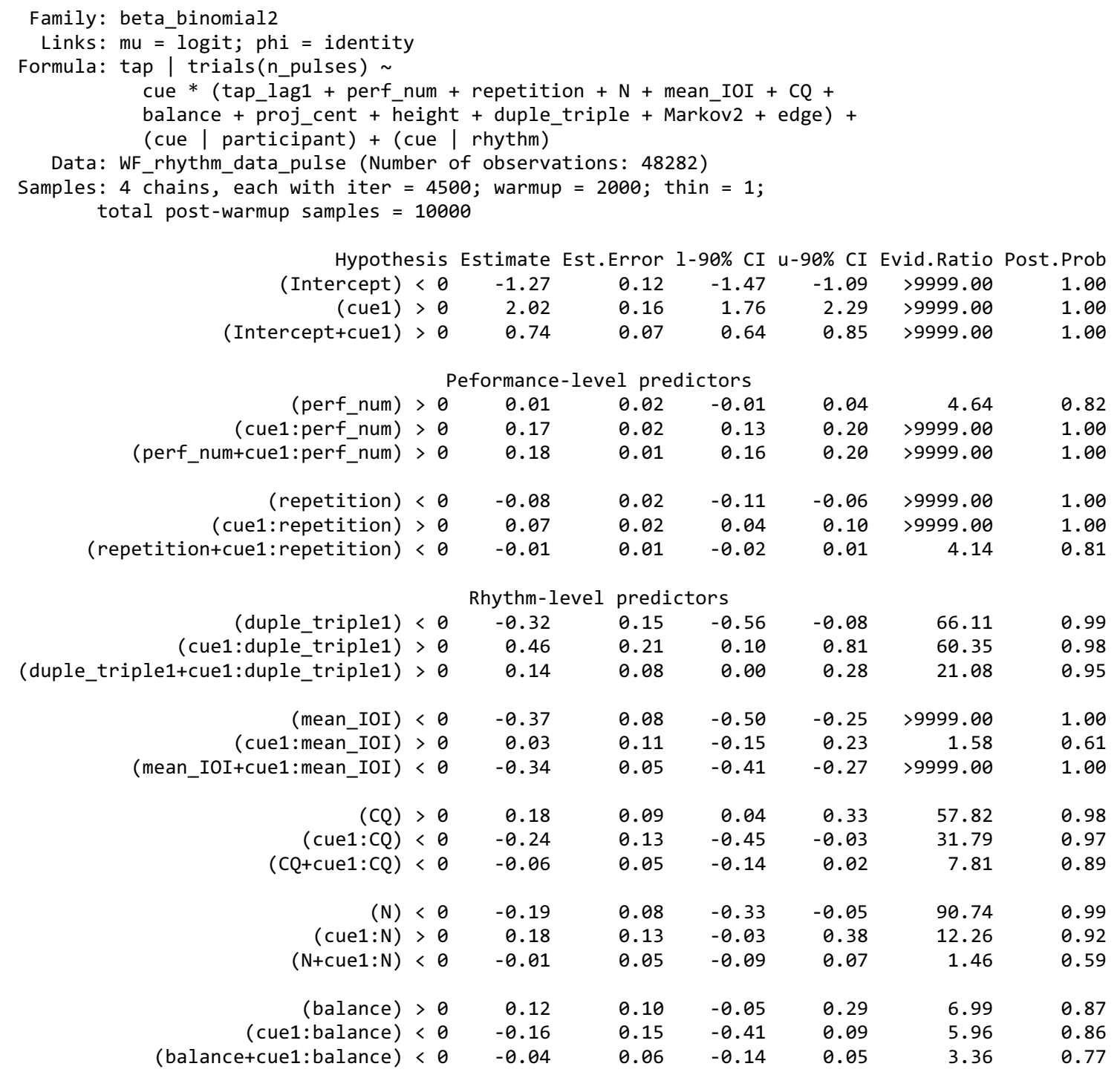




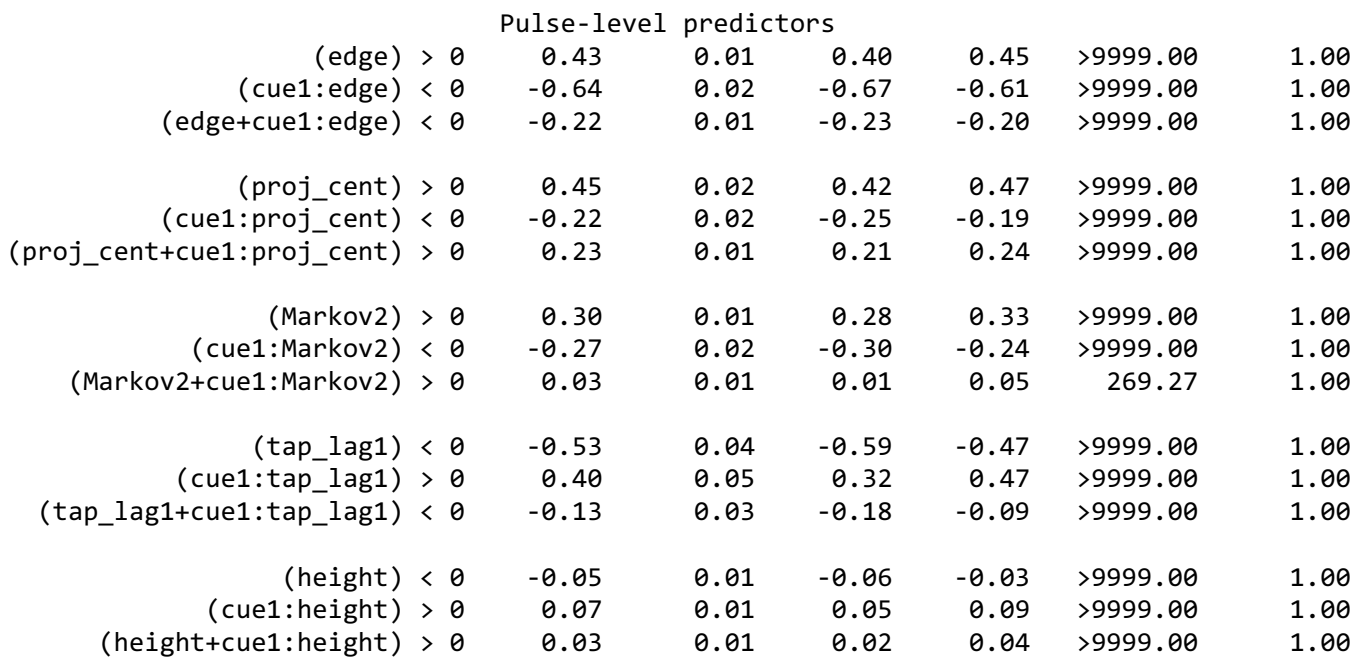

R-squared Values:

\begin{tabular}{|c|c|c|c|c|}
\hline & Estimate & Est.Error & $1-95 \%$ CI & u-95\% \\
\hline Bayes R2 & 0.85 & 0.00 & 0.84 & \\
\hline Cross-validated R2 & 0.85 & 0.00 & 0.85 & \\
\hline
\end{tabular}

The overall fit of the above "full" model - as assessed by the Bayesian $R^{2}$ values - is very good. It is interesting to compare its fit with those of the three reduced variants shown in Table 5: a model with only cue as a predictor (randomly varying by participant and rhythm); a full model but with all random effects removed; a model with only cue and no random effects. It is apparent that a model with only cue still has an acceptable fit (e.g., its crossvalidated $R^{2}$ is 0.75 ) which reflects its large effect size (2.02); adding random effects moderately improves the fit; adding all the predictors provides an additional small improvement in fit, which is highly significant.

Table 5. Measures of the fit of the full model reported in Table 4 compared with three reduced variants. The columns show two types of $R^{2}$ value (detailed in Table 3 ) and their cross-validated ELPD (expected log pointwise predictive density), which is an estimate of the model's ability to predict out-of-sample data. Higher ELPD values are better, and differences between ELPDs can be considered significant when they are at least twice their standard error.

\begin{tabular}{lrrrr}
\hline & Bayes R2 & CV R2 & $\Delta$ ELPD & SE \\
\hline Full model (with random effects) & 0.85 & 0.85 & 0.0 & 0.0 \\
Only cue (with random effects) & 0.83 & 0.83 & -2910.1 & 75.1 \\
Full model (no random effects) & 0.79 & 0.79 & -6979.9 & 118.3 \\
Only cue (no random effects) & 0.72 & 0.75 & -11014.8 & 136.4 \\
\hline
\end{tabular}

The conditional effects plots in Figure 5 show how each of the pulse-level predictors influences the probability of tapping incorrectly (when there is no cue) and correctly (when there is a cue) when all other predictors are at their mean. The more separated the two lines the greater participants' discrimination between cued and uncued pulses. For example, we can see that as the edge predictor increases (i.e., for cues at the edges of distinct clusters), discriminability drops markedly; slightly differently, as proj_cent increases (i.e., for cues close to an unbalanced rhythm's centroid), the overall bias for tapping on-cue and off-cue strongly increases, while discriminability reduces a smaller amount. 

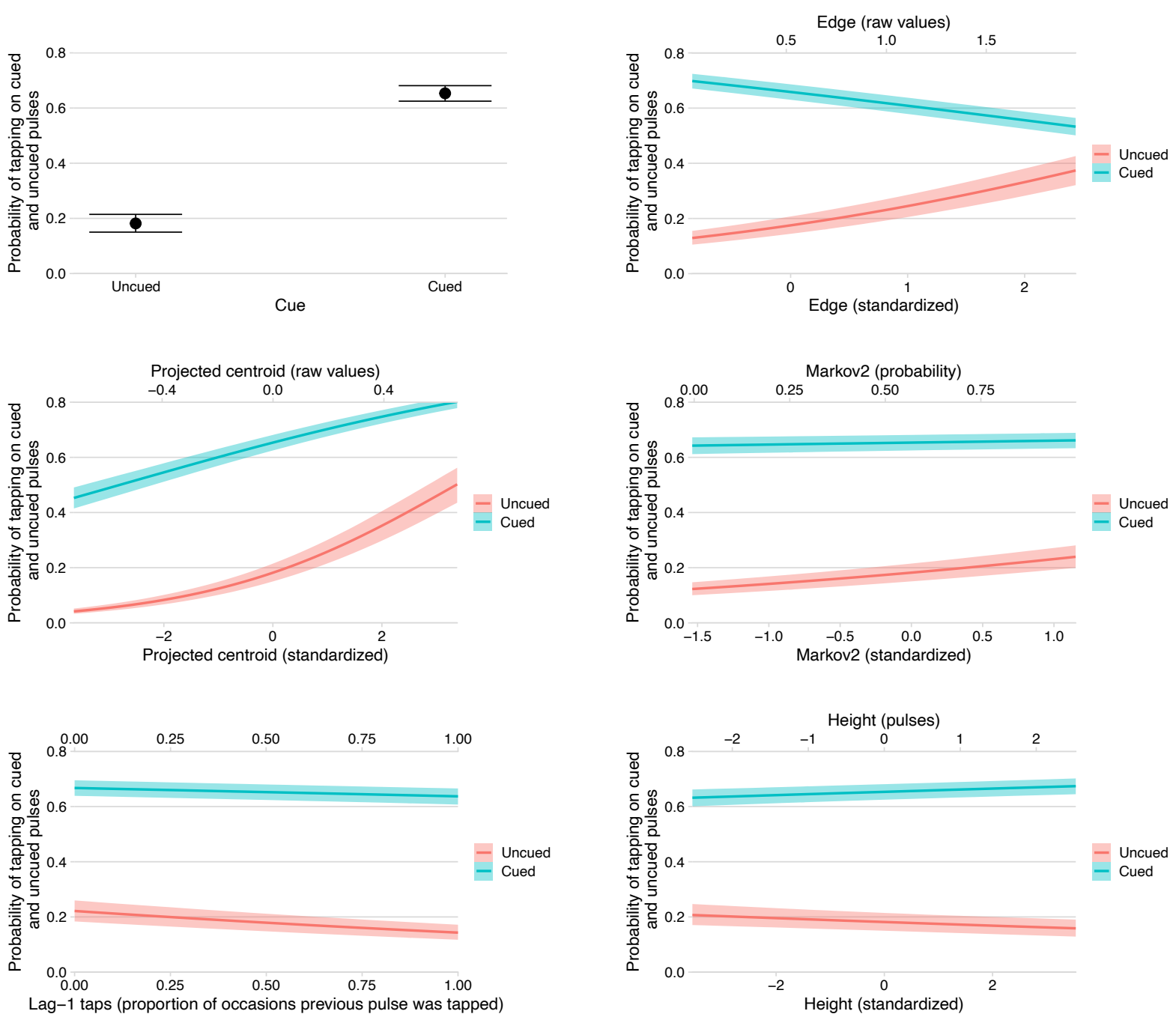

Figure 5. The effect of each pulse-level predictor on the probability of tapping on uncued (red) and cued (blue) pulses. Each effect is conditioned on all other predictors being at their mean values. The ribbons show $95 \%$ Bayesian credibility intervals.

\subsubsection{Discussion}

The meanings of the results for all the regression inputs are now verbally summarized and discussed, by level. Note that effects shown below are standardized (because all predictors were standardized prior to running the model); odds ratios (OR) are also included; unless specifically mentioned, all effects reported are "very strongly" evidenced in the model (evidence ratios $>30$ ).

Group-level effects. Tapping behaviour differs substantially between participants and rhythms. For example, discriminability has a standard deviation of 0.78 between participants (a 1 standard deviation difference, therefore, corresponds to an odds ratio of $\mathrm{e}^{0.78}=2.2$ ), and 0.96 between rhythms (a corresponding OR of 2.6).

Performance-level predictors. perf_num increases the discriminability of cues through increasing the number of cued taps, though the effect is relatively modest $(0.17, O R=1.18)$. The positive effect, on discriminability, of repetition is very small $(0.07, O R=1.08)$ and is achieved through reducing the number of uncued taps. In summary, participants slightly improve their general tapping strategies over the course of the experiment but show only marginal learning of individual rhythms between performances; although it is worth remembering that repeat performances of the rhythms were well separated because they occurred in different experimental blocks. 
Rhythm-level predictors. duple_triple has the expected large positive effect on discriminability $(0.46, O R=1.58)$ - cues are substantially better discriminated in rhythms that can be grouped into twos or threes without having to double the period of the rhythm (i.e., $N=3,4,6,8,9$, or 12 , rather than $N=5,7,11$, or 13 ). This may be due to such rhythms facilitating chunking (as detailed in Sec. 2.2) as well as their greater familiarity (such rhythmic periods being substantially more common in many musical traditions, including Western).

The effect of mean_IOI is interesting. As it decreases (i.e., cue density increases), the general probability of tapping (correctly or incorrectly) substantially increases (the effect on bias is $-0.37, O R=0.69$ ) but there is no evidence for an effect on discriminability.

$C Q$ - Carey's coherence quotient - seems to play a role here (it did not survive the variable selection process for the previous rhythm-level model). But its effect does not go in the direction that would be naturally hypothesized; higher CQ rhythms have lower discriminability $(-0.24, O R=0.78)$ because incorrect tapping increases $(0.18, O R=1.20)$, while there is only weak evidence of changes in tapping correctly.

Cues are more discriminable in longer (higher $N)$ rhythms $(0.18, O R=1.20)$; note that we are controlling for mean_IOI, so this is across different-length rhythms with similar cue density. This increase in discriminability results mostly from a lower probability of tapping incorrectly. It is not obvious what underlying mechanism would account for this effect of $N$.

The effect of balance is rather uncertain and there is no strong evidence of any specific directionality of the three effects of interest (note that all evidence ratios are below 10). This is probably because of the strong effect obtained from proj_cent, discussed below, which is weighted by balance.

Periodic pulse-level predictors. The effect of cue $(2.02, O R=7.52)$ is very strong. Participants are nearly 8 times more likely to tap correctly on-cue than to tap incorrectly offcue; this shows they were typically following the task we had set them and were learning the rhythms within each performance (remember the very small effect of repetition, which implies only a small amount of learning of specific rhythms occurred between performances). The strikingly high odds ratios may seem, at first sight, to contradict the relatively poor tapping accuracy values (tap_acc) predicted in the previous model and illustrated in Figure 2; but it is important to remember that the tap_acc dependent variable penalizes timing errors, while the tap_num dependent variable used in this model does not (so long as the tap falls into the $234 \mathrm{~ms}$ window centred on the pulse). So, although discrimination between cued and uncued taps is rather good, the timings of those taps is not. The standard deviations of the group-level (random) effect of cue by rhythm (0.96) and of cue by participant (0.78) show that the rhythms differed substantially in the discriminability of their cues, and participants differed substantially in their ability to discriminate cues.

edge represents a generalization of Povel's accentual weights. Povel's accents include pulses at the ends of groups of one or more cues, and at the starts of groups of three or more cues; edge also accents the starts and ends of groups of uncued pulses. The model shows that tap probabilities actually proceed in the opposite direction to the expected Povel accents: medial pulses (those not at the edges of clusters) are tapped more often, which confirms the observations made in Section 4.1. For cued pulses, the effect of being at the edge of a cluster - as quantified by $e d g e-$ decreases tapping $(-0.22, O R=0.81)$; for uncued pulses, the effect of being at the edge of a cluster of un-cued pulses - as quantified by edge - strongly increases tapping $(0.43, O R=1.53)$. This indicates that participants are aware of cueclusters but not sure when they will start: once they have heard the first cue in a cluster, they are confident the cluster has started and so they should now tap; given a long gap (a cluster of uncued pulses), they begin to anticipate the next cue-cluster is coming and so tap incorrectly before the cluster has started - the incorrectness of this tap perhaps then encouraging them to 
not tap in the next pulse hence missing a possible cue at the start of the cluster. In sum, participants are aware of the clusters but not sure exactly where they start or end.

If tap probabilities and tap accents are driven by the same mechanism, these results for edge run counter to conventional wisdom; for this reason, and despite the above plausible explanation, we felt it advisable to check whether these results may arise from how edge generalizes Povel's weights to uncued pulses. To do this, we tested two additional models of tapping probabilities; crucially, both were fitted only to cued pulses. One model contained the edge predictor, the other model replaced edge with three binary predictors indicating whether a cue was isolated (iso_accent), at the end of a group of two or more cues (end_accent), at the start of groups of three or more clusters (start_accent) - these being the precise criteria used by Povel. Both models are fully summarized in Appendices A. 9 and A. 10 but, in brief, both had an excellent fit to the data (cross-validated $R^{2}$ s of 0.80 ), and both confirmed, with very strong evidence, that cues at the edges of clusters were less likely to be tapped. The second new model showed that isolated cues were, as expected, tapped more. However, the extra level of detail provided by the three Povel indicators, compared to the single, hence more parsimonious, edge predictor, is questionable because crossvalidation shows the out-of-sample predictive performance of the two models cannot be reliably distinguished (although on balance of probabilities, the edge model is weakly favoured).

The effects for tap_lag 1 show that tapping on the previous pulse improves discriminability $(0.40, O R=1.49)$, principally by discouraging incorrect taps $(-0.53, O R=$ 0.59 ) on the current pulse (correct taps are discouraged by a somewhat smaller amount). (Note that tap_lag1 is not standardized like the other continuous variables; its standardized effects are given by multiplying its effects shown in Table 4 by the standard deviation of tap_lag 1 in the data, which is 0.348 .) The overall reduction in tap probability may be an artefact of the rhythms performed because the proportion of consecutive cues is 0.333 (and taps occur more commonly on cued pulses than on uncued pulses); it may also result from the short-term fatigue induced by making a tap, which discourages a subsequent tap (regardless of its correctness). The increase in discriminability may be due to a prior tap acting as a confirmation that the participant is at least attempting to perform the rhythm rather than "sitting it out".

proj_cent encourages participants to tap on cue $(0.23, O R=1.25)$ but encourages them even more to tap off-cue $(0.45, O R=1.56)$; hence reduces discriminability $(-0.22, O R=$ $0.80)$. It seems that although the information provided by the rhythm's centroid - tap close to strong rhythmic centroids; do not clap close to the opposite of strong rhythmic centroids - is very influential for behaviour, it is actually a rather poor tapping mechanism (for discriminability). As with $C Q$, this perhaps suggests that proj_cent may be a learned familiar and useful mechanism in more conventional rhythms - such as those with unsyncopated groups - but that it fails for the extended uneven rhythms used in this study.

Markov2 strongly encourages participants to tap off-cue $(0.30, O R=1.35)$ and so reduces discriminability $(-0.27, O R=0.76)$. This suggests that they were relying, in part, on a Markovian representation, or some similar lossy representation of the pattern in terms of how very short-term rhythmic figures tend to continue within that pattern. Interestingly, this effect seems to operate in one direction only: we did not find a tendency not to tap on cued pulses with low Markov2. A hybrid mechanism for remembering patterns that combines rote memory of a few memorable features with lossy mechanisms for the pattern's remaining features suggests a possible explanation for this asymmetric effect. It seems likely that a sound (i.e., a cue) that defies a sub-rhythmic regularity is more memorable than a "silence" that defies a sub-rhythmic regularity (just as onsets make a stronger impression than offsets). Using such a hybrid mechanism, a participant might - with relative ease - learn the 
surprising cues by rote, but perform more poorly at the surprising silences, misled by a lossy mechanism into tapping incorrectly.

The effect of height is very small - it marginally decreases off-cue taps $(-0.05, O R=$ $0.95)$ and marginally increases on-cue taps $(0.03, O R=1.03)$; hence marginally increases discriminability $(0.07, O R=1.07)$. Generally, for well-formed rhythms, pulses with high height are close to the ends of sequences of small IOIs; pulses with low height are close to the starts of sequences of small IOIs. This effect, therefore, suggests a predictive mechanism that pays particular attention to sequences of short-IOI cues - perhaps the number of cues in each cluster is learned and once the participant has recognized that that cluster has started, they can enact that knowledge. However, this effect is very small.

\subsection{Pulse-level tap velocity}

\subsubsection{Results}

The model for tap velocity was fitted only to pulse-level observations where a tap had occurred. For the tapped pulses' velocities, a Gaussian family model was used and values at the maximum possible MIDI velocity level (127) were treated as censored (i.e., they were, correctly, modelled as meaning $\geq 127$ ). Following the variable selection process (Section 2.3.2), the model summarised in Table 6 emerged.

Table 6. Summary of the model of pulse-level tap velocity. The two-line groupings, for every predictor except cue, show Bayesian hypothesis tests for two effects: in the first line, the predictor's effect on the velocity of incorrect taps; in the second line, its effect on the velocity of correct taps. The effects are grouped by level (performance, rhythm, pulse). The columns are the same as Table 3, as are the methods for calculating the Rsquared values. Full model summaries, including the group-level effects are provided in the Appendix.

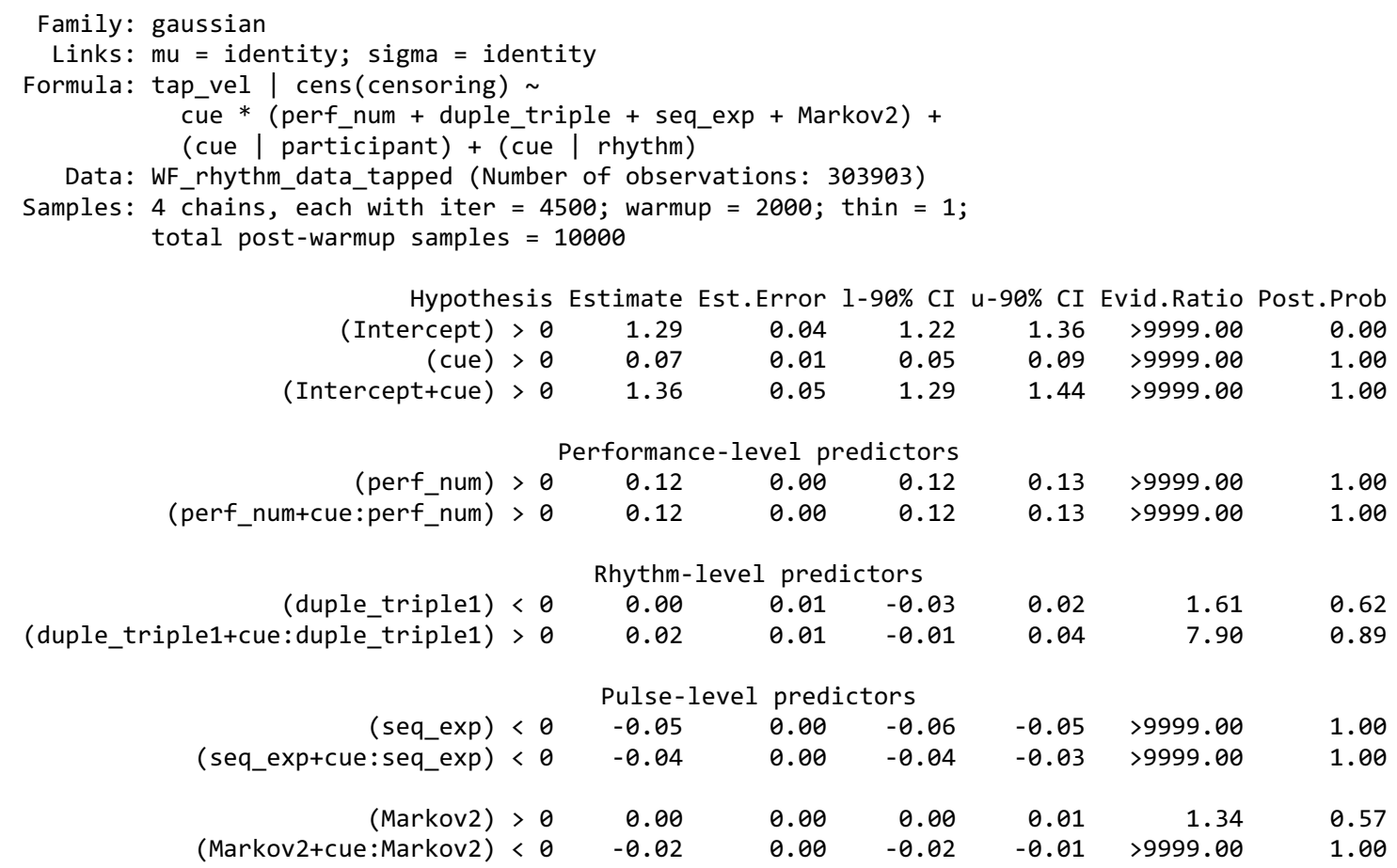

R-squared Values:

$\begin{array}{rrrrr} & \text { Estimate } & \text { Est. Error } & 1-95 \% \text { CI } & \text { u-95\% CI } \\ \text { Bayes R2 } & 0.62 & 0.00 & 0.62 & 0.62 \\ \text { Cross-validated R2 } & 0.58 & 0.00 & 0.58 & 0.58\end{array}$

\subsubsection{Discussion}

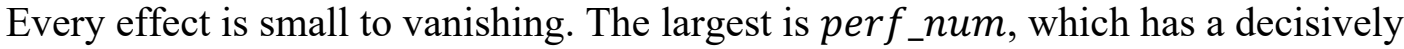
evidenced but small effect of 0.12 - with each successive performance, participants tend to 
tap slightly harder. The remaining predictors' effects - even cue - are vanishingly small. The model has impressive $R^{2}$ values but these are due mostly to the differences between participants as captured by the participant-level variation in the intercept, which has a standard deviation of 21.7 MIDI velocity units - some participants generally tap substantially harder than others. Variation between rhythms was trivial. (An otherwise equivalent model without group-level effects has Bayesian and cross-validated $R^{2}$ values of only 0.05 ).

It is interesting to note that edge does not make it through the variable selection process, so has weak predictive power for tap velocity. Hence, we have no evidence that the starts and ends of clusters are tapped harder than are medial cues. Once again, this does not support prior hypotheses in (Povel \& Essens, 1985) nor the tap velocities observed in Repp et al. (2005), where starts and ends of clusters were tapped harder than medial onsets. Perhaps Povel and Essen's accents are only manifested in simpler rhythms that have been fully learned or maybe, for our difficult rhythms, they relate more to perception than they do to tapping.

\subsection{Pulse-level tap timing error}

\subsubsection{Results}

For the signed tap-timing error, a truncated Gaussian family model was used; due to the assignment of each tap to its closest possible pulse and the deletion of any duplicate taps within a single pulse window, which is in the interval $[-117,117]$ milliseconds relative to each pulse, the data are truncated outside that interval and are modelled accordingly. Following the variable selection process (Sec. 2.3.2), the model summarised in Table 7 emerged.

Table 7. Summary of the model of pulse-level tap timing error. The two-line groupings, for every predictor except cue, show Bayesian hypothesis tests for two effects: in the first line, the predictor's effect on the timing of incorrect taps; in the second line, its effect on the timing of correct taps. The effects are grouped by level (performance, rhythm, pulse). The columns are the same as Table 3. Full model summaries, including the group-level effects are provided in the Appendix.

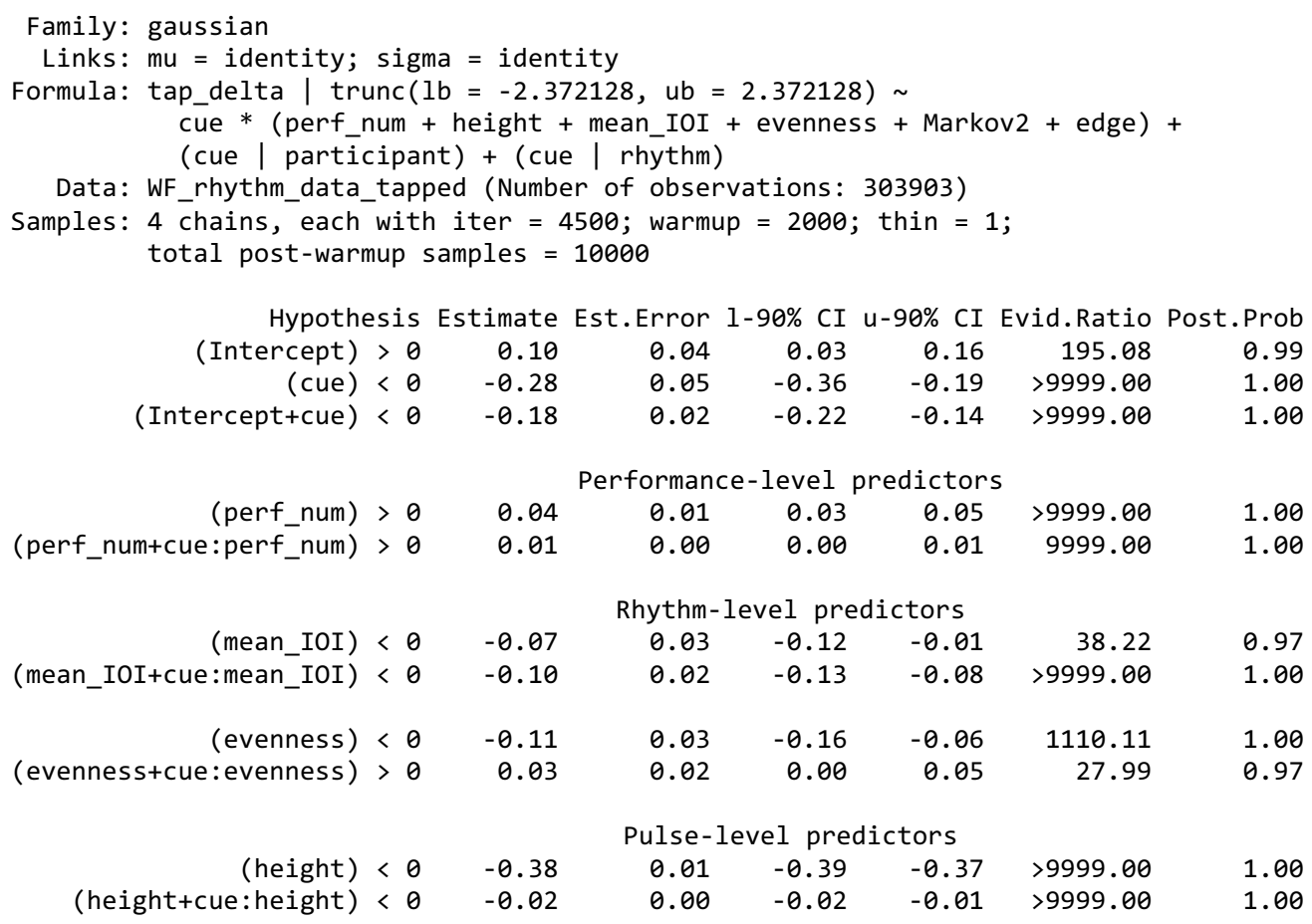




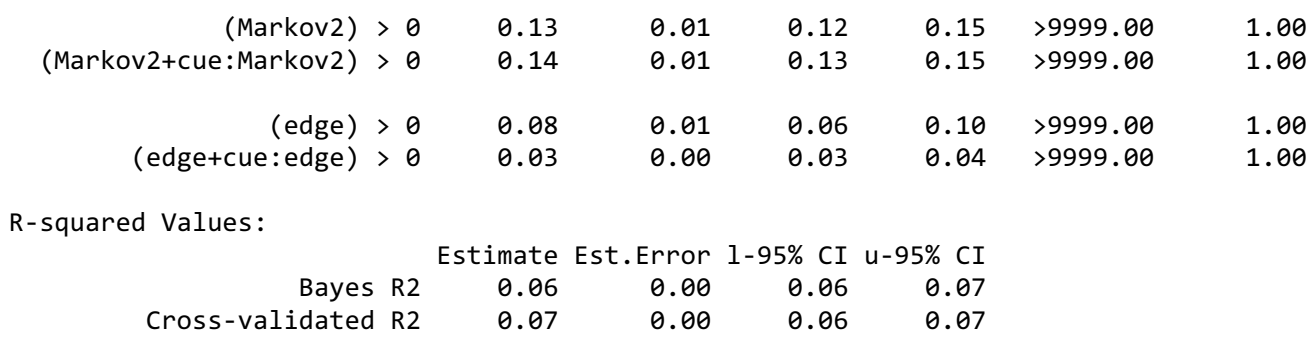

The model's overall fit to the data is poor, indicating that timing behaviour is not particularly influenced by between-participant or between-rhythm effects (the group-level standard deviations of the intercept and cue are, respectively, $9.4 \mathrm{~ms}$ and $14.3 \mathrm{~ms}$ by participant; $14.8 \mathrm{~ms}$ and $19.2 \mathrm{~ms}$ by rhythm). We do, however, obtain some predictors with non-trivial effects.

\subsubsection{Discussion}

Cued (correct) taps are earlier than uncued (incorrect taps). With all other predictors at their means or reference levels - a cued tap is predicted to occur about 9 milliseconds before the pulse; an uncued tap about 4.8 milliseconds after the pulse; a small but meaningful difference of 13.8 milliseconds. For simple isochronous rhythms, performers typically exhibit a negative mean asynchrony (for non-musicians, approximately $20-80 \mathrm{~ms}$ before the auditory cue (Aschersleben, 2002)), and this is indicative of the performers making anticipated rather than reactive taps (bearing in mind, of course, that a tap would have to be late by at least $150 \mathrm{~ms}$ to be reactive, (Repp, 2003)). Given that cued pulses are more likely to be anticipated than uncued (the tap probability model shows that cued pulses are tapped with substantially greater probability than uncued pulses), this suggests that earlier tap timings may be a useful proxy for the extent to which the tap is based on a strong prediction (regardless of whether that prediction is correct).

Uncued pulses with higher height are tapped moderately earlier (a unit increase in height has an effect of -0.38 , which corresponds to $-18.8 \mathrm{~ms}$ ). For well-formed patterns, uncued high height pulses come soon after a sequence of short IOIs; uncued low height pulses come shortly before a sequence of short IOIs (the length of the short-IOI sequence and the closer the pulse follows or precedes, the greater the magnitude of the height, although other factors also play a role). To the extent that tap timing is reflective of how strongly the participant has predicted that tap, these results suggest that participants more strongly anticipate a cue following a sequence of fast cues (i.e., a cue cluster). Typically, we would expect predicted taps to occur early - the negative mean asynchrony effect - but note that for incorrect taps (all else at the mean) the intercept predicts taps will be almost $5 \mathrm{~ms}$ late; the effect of height, which is substantial only for uncued taps, has the potential to turn this into an early tap for pulses where a tap is predicted, but predicted incorrectly. The tap probability model shows that these incorrect predictions of high height pulses are uncommon but, when they do occur, they occur early. The lack of an effect of height for cued pulses does not obviously replicate those of Repp et al. (2005) and Snyder et al. (2006) who found that, within groups, taps (only correct taps were assessed) occur progressively earlier.

Taps (correct and incorrect) are slightly later for pulses with greater Markov2 (an effect of 0.14 corresponds $6.7 \mathrm{~ms}$ ).

The effect of evenness on incorrect taps is small (an effect of -0.11 corresponds to $-5.5 \mathrm{~ms}$ ). All other effects are vanishingly small and so not discussed further.

\section{$5 \quad$ General discussion}

In order to perfectly synchronize to a rhythm, the performer must, somehow, store, retrieve, and process a lossless encoding of its cues' onset times. Clearly, the overall information 
content of any rhythm increases as its number of cues $(K)$ increases, and the rate at which that rhythm-level information is imparted is a function of the length of the rhythm $(N)$; the information content of any cue's position within a given rhythm is dependent on that rhythm's complexity (IOI_ent, int_ent), and the rate at which this information is imparted is given by the mean speed of the cues (mean_IOI). These, in combination with the coding mechanism used, will result in varying cognitive demands; for example, metricization should reduce demands on short-term memory, but only if the rhythm allows metricization over chunks comprising a sufficiently small amount of information (e.g., of no more than three pulses, duple_triple), and the cues are close enough in time to induce a strong beat (mean_IOI) (e.g., beats of around 500ms are easier to induce than those that are substantially longer or shorter).

Given that the distribution of possible rhythms heard by a person over their lifetime is non-uniform (i.e., it has lower than maximal entropy), there will be lossless codings that compress common rhythms at the expense of uncommon rhythms (it is a consequence of Shannon's source coding theorem that a lossless coding that reduces the size common items must increase the size of rare items). Any such lossless coding that can be performed by the brain may, therefore, reduce the subsequent cognitive demand of common rhythms whilst increasing the demand of unusual rhythms.

For rhythms that are too complex to be losslessly compressed sufficiently to avoid overwhelming cognitive resources, or rhythms whose rarity means they are not helped by established lossless encodings, we may find lossy encodings playing a role. Such encodings do not perfectly reproduce the rhythm - indeed, they may be crude and simplistic - but they can aim to increase the probability of correctly discriminating between cued and uncued pulses. For example, simple statistical summaries such as the circular mean and variance of the rhythm can allow for a rough guess of when a cue is more likely to occur (proj_cent) (and, clearly, this mechanism would work better the more unbalanced the rhythm, balance). An assessment of cue density (mean_IOI) may modify the criterion at which a tap is made if cues are densely packed, the criterion for tapping reduces. Similarly, approximate knowledge of the positions of groups and gaps (clusters of cued and uncued pulses) can facilitate discrimination ( $\mathrm{edge}$, height) - even if a performer is not sure exactly when each cue occurs, if a group is heard to have started, the next pulse is probably a good time to tap (this mechanism is more effective in more uneven rhythms because these have more obvious groups, evenness). Also, knowledge of a rhythm's distribution of interonset intervals (IOI_ent, int_ent) means that guessing the most common interval is more likely to be correct than a completely random guess (and, clearly, this mechanism works better the lower the IOI or interval entropy of the rhythm). Related to this, remembering small common patterns within each rhythm - for example, $n$-grams of consecutive pulses (Markov2,3,4,...) - may guide tapping decisions; even if imperfectly. Another possible form of lossy encoding may be achieved through inferring approximate positions of cues from the rhythm's generic structure; for instance, in the "pentatonic" rhythm (1 $\left.\begin{array}{lllllllllll}0 & 1 & 0 & 1 & 0 & 0 & 1 & 0 & 1 & 0 & 0\end{array}\right)$, the temporal interval between two consecutive cues is always smaller than the interval between two cues with a single intervening cue (as quantified by $C Q$ ); in other rhythms, these straightforward relationships do not hold. A final lossy encoding that may play a role is beat-induced tapping ( $A P M$ and seq_exp); if the cues are arranged in a pattern such that many of them are a subset of, or subsequence of, an isochronous beat, it makes sense to tap along with that isochronous beat, because it is easy to tap an isochronous beat and you will be correct much of the time.

Amongst these possible mechanisms, we see a strong effect of duple_triple on discriminability which may result from two different - but probably causally intertwined mechanisms: the reduction in cognitive load achieved by metricizing the rhythm into chunks with two or three pulses; and the greater familiarity of such rhythms. With regard to the 
latter, our performers are culturally familiar with duple and triple time (particularly the former) and will likely have their expectations "thrown" by periods that last one pulse more or one pulse less than commonly heard. The absence of any notable effect of $A P M$ (which did not make it through the variable selection process in any of the models) or of seq_exp suggests that our participants did not make direct use of metricization; they did not prefer tapping, or tap harder, at cues occurring in time with isochronous beats that might have been plausibly induced by the rhythmic cues. This suggests that the mechanism encoded by duple_triple is, for these rhythms and performers, mostly related to familiarity of the rhythms' periods $(N)$.

As shown in the first model, the effect of mean_IOI on rhythm-level accuracy is striking; however, the tap probability model shows that this predictor does not have a meaningful effect on cue discriminability. The accuracy effect arises, instead, from increased tap-timing errors when the mean interonset interval is larger (the correlation between pulse-level absolute timing error and mean_IOI is 0.19 ). The tap probability model shows that densely packed cues strongly encourage tapping regardless of their correctness. This seems to be an example of a crude behavioural mechanism: "this rhythm is dense so I am better off tapping now, unless I am very sure I shouldn't be tapping now" and, of course, the converse: "this rhythm is sparse so I am better off not tapping now, unless I am very sure I should be tapping now."

Another example of a simplistic mechanism guiding tapping behaviour is shown by the remarkably strong effect of the edge predictor on discriminability: pulses close to the starts and ends of long groups and gaps are typically tapped with considerably less discrimination than are pulses away from these edges (this is clear in the descriptive plots, Figures 3 and 4). It seems that our performers are aware there are groups of cues but unsure of their precise start time or their length; so they frequently tap, incorrectly, before the group has started or wait for confirmation that the group has started and then start tapping with gusto (but starting later than they should have) until they suspect the group is about to end or, indeed, hear that it has ended (hence they tap one more time than cued). Hence discriminability around the boundaries of groups is strongly reduced. This is a crude predictive mechanism, but one that works well for pulses in the centres of groups, which are often larger in number than the poorly discriminated pulses at the edges of groups; so, this is an overall reasonable approach for the participants to take. We also find no effect of edge on tap velocity. Together, these findings seem to contradict Povel's model for metrical accenting being stronger at the starts and ends of groups (Povel \& Essens, 1985) and the results of Repp et al. (2005), but they are unsurprising if the groups' start times and lengths are remembered only to a low order of approximation.

Two other grouping related features seem to play a role: unbalanced rhythms are tapped moderately more accurately and with better discrimination; and pulses close to the circular mean are tapped substantially more often. The former result parallels findings that unbalanced rhythms are more commonly classed as having been heard before (regardless of whether or not they have) and are generally preferred (Milne \& Herff, 2020). The results here suggest that participants are able to approximate the rhythm's circular mean and use that knowledge to guide their tapping: "the mean is about here so tap about now!" (We do not intend these quotation marks to imply this is a conscious strategy taken by the participant; it may be a manifestation of a latent cognitive bias towards tapping in this higher cue-density region of the rhythm, perhaps tapping multiple times in this region.) In terms of discriminability, however, this is not a wholly successful approach: close to the mean, incorrect taps increase more than correct taps (conversely, of course, tapping has more discrimination distant from the mean). The fact that imbalance has a moderately positive effect perhaps shows that, despite this intemperate tapping behaviour, a rhythm with two 
clearly defined "halves" (one "full", one "empty") may facilitate position-finding (knowing where one is) within the rhythm.

A surprising finding is that the coherence quotient $(C Q)$ has a negative effect on discriminability, mostly though increasing incorrect taps. We would expect it to be easier to locate cues in rhythms with high CQ; this is because - for high-CQ rhythms - counting the distance between cues give constraints on relative IOI sizes. Perhaps, for our 91 rhythms, the coherence quotient is strongly correlated with some unidentified feature that strongly affects discrimination, but it is not clear what that would be.

Although IOI entropy does not obviously influence tapping, it seems that small distinctive features in the rhythms - common trigrams of pulses, cued or uncued (Markov2) - are remembered and guide tapping decisions; even though this often results in uncued pulses being incorrectly tapped.

Cultural exposure to rhythms influences rhythmic tapping behaviour and which rhythms can be tapped accurately (Cameron et al., 2015; Jacoby \& McDermott, 2017; Polak et al., 2018). Given the music-cultural homogeneity of our participants, we cannot determine whether the mechanisms we identify above are moderated by cultural exposure. Most of our rhythms would have been unfamiliar to our participants - particularly those where $N$ is factorized by a prime higher than 3 - and it is plausible that a person familiar with such $N$ (e.g., a Balkan familiar with aksak) may have performed them more accurately than most of our participants. Furthermore, all but six of our participants had less than five years of regular musical practice. It is likely that trained musicians - particularly those with experience of unusual rhythms - would approach the task differently; for example, explicitly counting out the rhythms or making use of lossless encodings that do not so strongly penalize these complex rhythms. Having said that, a personal observation from two of this paper's authors, both of whom have experience playing complex rhythms, is that these rhythms are hard to replicate purely from hearing them, but they become substantially easier to perform if notation is provided.

We reiterate that these production-task findings can only be indicative of underlying cognitive mechanisms and of the perception of rhythms. In sum, the results indicate that, for these mostly difficult and unusual rhythms, participants are disrupted by unfamiliar period lengths (as quantified by duple_triple) and, to a large extent, make use of crude representations of the rhythm: notably, its density (as quantified by mean_IOI), its circular mean and variance (as quantified by balance and proj_cent), the approximate positions of groups of cues (as quantified by edge), and low-order Markov approximations (as quantified by Markov2). Furthermore, these cognitive mechanisms are often counterproductive for discriminating between cued and uncued pulses, which was the task participants were asked to undertake. It also seems that these are quite different to mechanisms - such as metricization and emphasizing group boundaries - thought to guide tapping behaviours in learned and familiar rhythms; this is further underlined by the negative mean asynchrony of taps being much smaller than is typical for simple rhythms.

These findings increase our understanding of the types of lossy encodings used to internally represent unfamiliar and complex periodic temporal patterns. From a musical perspective, they are particularly relevant to understanding how listeners perceive the complex (and possibly unfamiliar) rhythms found in many non-Western musical traditions and within more experimental Western genres.

\section{Funding}

Dr Andrew Milne is the recipient of an Australian Research Council Discovery Early Career Researcher Award (project number DE170100353) funded by the Australian Government. 


\section{CRediT authorship contribution statement}

Andrew J. Milne: Conceptualization (equal), Methodology (lead), Software (lead), Validation, Formal analysis, Investigation, Data Curation, Writing - original draft, Writing review \& editing (lead), Visualization. Roger T. Dean: Conceptualization (equal), Methodology (supporting), Writing - review \& editing (supporting). David Bulger: Methodology (supporting), Software (supporting), Writing - review \& editing (supporting).

\section{Declaration of competing interest}

The authors declare no competing interests.

\section{References}

Amiot, E. (2009). Discrete Fourier transform and Bach's good temperament. Music Theory Online, 15(2).

Aschersleben, G. (2002). Temporal control of movements in sensorimotor synchronization. Brain and Cognition, 48(1), 66-79.

Attneave, F. (1955). Symmetry, information, and memory for patterns. The American Journal of Psychology, 68(2), 209-222.

Balzano, G. J. (1982). The pitch set as a level of description for studying musical perception. In M. Clynes (Ed.), Music, Mind, and Brain: The Neuropsychology of Music (pp. 321351): Plenum Press.

Barr, D. J., Levy, R., Scheepers, C., \& Tilyc, H. J. (2013). Random effects structure for confirmatory hypothesis testing: Keep it maximal. Journal of Memory and Language, 68(3), 255-278.

Bolton, T. L. (1894). Rhythm. American Journal of Psychology, 6(2), 145-238.

Browne, R. (1981). Tonal Implications of the diatonic set. In Theory Only: Journal of the Michigan Music Theory Society, 5, 3-21.

Bürkner, P.-C. (2017). brms: An R package for Bayesian multilevel models using Stan. Journal of Statistical Software, 80(1), 1-28.

Bürkner, P.-C. (2018). Advanced Bayesian multilevel modeling with the R package brms. The R Journal, 10(1), 395-411.

Cameron, D. J., Bentley, J., \& Grahn, J. A. (2015). Cross-cultural influences on rhythm processing: reproduction, discrimination, and beat tapping. Frontiers in Psychology, 6(366).

Carey, N. (2002). On coherence and sameness, and the evaluation of scale candidacy claims. Journal of Music Theory, 46(1/2), 1-56.

Carey, N. (2007). Coherence and sameness in well-formed and pairwise well-formed scales. Journal of Mathematics and Music, 1(2), 79-98.

Carey, N., \& Clampitt, D. (1989). Aspects of well-formed scales. Music Theory Spectrum, 11(2), 187-206.

Carpenter, B., Gelman, A., Hoffman, M. D., Lee, D., Goodrich, B., Betancourt, M., . . . Riddell, A. (2017). Stan: A probabilistic programming language. Journal of Statistical Software, 76(1), 10.18637/jss.v18076.i18601.

Clayton, M. (2020). Theory and practice of long-form non-isochronous meters: The case of the North Indian rūpak tāl. Music Theory Online, 26(1).

Collier, G. L., \& Wright, C. E. (1995). Temporal rescaling of simple and complex ratios in rhythmic tapping. Journal of Experimental Psychology: Human Perception and Performance, 21(3), 602-627.

Cycling '74. (2021). Max [Computer software]. Cycling '74.

http://cycling74.com/products/max/ 
Dean, R. T., Bulger, D., \& Milne, A. J. (in press). On the roles of complexity and symmetry in cued tapping of well-formed complex rhythms: Descriptive statistics and time series analysis. Music Perception: An Interdisciplinary Journal, 39.

Eck, D. (2006). Identifying metrical and temporal structure with an autocorrelation phase matrix. Music Perception, 24(2), 167-176.

Essens, P. J., \& Povel, D.-J. (1985). Metrical and nonmetrical representations of temporal patterns. Perception \& Psychophysics, 37(1), 1-7.

Fracile, N. (2003). The aksak rhythm, a distinctive feature of the Balkan folklore. Studia Musicologica Academiae Scientiarum Hungaricae, 44(1), 191-204.

Fraisse, P. (1946). Contribution à l'étude du rythme en tant que forme temporelle. Journal de psychologie normale et pathologique, 39, 283-304.

Fraisse, P. (1956). Les Structures Rhythmiques. Publications Universitaires.

Friberg, A., \& Sundberg, J. (1995). Time discrimination in a monotonic, isochronous sequence. The Journal of the Acoustical Society of America, 98(5), 2524-2531.

Fripp, R. (1973). Larks' Tongues In Aspic, Part Two. [Recorded by King Crimson]. On Larks' Tongues In Aspic [LP].

Frith, F. (1974). Ruins. [Recorded by Henry Cow]. On Unrest [LP].

Garner, W. R. (1974). The Processing of Information and Structure. Lawrence Erlbaum Associates.

Gelman, A., Goodrich, B., Gabry, J., \& Vehtari, A. (2018). R-squared for Bayesian regression models. The American Statistician, 73(3), 307-309.

Goldberg, D. (2015). Timing variations in two Balkan percussion performances. Empirical Musicology Review, 4(10), 305-328.

Hammill, P. (1976). Meurglys III, The Songwriter's Guild. [Recorded by Van der Graaf Generator]. On World Record [LP].

Harry, B., \& Keller, P. E. (2019). Tutorial and simulations with ADAM: An adaptation and anticipation model of sensorimotor synchronization. Biological Cybernetics, 113, 397421.

Hearne, L. M. (2020). The Cognition of Harmonic Tonality in Microtonal Scales.

Hesselink, N. D. (2013). Radiohead's "Pyramid Song": Ambiguity, rhythm, and participation. Music Theory Online, 19(1).

Jacoby, N., \& McDermott, J. H. (2017). Integer ratio priors on musical rhythm revealed cross-culturally by iterated reproduction. Current Biology, 27(3), 359-370.

Jones, M. R., \& Pfordresher, P. Q. (1997). Tracking musical patterns using joint accent structure. Canadian Journal of Experimental Psychology, 51(4), 271-290.

Krause, V., Pollok, B., \& Schnitzler, A. (2010). Perception in action: The impact of sensory information on sensorimotor synchronization in musicians and non-musicians. Acta Psychologica, 1333(1), 28-37.

Krumhansl, C. L. (1983). Perceptual structures for tonal music. Music Perception, 1(1), 2862.

Makowski, D., Ben-Shachar, M. S., Chen, S. H. A., \& Lüdecke, D. (2019). Indices of effect existence and significance in the Bayesian framework. Frontiers in Psychology, 10, 2767.

Mardia, K. V. (1972). Statistics of Directional Data. Academic Press.

McLaughlin, J. (1971). The Dance of Maya. [Recorded by Mahavishnu Orchestra]. On The Inner Mounting Flame [LP].

Milne, A. J. (2018). Linking sonic aesthetics with mathematical theories. In A. McLean \& R. T. Dean (Eds.), The Oxford Handbook of Algorithmic Music (pp. 155-180). Oxford University Press.

Milne, A. J., Bulger, D., \& Herff, S. A. (2017). Exploring the space of perfectly balanced rhythms and scales. Journal of Mathematics and Music, 11(2-3), 101-133. 
Milne, A. J., Bulger, D., Herff, S. A., \& Sethares, W. A. (2015). Perfect balance: A novel principle for the construction of musical scales and meters. Mathematics and Computation in Music, London.

Milne, A. J., \& Dean, R. T. (2016). Computational creation and morphing of multilevel rhythms by control of evenness. Computer Music Journal, 40(1), 35-53.

Milne, A. J., \& Herff, S. A. (2020). The perceptual relevance of balance, evenness, and entropy in musical rhythms. Cognition, 203, 104233.

Modartt. (2021). Pianoteq 5 [Computer software]. Modartt. https://www.modartt.com

Moelants, D. (2006). Perception and performance of aksak metres. Musicae Scientiae, 10(2), $147-172$.

Newen, A. (2017). What are cognitive processes? An example-based approach. Synthese, 194(11), 4251-4268.

Ollen, J. E. (2006). A criterion-related validity test of selected indicators of musical sophistication using expert ratings.

Osborn, B. (2017). Everything in Its Right Place: Analyzing Radiohead. Oxford University Press.

Parncutt, R. (1994). A perceptual model of pulse salience and metrical accent in musical rhythms. Music Perception, 11(4), 409-464.

Pearce, M. (2018). Statistical learning and probabilistic prediction in music cognition: Mechanisms of stylistic enculturation. Annals of the New York Academy of Sciences, $1423,378-395$.

Pelofi, C., \& Farbood, M. M. (2021). Asymmetry in scales enhances learning of new musical structures. Proceedings of the National Academy of Sciences, 118(31).

Piironen, J., Paasiniemi, M., \& Vehtari, A. (2020). Projective inference in high-dimensional problems: Prediction and feature selection. Electron. J. Statist., 14(1), 2155-2197.

Piironen, J., \& Vehtari, A. (2017). Comparison of Bayesian predictive methods for model selection. Statistics and Computing, 27(3), 711-735.

Polak, R. (2010). Rhythmic feel as meter: Non-isochronous beat subdivision in jembe music from Mali. Music Theory Online, 16(4).

Polak, R., Jacoby, N., Fischinger, T., Goldberg, D., Holzapfel, A., \& London, J. (2018). Rhythmic prototypes across cultures: A comparative study of tapping synchronization. Music Perception: An Interdisciplinary Journal, 36(1), 1-23.

Povel, D.-J. (1981). Internal representation of simple temporal patterns. Journal of Experimental Psychology: Human Perception and Performance, 7(1), 3-18.

Povel, D.-J., \& Essens, P. (1985). Perception of temporal patterns. Music Perception: An Interdisciplinary Journal, 2(4), 411-440.

Povel, D.-J., \& Okkerman, H. (1981). Accents in equitone sequences. Perception and Psychophysics, 30(6), 565-572.

Pressing, J. (1983). Cognitive isomorphisms between pitch and rhythm in world musics: West Africa, the Balkans and Western tonality. Studies in Music, 17, 38-61.

Repp, B. H. (2003). Rate limits in sensorimotor synchronization with auditory and visual sequences: The synchronization threshold and the benefits and costs of interval subdivision. Journal of Motor Behavior, 35(4), 355-370.

Repp, B. H., London, J., \& Keller, P. E. (2005). Production and synchronization of uneven rhythms at fast tempi. Music Perception: An Interdisciplinary Journal, 23(1), 61-78.

Repp, B. H., London, J., \& Keller, P. E. (2011). Perception-production relationships and phase correction in synchronization with two-interval rhythms. Psychological Research, 75, 227-242.

Repp, B. H., London, J., \& Keller, P. E. (2012). Distortions in reproduction of two-interval rhythms: When the "attractor ratio" is not exactly 1:2. Music Perception, 30(2), 205-223. 
Repp, B. H., London, J., \& Keller, P. E. (2013). Systematic distortions in musicians' reproduction of cyclic three-interval rhythms. Music Perception: An Interdisciplinary Journal, 30(3), 291-305.

Repp, B. H., \& Su, Y.-H. (2013). Sensorimotor synchronization: A review of recent research (2006-2012). Psychonomic Bulletin and Review, 20, 403-452.

Snyder, J. S., Hannon, E. E., Large, E. W., \& Christiansen, M. H. (2006). Synchronization and continuation tapping to complex meters. Music Perception: An Interdisciplinary Journal, 24(2), 135-146.

Toussaint, G. T. (2002). A mathematical analysis of African, Brazilian, and Cuban clave rhythms. Proceedings of BRIDGES: Mathematical Connections in Art, Music, and Science,

Toussaint, G. T. (2003). Classification and phylogenetic analysis of African ternary rhythm timelines. In ISAMA-BRIDGES Conference Proceedings (pp. 25-36).

Vehtari, A., Gelman, A., \& Gabry, J. (2017). Practical Bayesian model evaluation using leave-one-out cross-validation and WAIC. Statistics and Computing, 27(5), 1413-1432.

Yorke, T., Greenwood, C., O'Brien, E., Greenwood, J., \& Selway, P. (2001). Pyramid Song. [Recorded by Radiohead]. On Amnesiac [LP]. 


\section{A. 1 Performance-level tap accuracy method and calculation}

We measured our performers' tap times and velocities, and the stimulus times. For each 30second performance, it is useful to find a way to summarize these data to produce a single number that serves as an effective estimate of the tapping accuracy of each 30 -second performance. For our purposes, a measure of tapping accuracy ideally fulfils the following criteria:

- Penalizes non-performance. For example, if a performer is silent for the first half of the performance, but then taps perfectly for the second half, it seems reasonable that their tapping accuracy should be half that of a performer who taps perfectly throughout the entire stimulus.

- Monotonically and symmetrically penalizes timing errors. The greater the difference between the tap time and the stimulus time, the lower the accuracy. For example, if a performer taps perfectly except for one cue where they tap $50 \mathrm{~ms}$ early this should result in the same accuracy as if they tap $50 \mathrm{~ms}$ late; furthermore, this accuracy should be lower than that achieved by a performer who taps perfectly except for one cue where the tap is $10 \mathrm{~ms}$ early.

A way to neatly meet these criteria is to represent both the stimulus and the taps in indicator vectors $\boldsymbol{x}$ and $\boldsymbol{y}$ with high temporal resolution; that is, $x_{t}=1$ when there is a cue at time $t$, otherwise $x_{t}=0 ; y_{t}=1$ when there is a tap at time $t$, otherwise $y_{t}=0$ (hence the velocity of taps plays no role in this measure, although it could if it were considered useful). We use a temporal granularity of $1 \mathrm{~ms}$; that is, each successive $t$ is $1 \mathrm{~ms}$ after the previous. The two vectors $\boldsymbol{x}$ and $\boldsymbol{y}$ are each circularly convolved by a truncated normal distribution (a kernel). The resulting distributions are mathematically equivalent to adding normally distributed noise to the tap times and the cue times, and serve to approximate inaccuracies of continuous time perception. Indeed, the standard deviation $\sigma$ of the kernel models the probability distribution of possible timings that would be perceived under a time difference limen (just noticeable time difference) of $\sigma$ milliseconds. For these data, we set the standard deviation to $10 \mathrm{~ms}$, which worked well for the visualizations above and which is similar to the $6 \mathrm{~ms}$ just noticeable difference for isochronous sequences (Friberg \& Sundberg, 1995). The similarity of these two vectors is then computed using their cosine similarity: $\frac{\langle\boldsymbol{x}, \boldsymbol{y}\rangle}{\sqrt{\langle\boldsymbol{x}, \boldsymbol{x}\rangle\langle\boldsymbol{y}, \boldsymbol{y}\rangle}}$, where $\langle\boldsymbol{x}, \boldsymbol{y}\rangle$ denotes the inner product of $\boldsymbol{x}$ and $\boldsymbol{y}$. This dependent variable is denoted tap_acc, and is a real number in the unit interval. 


\section{A. 2 Mathematical specification of predictors}

Here, mathematical specifications are provided for the more complex predictors from those outlined in Section 2.2. In the equations below, all indices of the predictors and the rhythm's indicator vectors, are denoted by $n$ taken modulo $N$, which is the number of pulses in the rhythmic period. Cues are indexed by $k$ and when necessary also by $j$, both taken modulo $K$, which is the number of cues in the rhythmic period. We represent a rhythm's indicator function by $\boldsymbol{x}$, which is a binary vector with $N$ entries indexed by periodic pulse number $n \in$ $\{0,1, \ldots, N-1\}$ where 1 represents a cued pulse and 0 represents an uncued pulse.

- balance and evenness: Let us represent a rhythm $\boldsymbol{x}$ with a vector $\boldsymbol{t}(\boldsymbol{x})$ of the times its $K$ cues occur in ascending order and in pulse units; for instance, the rhythm with indicator vector $\boldsymbol{x}=\left(\begin{array}{lllll}1 & 0 & 1 & 0 & 1\end{array}\right)$ has $\boldsymbol{t}(\boldsymbol{x})=\left(\begin{array}{lll}0 & 2 & 4\end{array}\right)$. Balance and evenness are given by

$$
\begin{gathered}
\operatorname{balance}(\boldsymbol{x})=1-\left|\sum_{k=0}^{K-1} \mathrm{e}^{2 \pi \mathrm{i}\left(\boldsymbol{t}(\boldsymbol{x})_{k}\right) / N}\right| / K \\
\operatorname{evenness}(\boldsymbol{x})=\left|\sum_{k=0}^{K-1} \mathrm{e}^{2 \pi \mathrm{i}\left(\left(\boldsymbol{t}(\boldsymbol{x})_{k} / N\right)-(k / K)\right)}\right| / K
\end{gathered}
$$

- IOI_ent: Entropy quantifies the unpredictability of a probability mass function. Normalized entropy is a unit-less form of entropy suitable for comparisons across probability mass functions with differing supports. The normalized entropy of probability mass function $\boldsymbol{x}$ is given by $H(\boldsymbol{x})=-\frac{1}{\log N} \sum_{n=1}^{N} x_{n} \log x_{n}$, where $\log N$ is the maximum possible entropy for a probability mass function with a support of size $N$. If the mass function is dominated by a small number of different outcomes with relatively high probabilities, new outcomes are easier to predict correctly and the entropy is low; if the mass function has a large number of different outcomes with similar probabilities, new outcomes are harder to predict correctly and the entropy is high. To calculate the interonset interval entropy, we calculate the probability mass function of all interonset intervals (between consecutive cues) in one period of the rhythm. For example, in the rhythm (1 $\left.\begin{array}{llll}1 & 1 & 0 & 1\end{array}\right)$, there is one IOI with a 1-pulse duration, and two IOIs with durations of 2 pulses; there are no IOIs of any other duration. Hence, the probability mass function over the two different IOIs is $x=(1 / 3,2 / 3)$. The entropy of this is calculated and then normalized by dividing by $\log 5$ (because there are five possible IOIs in a rhythm with 5 pulses to the period).

- int_ent: Interval entropy is calculated in exactly the same way as IOI_ent except that IOIs between all pairs of cues in the rhythm - not just consecutive cues - are considered.

- $C Q$ : The coherence quotient is one minus the number of coherence failures divided by the maximum number of coherence failures possible for a rhythm with $K$ cues (Carey, 2007). A coherence failure occurs whenever $x_{j+\ell}-x_{j}>x_{k+m}-x_{k}$ (subscripts taken modulo $K$ ) and $\ell<m$, In words, a coherence failure occurs when a pair of cues with a larger generic size (the generic sizes are $\ell$ and $m$ ) than another two cues does not have a greater specific size (the specific sizes are $x_{j+\ell}-x_{j}$ and $x_{k+m}-x_{k}$ ). We would expect rhythms with higher $C Q$ to be easier to tap along with. For rhythms with two cues, the maximum possible number of coherence failures is zero, hence the coherence quotient is undefined. We replace these values with a coherence quotient of 1 ; this is somewhat arbitrary but there are only 9 such rhythms in our set of 91 . 
- $S Q$ : The sameness quotient is one minus the number of different specific sizes taken by all generic intervals $\sum_{m=1}^{K-1}\left|\left\{x_{k+m}-x_{m}: k \in\{0,1, \ldots, K-1\}\right\}\right|$ divided by the maximum of such sizes possible for a pattern with $K$ cues. We would expect rhythms with higher SQ to be easier to tap along with.

- seq_exp: Sequential expectation simulates the expectation that pulse $x_{n}$ is a cue, conditioned on specific timing patterns of prior cues: if there has been a sequence of regular (evenly spaced) cues, the predictor assigns a higher expectation of a cue occurring on the pulse that continues that sequence. However, that expectation is weighted according to that isochronous cue-sequence's length in numbers of cues (denoted $\ell$ ), and the size of its interval onset intervals (denoted $m$ ). These weights are the respective nonlinear parameters $\lambda$ and $\mu$, which are both positive real numbers. Expected parameter values would be greater than 0 , because this implies that cues further back in the sequence are less important, and cues belonging to sequences with longer IOIs are less important.

$$
\operatorname{seq\_ } \exp (\boldsymbol{x} ; \lambda, \mu)_{n}=\sum_{\ell=1}^{L} \sum_{m=1}^{M} \ell^{-\lambda} m^{-\mu} x_{n-\ell m} / \sum_{\ell=1}^{L} \sum_{m=1}^{M} \ell^{-\lambda} m^{-\mu}
$$

For computational feasibility, the upper bounds of the summations are set to values $L$ and $M$ sufficiently large that $L^{-\lambda} M^{-\mu} \approx 0$. In our calculations, we started with $L=27$ and $M=13$, which would be sufficient for $\lambda, \mu>1$ and could be revised upwards if either parameter was estimated close to, or below, 1 (which they were not). The nonlinear parameters $\lambda$ and $\mu$ must be optimized with cue also included in the model, or they will become artificially inflated to boost the $N$ th lag to make up for the missing (and likely important) zero-lag represented by cue (note that lags $0, N, 2 N, 3 N, \ldots$ are equivalent because all indices are taken modulo $N$ ). The parameters were optimized to the tapping data separately from the other predictors; the resulting values are $\lambda=2.2367$ and $\mu=$ 2.1397; hence, the importance of cues in sequences is heavily penalized by their sequence position and the IOI sizes of the sequence.

- $A P M$ : The column sum of the autocorrelation phase matrix is calculated by

$$
\operatorname{APM}(\boldsymbol{x})_{n}=\frac{1}{N} \sum_{\ell=0}^{N-1} \sum_{i=0}^{N-1} x_{\ell i+n} x_{\ell(i+1)+n}
$$

- height: The mean cue height relative to each pulse (inspired by the descriptions of pitch height given in Temperley \& Tan, 2013 but generalized to uncued pulses) is given by

$$
\operatorname{height}(\boldsymbol{x})_{n}=\frac{1}{N} \sum_{m=1-N}^{N-1} m x_{n} x_{n+m}
$$

In this way, the calculation subtracts all periodic cue intervals, from $x_{n}$, that occur before (below) $x_{n}$ from all periodic cue intervals, from $x_{n}$, that occur after (above) $x_{n}$. 
- proj_cent: When all pulses are represented as complex numbers on the unit circle, the projected centroid is the signed projection of the centroid onto the diameter passing through the pulse in question

$$
\begin{gathered}
\operatorname{proj} \_ \text {cent }(\boldsymbol{x})_{n}=|r| \cos \left(\arg (r)-\frac{2 \pi n}{N}\right), \text { where } \\
r=\frac{1}{K} \sum_{n=0}^{N-1} x_{n} \mathrm{e}^{-\frac{2 \pi \mathrm{i} n}{N}}
\end{gathered}
$$

- edge: Due to circularity, we use a discrete kernel $v$ that is the derivative of a zero-mean von Mises distribution, which has a single nonlinear concentration parameter $\kappa$. The value of this parameter, $\kappa=15.678 \mathrm{~ms}$, was chosen by hand to produce results that reliably picked out the edges of groups (note that this corresponds to 6.7 hundredths of a pulse, which are the units used in the numerical calculation):

$$
\begin{aligned}
& \boldsymbol{v}[m ; \kappa]=-\kappa \sin \left(\frac{2 \pi m}{N}\right) \mathrm{e}^{\kappa \cos \left(\frac{2 \pi m}{N}\right) / 2 \pi I_{0}(\kappa)} \\
& \operatorname{edge}(\boldsymbol{x} ; \kappa)_{n}=(|\boldsymbol{x} * \boldsymbol{v}|)_{n}
\end{aligned}
$$

where $*$ denotes circular convolution and $I_{0}$ is the modified Bessel function of the first kind.

- Markov2, 3, 4, ..: For each $S=2,3$ or 4 , we define

$$
\operatorname{Markov} S(\boldsymbol{x})_{n}=\frac{\left|\left\{k \mid\left(x_{k-s}, \ldots, x_{k-1}, x_{k}\right)=\left(x_{n-s}, \ldots, x_{n-1}, 1\right)\right\}\right|}{\left|\left\{k \mid\left(x_{k-s}, \ldots, x_{k-1}\right)=\left(x_{n-s}, \ldots, x_{n-1}\right)\right\}\right|},
$$

which is the cue probability at $n$ as predicted by the maximum-likelihood description of $\boldsymbol{x}$ as an $S$-step Markov chain. 


\section{A. 3 Interpreting the models' coefficients}

The interpretations of the models' coefficients (effects) depend on the modelled distribution of the dependent variable and any link function that is used to map that distribution's mean to a linear combination of the predictors. These are now explained.

The rhythm-level accuracy model has a response variable that is beta-distributed (conditional on the predictors). The mean of the beta distribution is in the unit interval and we used a logit link function to map it to the linear predictor. In comparison to a standard linear model with no link function, this complicates the interpretation of each predictor but, in summary, given a tap accuracy of $y$, a coefficient $\beta_{\mathrm{x}}$ implies that a unit increase in $x$ multiplies $y$ by $\frac{\exp \left(\beta_{\mathrm{x}}\right)}{\left(\exp \left(\beta_{\mathrm{x}}\right)-1\right) y+1}$. Note, therefore, that this multiplicative increase differs according to the starting value of tap accuracy, $y$, and the resulting accuracy value is always in the unit interval.

As outlined earlier, all of the pulse-level models are constructed so that all selected predictors interact with cue. This enables us to determine the independent effect of each predictor on incorrect taps (i.e., probability of tapping within the window of a uncued pulse) and on correct taps (i.e., probability of tapping within the window of a cued pulse), as well as the difference between these two (e.g., how well the taps discriminate between uncued and cued pulses).

The distribution used for the tap_num model is beta-binomial. This is similar to the binomial distribution, but the probability of tapping at each periodic pulse randomly varies according to a beta distribution. It has a wider dispersion than the binomial distribution and fitted the data better. As with binomial, this distribution requires a link function such as logit or probit to transform the linear predictor's values into the unit interval; we used the former link function, which means the coefficients for the predictors, including the interactions with cue, have the meanings that are now explained.

The model can be written with the familiar Wilkinson and Rogers syntax for regression models as tap_num $\sim$ cue $*\left(x_{1}+x_{2}+\cdots\right)$. Using a logit link function, and assuming all continuous variables are centred, the exponentiated value of the intercept, $\exp \left(\beta_{0}\right)$, gives the odds of tapping incorrectly (all predictors at their mean or reference); the exponentiated sum of the intercept and the effect of cue, $\exp \left(\beta_{0}+\beta_{\text {cue }}\right)$, gives the odds for correctly tapping (all other predictors at their mean or reference); $\exp \left(\beta_{\mathrm{x}}\right)$ gives the odds ratio - the multiplicative change in the odds - of incorrectly tapping given a unit increase in $x$ (all else being equal); the exponentiated effect of $\exp \left(\beta_{\mathrm{x}}+\beta_{\text {cue: } \mathrm{x}}\right)$ gives the odds ratio for correctly tapping given a unit increase in $x$ (all else being equal).

There are also useful interpretations in terms of the signal detection theory concept of discriminability (also known as $d^{\prime}$ or $d$-prime) (Milne \& Herff, 2020). This is a standard quantification of how well a "signal" (here, a cue) can be "detected" (here, tapped) in the presence of noise or uncertainty. Under the assumption of uncertainty with a given standard deviation, discriminability is simply the number of standard deviations between no signal (an uncued pulse) and signal (a cued pulse) - the further apart they are, the more easily they can be discriminated (correct taps can be made). When all other predictors are at their mean or reference, $\beta_{\text {cue }} / 1.7$ closely corresponds to the discriminability of cues (as evidenced by tapping); while $\beta_{\text {cue:x }} / 1.7$ closely corresponds to the change in discriminability predicted by a unit increase in $x$ (all else being equal). This means that the effects for the interactions with cue immediately indicate the importance of the associated predictor for facilitating or hindering their discriminability.

Furthermore, $\beta_{0} / 1.7$ closely corresponds to the tapping bias, which is the propensity to not tap, regardless of whether or not there is a cue. Analogously, $\beta_{x}$ gives the amount by which the bias is predicted to change given a unit increase in $x$. (There are alternative 
definitions for bias but this is a common one, Milne \& Herff, 2020.) This is also a useful quantity because it characterizes the extent to which any predictor influences the overall propensity to tap. (Note that if a probit link is used, none of the divisions by 1.7 are necessary and the coefficients correspond exactly to bias, discriminability, and their effects thereon; we use the logit link because the interpretation in terms of odds ratios is probably more interpretable.)

The models for tap_vel and tap_delta have the same structure as that for tap_num; that is, tap_vel $\sim$ cue $*\left(x_{1}+x_{2}+\cdots\right)$ and tap_delta $\sim$ cue $*\left(x_{1}+x_{2}+\cdots\right)$. However, in both cases the dependent variable is treated as normally distributed (conditional on the predictors) and no link function is required. This means the intercept, $\beta_{0}$, gives the predicted tap velocity or timing error for incorrect taps (when all predictors are at their mean or reference). The sum of the intercept and the effect of cue, $\beta_{0}+\beta_{\text {cue }}$ gives the mean tap velocity or timing error for correct taps (when all predictors are at their mean or reference). The coefficient $\beta_{x}$ gives the predicted change in incorrect tap velocity or timing error. Sums of coefficients of the form $\beta_{\text {cue }}+\beta_{\text {cue:x }}$ give the predicted change in correct tap velocity or timing error. 


\section{A. 4 Rhythms tested}

Table 8 . The 91 rhythms used in the experiment: $m$ is the number of long IOIs; $n$ is number of short IOIs ; $K$ is the number of cues; $N$ is the number of pulses; $L$ is length of the long IOIs (in pulses); $s$ is length of the short IOIs (in pulses); $R$ is ratio $L / s$.

\begin{tabular}{ccccccc}
\hline$m$ & $n$ & $L$ & $S$ & $K$ & $N$ & $R$ \\
\hline 1 & 1 & 2 & 1 & 2 & 3 & $2 / 1$ \\
1 & 1 & 3 & 1 & 2 & 4 & $3 / 1$ \\
1 & 2 & 2 & 1 & 3 & 4 & $2 / 1$ \\
1 & 1 & 4 & 1 & 2 & 5 & $4 / 1$ \\
1 & 1 & 3 & 2 & 2 & 5 & $3 / 2$ \\
1 & 2 & 3 & 1 & 3 & 5 & $3 / 1$ \\
2 & 1 & 2 & 1 & 3 & 5 & $2 / 1$ \\
1 & 3 & 2 & 1 & 4 & 5 & $2 / 1$ \\
1 & 1 & 5 & 1 & 2 & 6 & $5 / 1$ \\
1 & 2 & 4 & 1 & 3 & 6 & $4 / 1$ \\
1 & 3 & 3 & 1 & 4 & 6 & $3 / 1$ \\
1 & 4 & 2 & 1 & 5 & 6 & $2 / 1$ \\
1 & 1 & 5 & 2 & 2 & 7 & $5 / 2$ \\
1 & 1 & 4 & 3 & 2 & 7 & $4 / 3$ \\
1 & 2 & 5 & 1 & 3 & 7 & $5 / 1$ \\
2 & 1 & 3 & 1 & 3 & 7 & $3 / 1$ \\
1 & 2 & 3 & 2 & 3 & 7 & $3 / 2$ \\
1 & 3 & 4 & 1 & 4 & 7 & $4 / 1$ \\
3 & 1 & 2 & 1 & 4 & 7 & $2 / 1$ \\
1 & 4 & 3 & 1 & 5 & 7 & $3 / 1$ \\
2 & 3 & 2 & 1 & 5 & 7 & $2 / 1$ \\
1 & 5 & 2 & 1 & 6 & 7 & $2 / 1$ \\
1 & 1 & 5 & 3 & 2 & 8 & $5 / 3$ \\
2 & 1 & 3 & 2 & 3 & 8 & $3 / 2$ \\
1 & 3 & 5 & 1 & 4 & 8 & $5 / 1$ \\
1 & 4 & 4 & 1 & 5 & 8 & $4 / 1$ \\
3 & 2 & 2 & 1 & 5 & 8 & $2 / 1$ \\
1 & 5 & 3 & 1 & 6 & 8 & $3 / 1$ \\
1 & 6 & 2 & 1 & 7 & 8 & $2 / 1$ \\
1 & 1 & 5 & 4 & 2 & 9 & $5 / 4$ \\
2 & 1 & 4 & 1 & 3 & 9 & $4 / 1$ \\
1 & 2 & 5 & 2 & 3 & 9 & $5 / 2$ \\
1 & 3 & 3 & 2 & 4 & 9 & $3 / 2$ \\
1 & 4 & 5 & 1 & 5 & 9 & $5 / 1$ \\
2 & 3 & 3 & 1 & 5 & 9 & $3 / 1$ \\
4 & 1 & 2 & 1 & 5 & 9 & $2 / 1$ \\
1 & 5 & 4 & 1 & 6 & 9 & $4 / 1$ \\
1 & 6 & 3 & 1 & 7 & 9 & $3 / 1$ \\
2 & 5 & 2 & 1 & 7 & 9 & $2 / 1$ \\
1 & 7 & 2 & 1 & 8 & 9 & $2 / 1$ \\
1 & 2 & 4 & 3 & 3 & 10 & $4 / 3$ \\
3 & 1 & 3 & 1 & 4 & 10 & $3 / 1$ \\
1 & 5 & 5 & 1 & 6 & 10 & $5 / 1$ \\
1 & 6 & 4 & 1 & 7 & 10 & $4 / 1$ \\
3 & 4 & 2 & 1 & 7 & 10 & $2 / 1$ \\
1 & 7 & 3 & 1 & 8 & 10 & $3 / 1$ \\
1 & 8 & 2 & 1 & 9 & 10 & $2 / 1$ \\
2 & 1 & 5 & 1 & 3 & 11 & $5 / 1$ \\
1 & 2 & 5 & 3 & 3 & 11 & $5 / 3$ \\
2 & 1 & 4 & 3 & 3 & 11 & $4 / 3$ \\
1 & 3 & 5 & 2 & 4 & 11 & $5 / 2$ \\
3 & 1 & 3 & 2 & 4 & 11 & $3 / 2$ \\
2 & 3 & 4 & 1 & 5 & 11 & $4 / 1$ \\
3 & 2 & 3 & 1 & 5 & 11 & $3 / 1$ \\
& & & & & &
\end{tabular}




\begin{tabular}{lllllll}
1 & 4 & 3 & 2 & 5 & 11 & $3 / 2$ \\
5 & 1 & 2 & 1 & 6 & 11 & $2 / 1$ \\
1 & 6 & 5 & 1 & 7 & 11 & $5 / 1$ \\
2 & 5 & 3 & 1 & 7 & 11 & $3 / 1$ \\
4 & 3 & 2 & 1 & 7 & 11 & $2 / 1$ \\
1 & 7 & 4 & 1 & 8 & 11 & $4 / 1$ \\
3 & 5 & 2 & 1 & 8 & 11 & $2 / 1$ \\
1 & 8 & 3 & 1 & 9 & 11 & $3 / 1$ \\
2 & 7 & 2 & 1 & 9 & 11 & $2 / 1$ \\
1 & 9 & 2 & 1 & 10 & 11 & $2 / 1$ \\
2 & 1 & 5 & 2 & 3 & 12 & $5 / 2$ \\
2 & 3 & 3 & 2 & 5 & 12 & $3 / 2$ \\
5 & 2 & 2 & 1 & 7 & 12 & $2 / 1$ \\
1 & 7 & 5 & 1 & 8 & 12 & $5 / 1$ \\
1 & 8 & 4 & 1 & 9 & 12 & $4 / 1$ \\
1 & 9 & 3 & 1 & 10 & 12 & $3 / 1$ \\
1 & 10 & 2 & 1 & 11 & 12 & $2 / 1$ \\
2 & 1 & 5 & 3 & 3 & 13 & $5 / 3$ \\
1 & 2 & 5 & 4 & 3 & 13 & $5 / 4$ \\
3 & 1 & 4 & 1 & 4 & 13 & $4 / 1$ \\
1 & 3 & 4 & 3 & 4 & 13 & $4 / 3$ \\
2 & 3 & 5 & 1 & 5 & 13 & $5 / 1$ \\
4 & 1 & 3 & 1 & 5 & 13 & $3 / 1$ \\
1 & 4 & 5 & 2 & 5 & 13 & $5 / 2$ \\
3 & 2 & 3 & 2 & 5 & 13 & $3 / 2$ \\
1 & 5 & 3 & 2 & 6 & 13 & $3 / 2$ \\
2 & 5 & 4 & 1 & 7 & 13 & $4 / 1$ \\
3 & 4 & 3 & 1 & 7 & 13 & $3 / 1$ \\
6 & 1 & 2 & 1 & 7 & 13 & $2 / 1$ \\
5 & 3 & 2 & 1 & 8 & 13 & $2 / 1$ \\
1 & 8 & 5 & 1 & 9 & 13 & $5 / 1$ \\
2 & 7 & 3 & 1 & 9 & 13 & $3 / 1$ \\
4 & 5 & 2 & 1 & 9 & 13 & $2 / 1$ \\
1 & 9 & 4 & 1 & 10 & 13 & $4 / 1$ \\
3 & 7 & 2 & 1 & 10 & 13 & $2 / 1$ \\
1 & 10 & 3 & 1 & 11 & 13 & $3 / 1$ \\
2 & 9 & 2 & 1 & 11 & 13 & $2 / 1$ \\
\hline & & & & & & \\
& & &
\end{tabular}




\section{A. 5 Circular descriptive plot calculations}

The circular descriptive plots (Figures 3 and 4) were created with the following method. The tap-data is expressed as a vector $\boldsymbol{y}=\left(y_{1}, y_{2}, \ldots\right)$, whose elements are indexed by $t$ milliseconds and whose entries are the normalized tap velocity (MIDI velocity values are integers from 0 to 127; these were normalized by dividing by 127; a value of 0 corresponds to no tap). For each 30-second performance, the vectors were divided into segments of the length of the rhythmic period, which is $234 \mathrm{Nms}$. The different segments were summed and multiplied by $N$ to account for the differing numbers of repetitions of rhythms with differing $N$ over the 30 -second performance. For each rhythm, these $234 N$-entry vectors were aligned, and then averaged across all performances (remember that for each presentation, rhythms are started with a random phase, which makes it possible to average across them to gain an understanding of how tap velocities and timings vary regardless of start-phase). This results in the distribution of tap velocities over the rhythmic period; in order to approximate the underlying shape of this distribution and make it easier to see, the data is smoothed by convolution with a truncated discrete normal distribution with a standard deviation of $10 \mathrm{~ms}$. 
A. 6 Smoothed velocity distributions for all 91 rhythms

Solid radii mark cued pulses; dashed radii mark uncued pulses. The data are normalized for each rhythm.
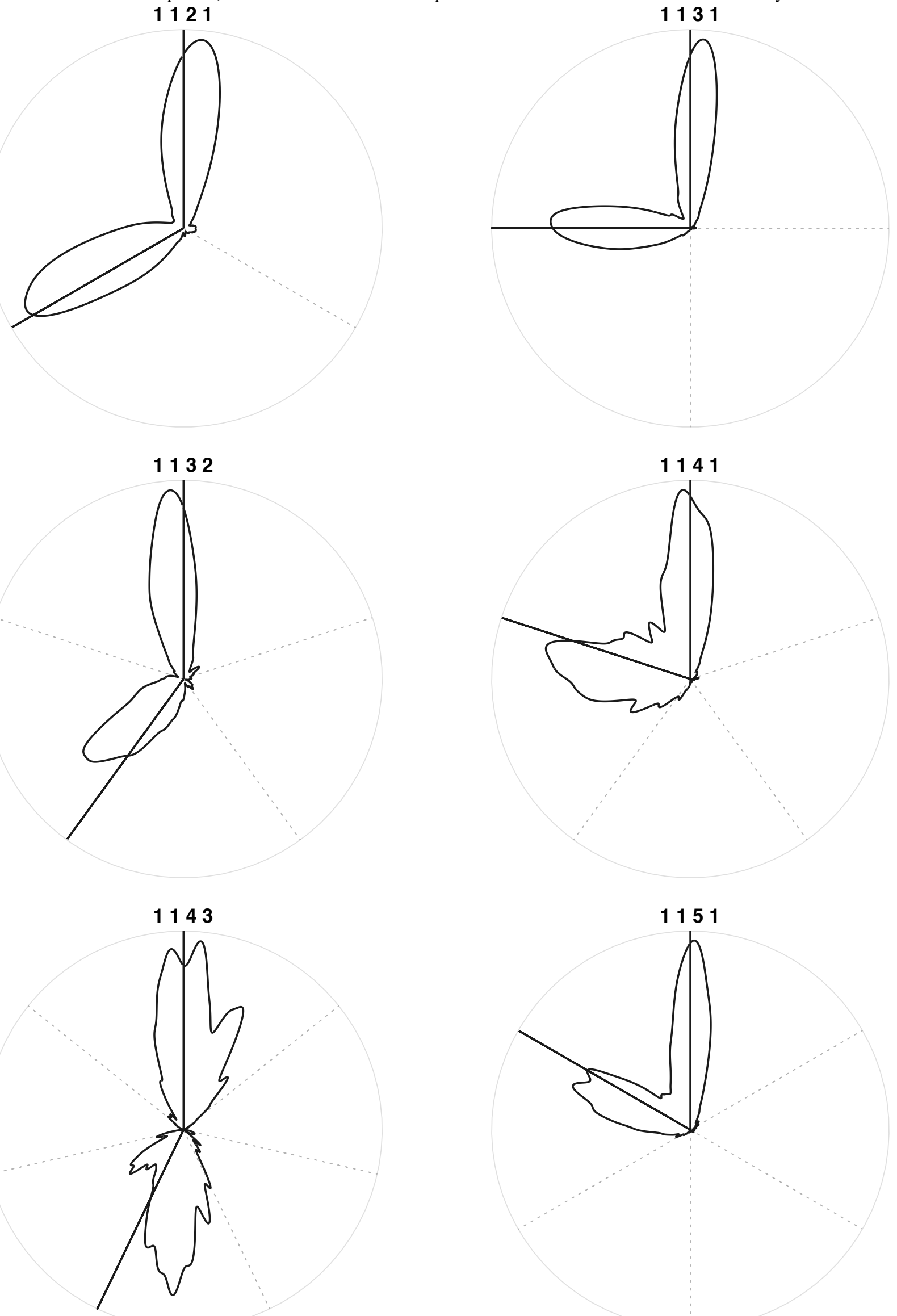

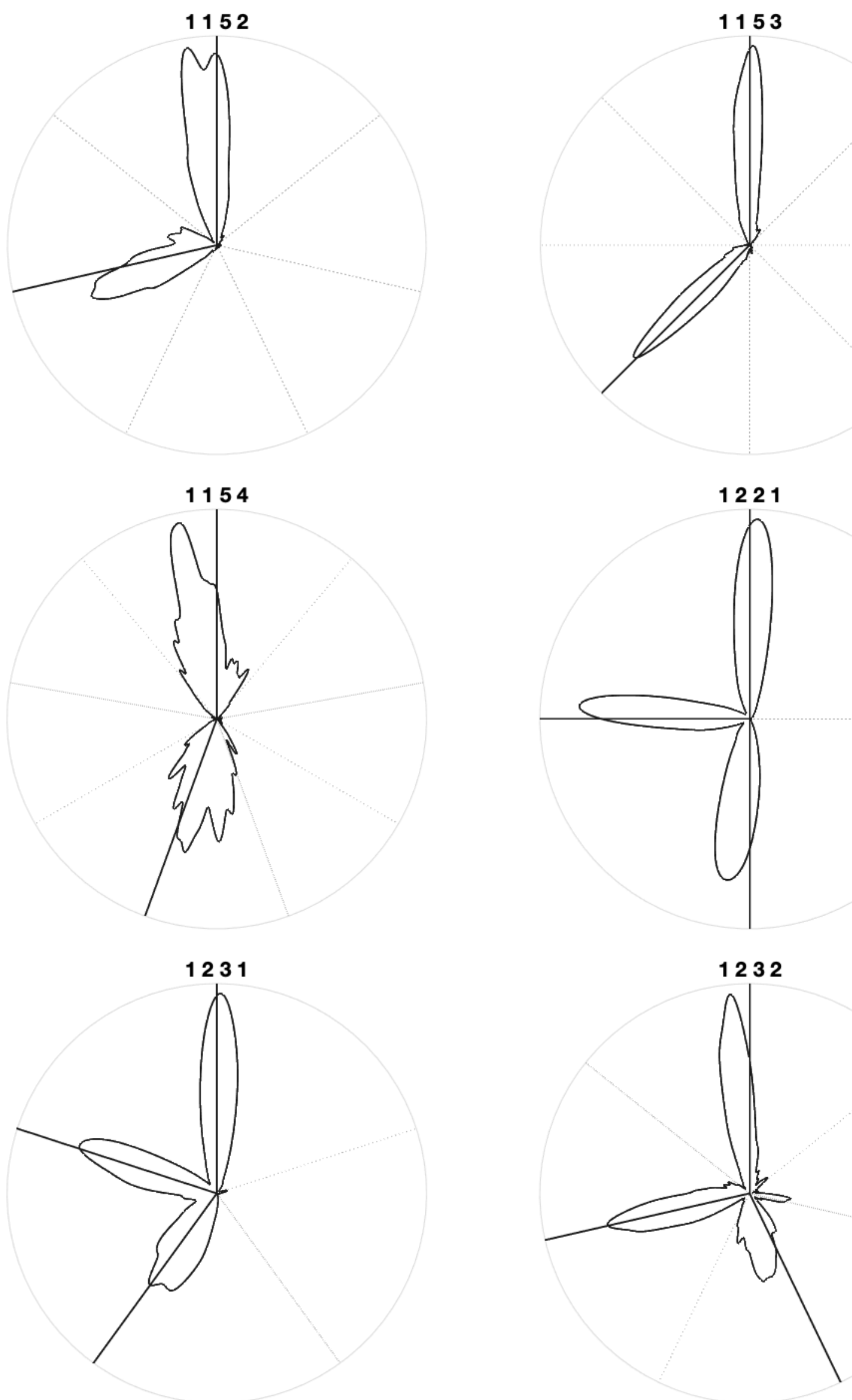

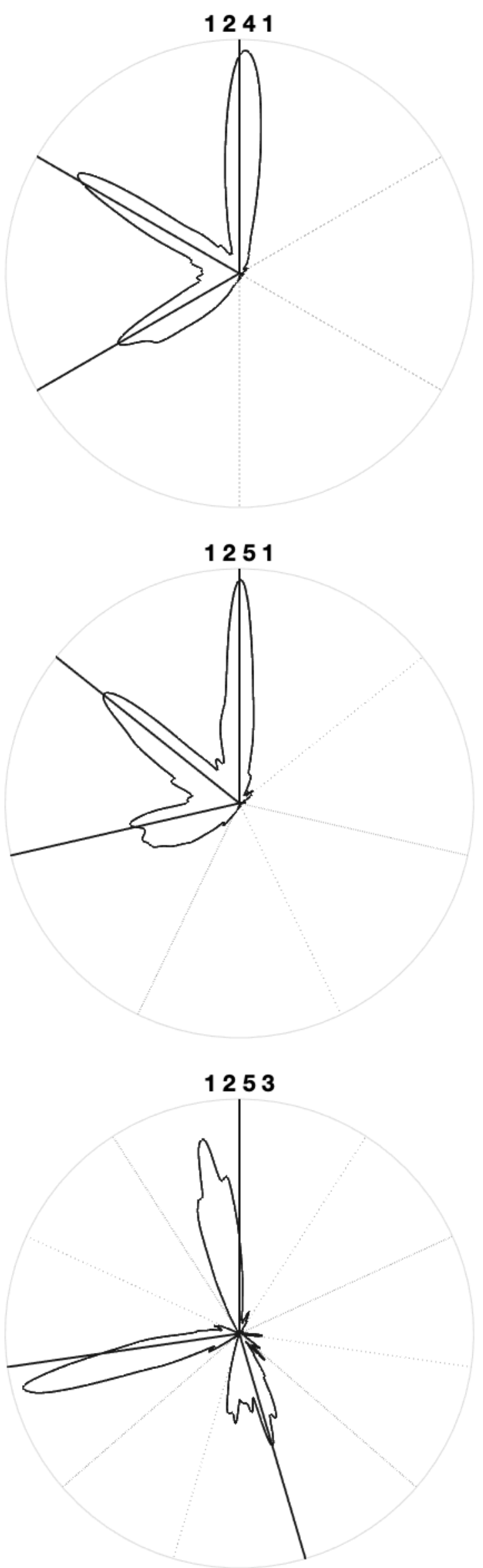
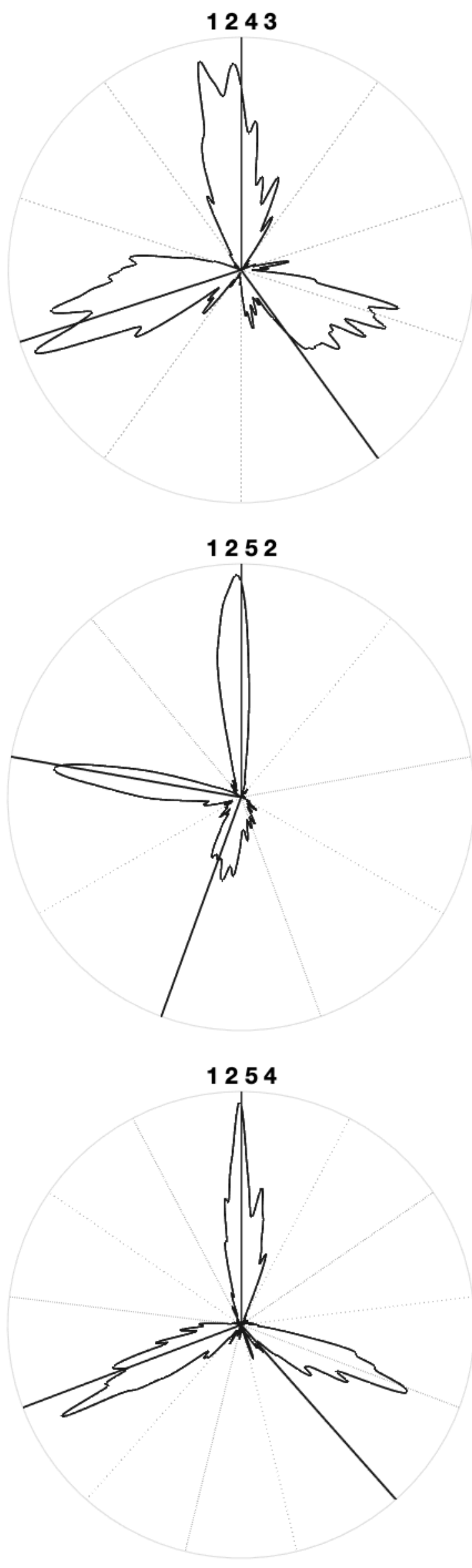

50 

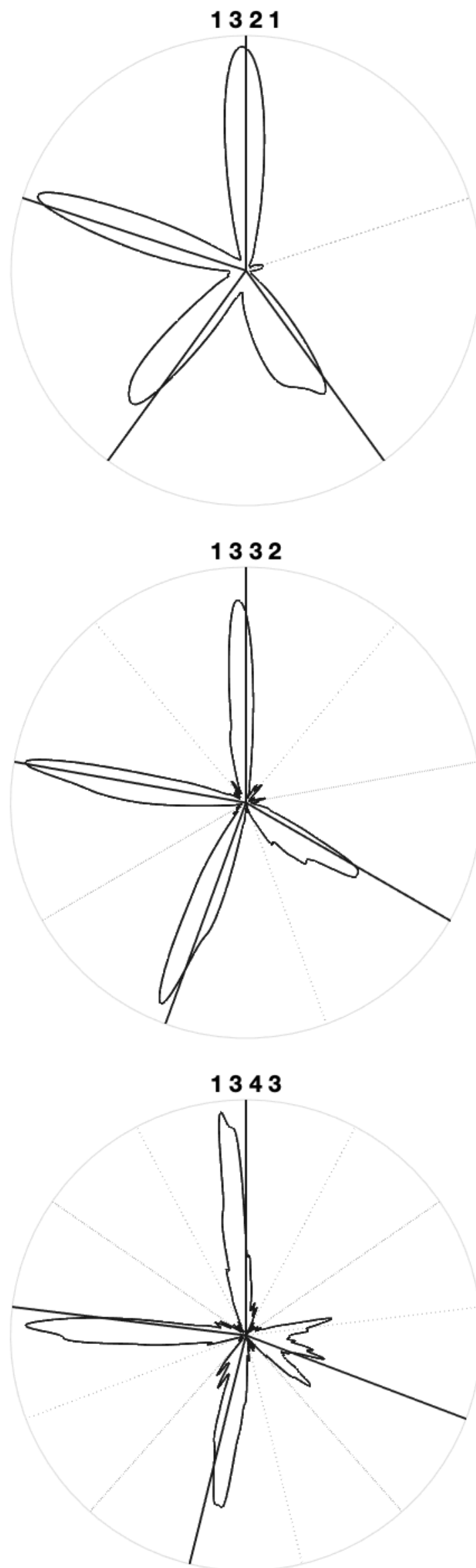
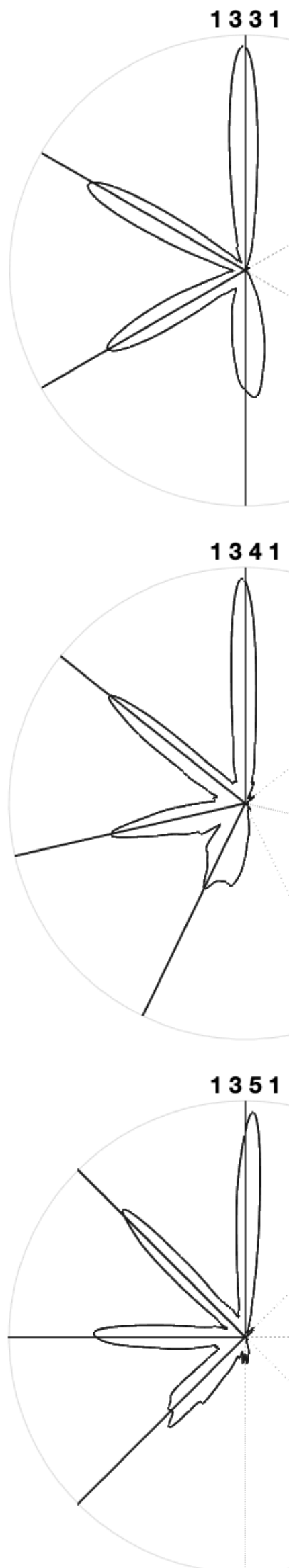

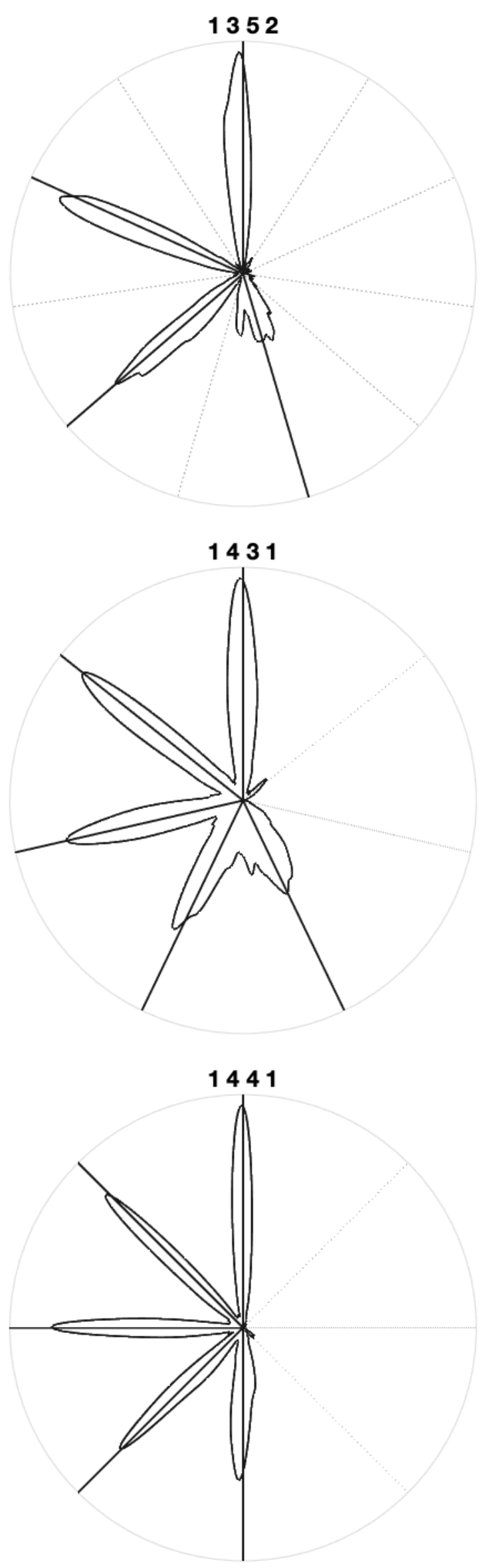
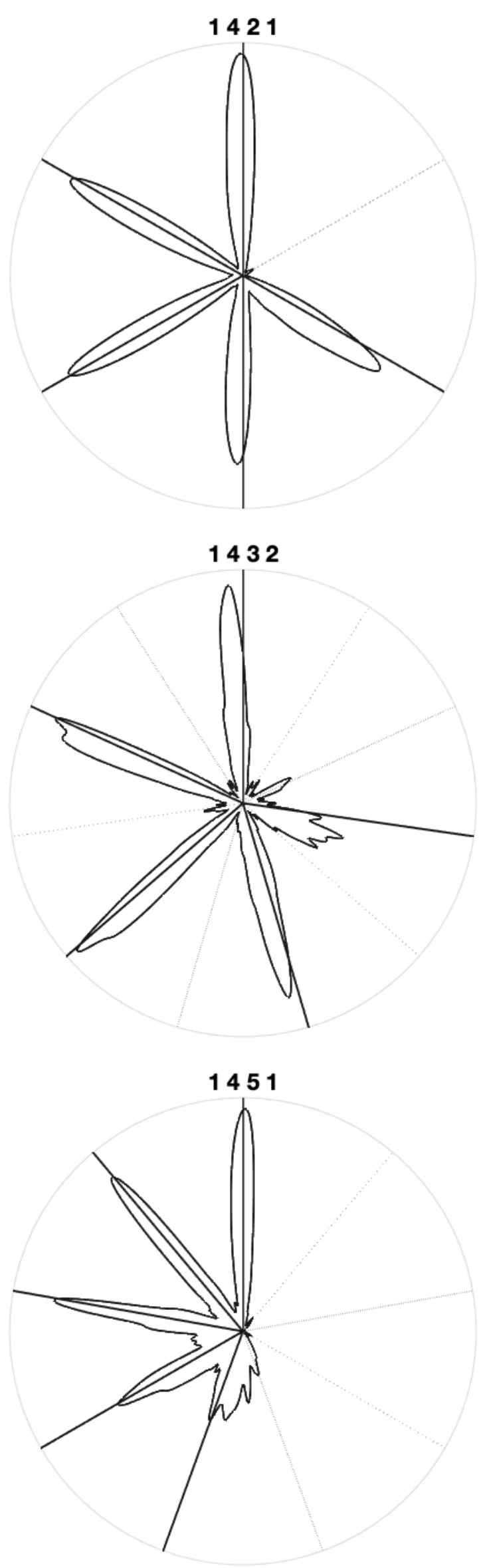

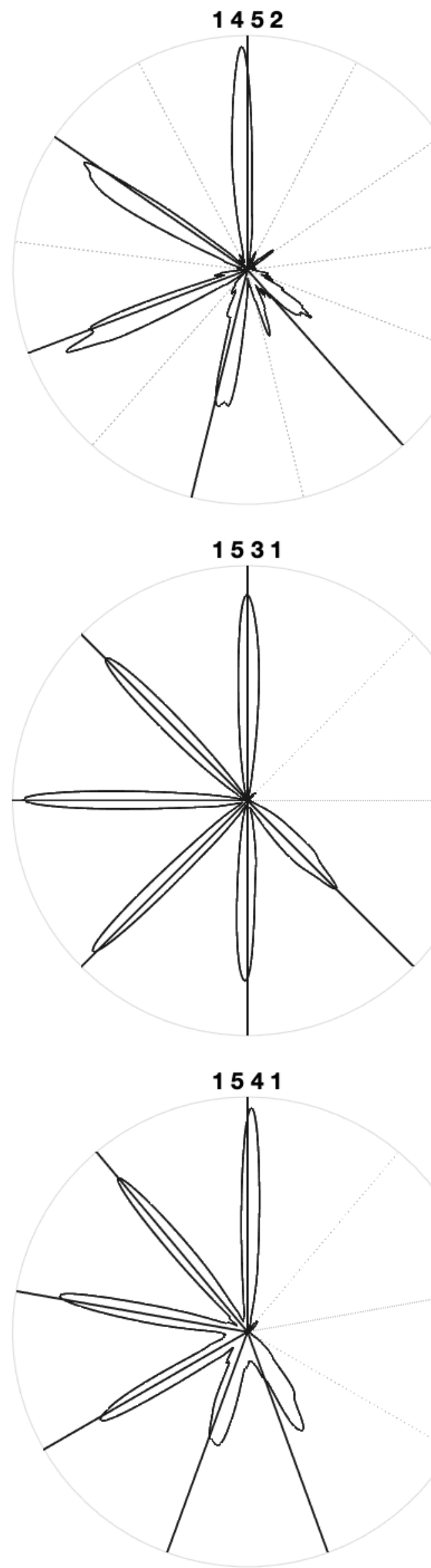
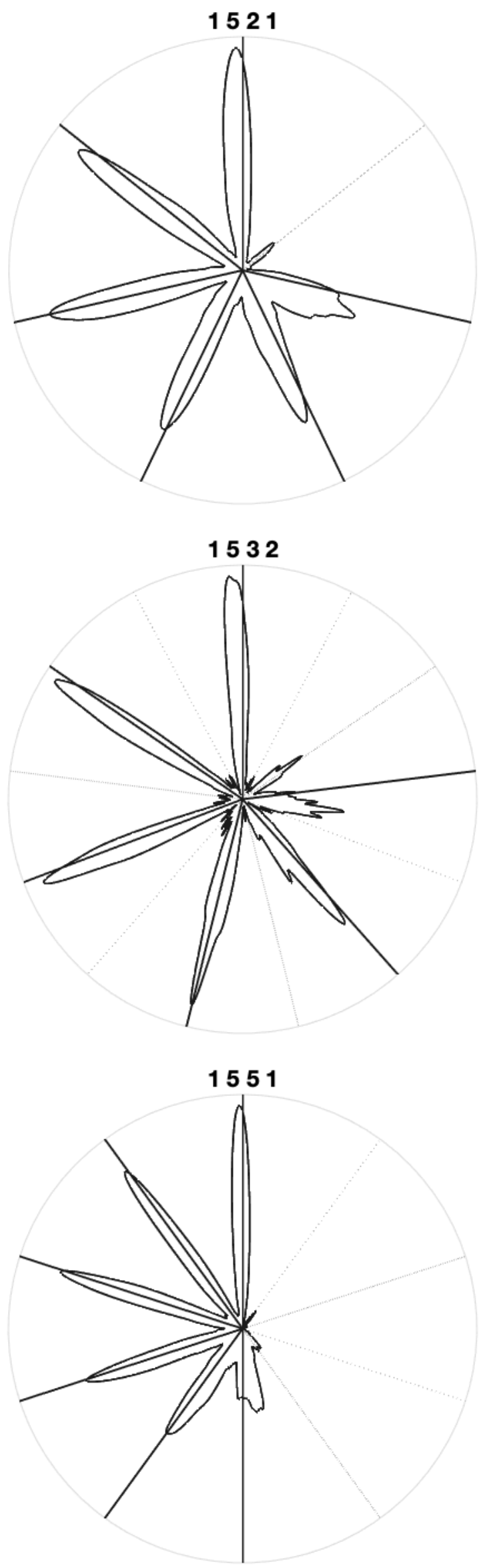

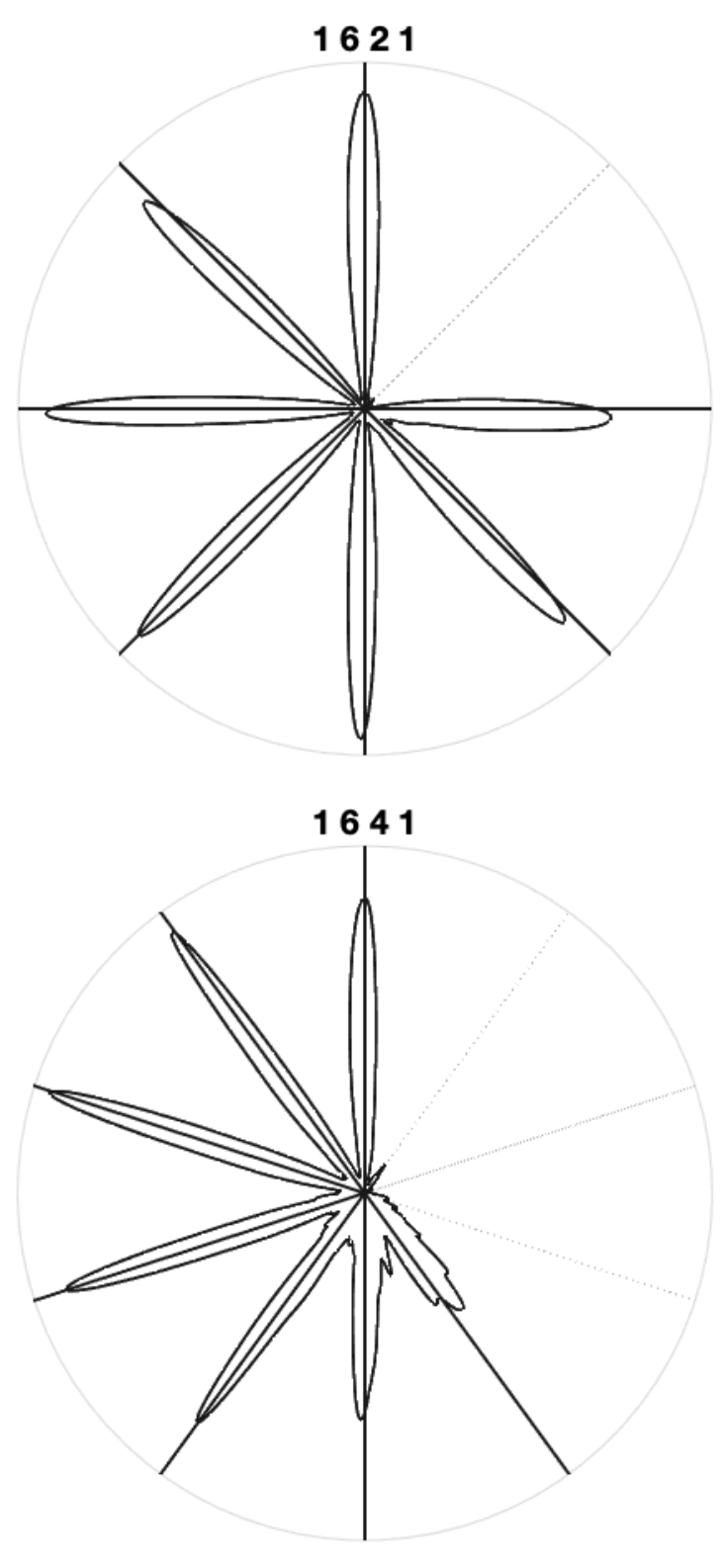

1721

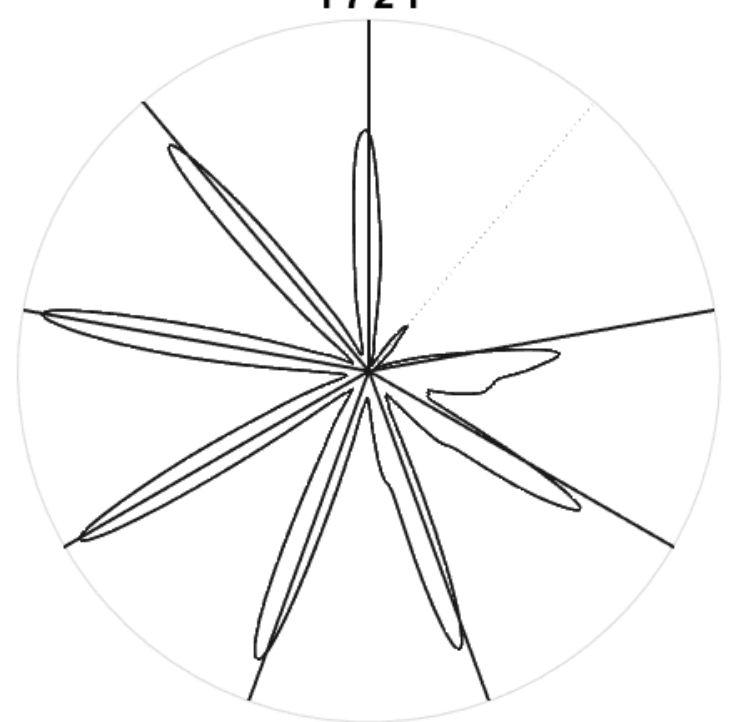

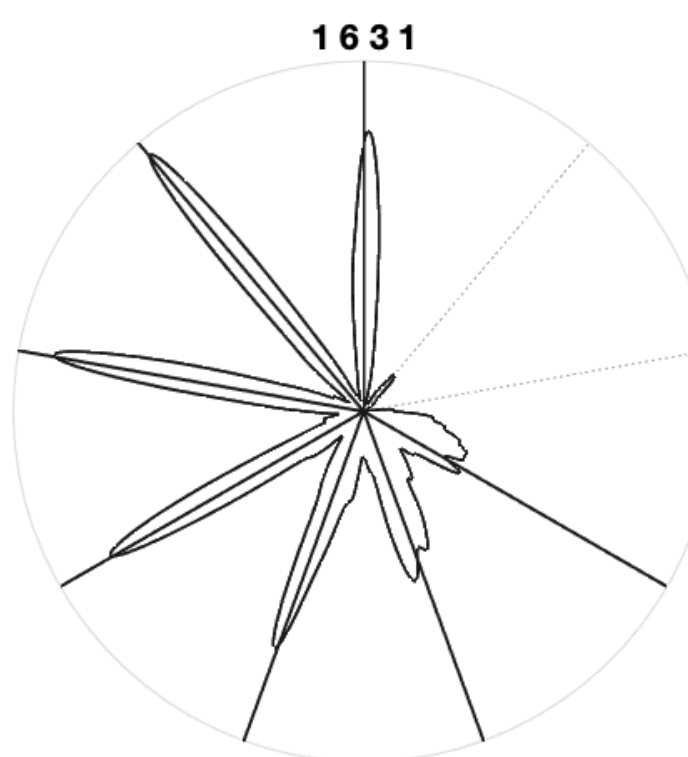

1651

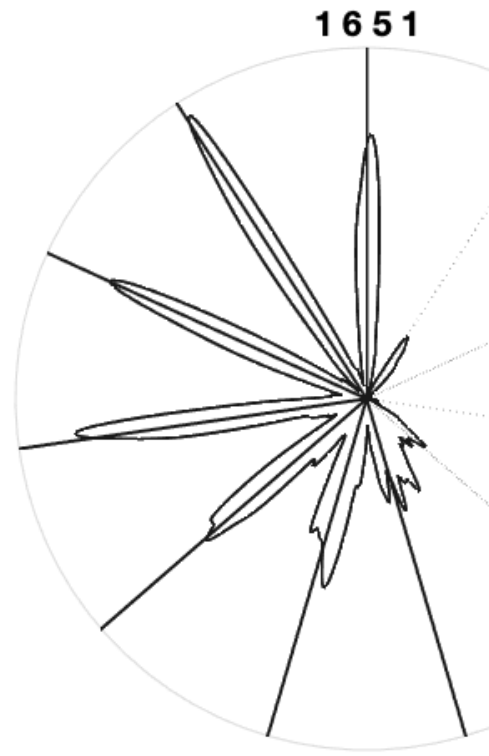

1731

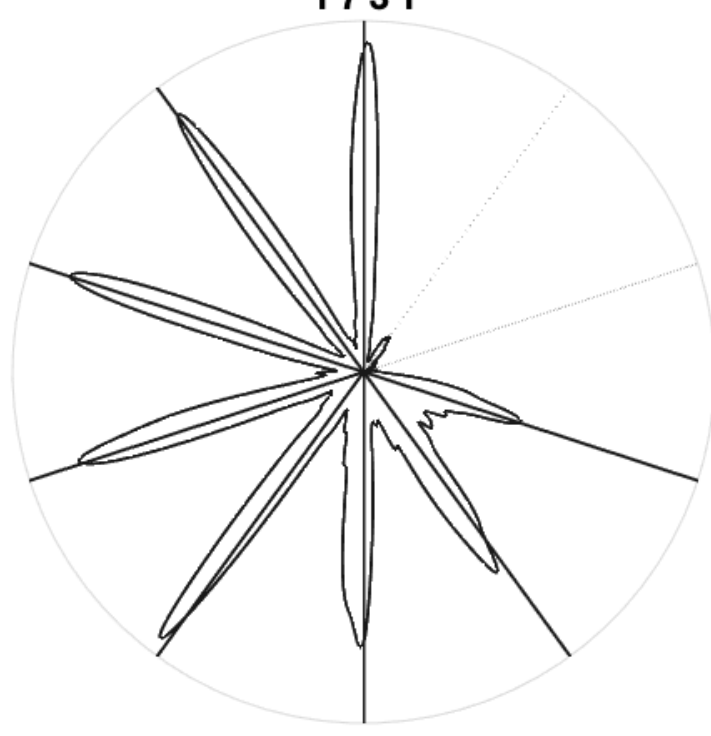



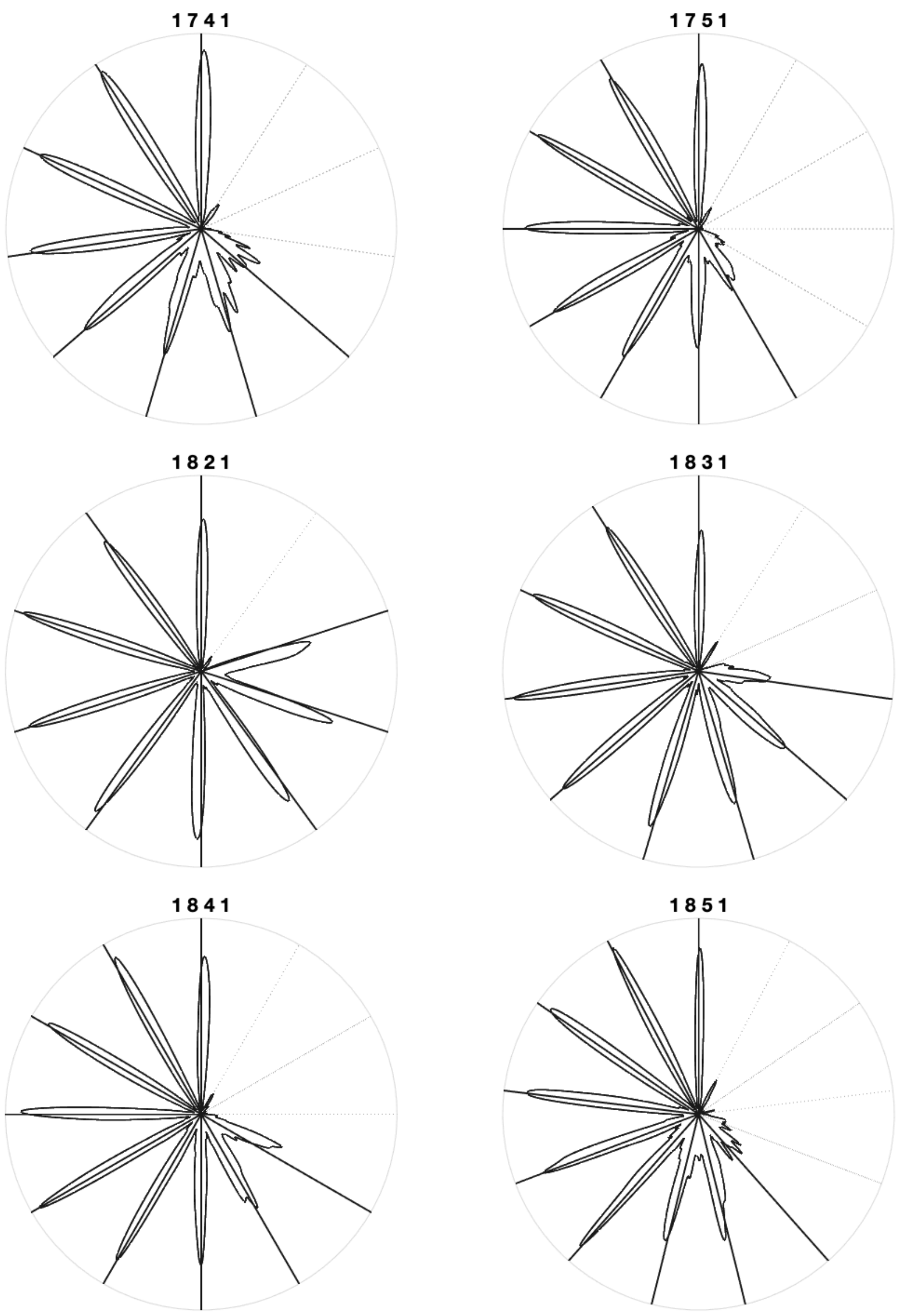

55 

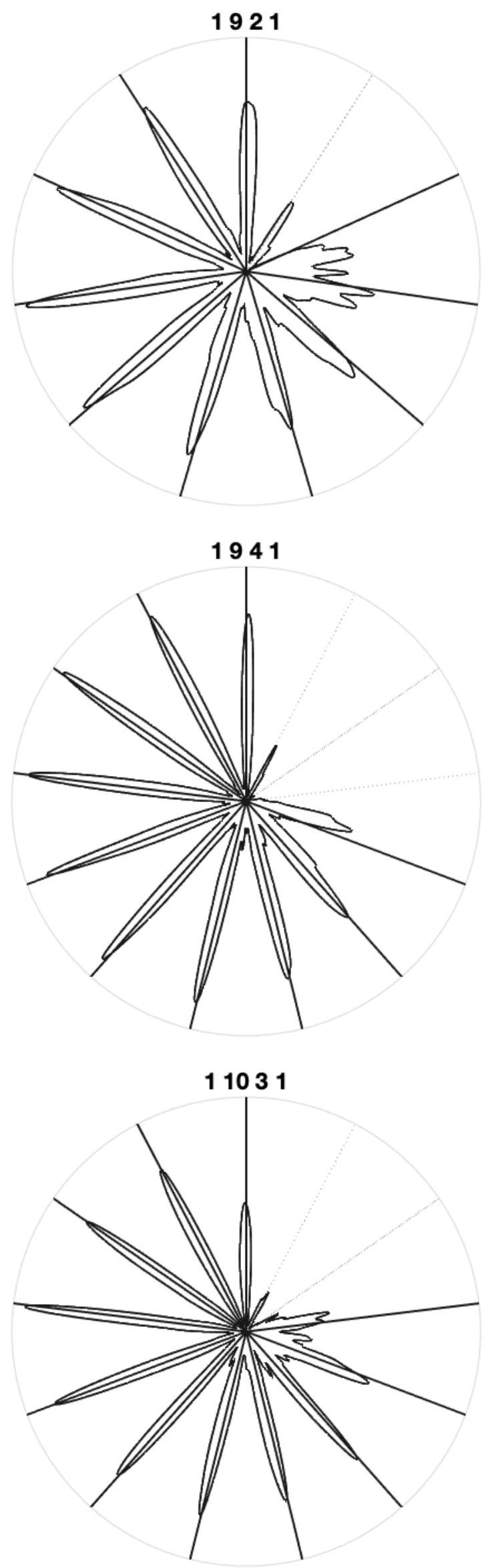
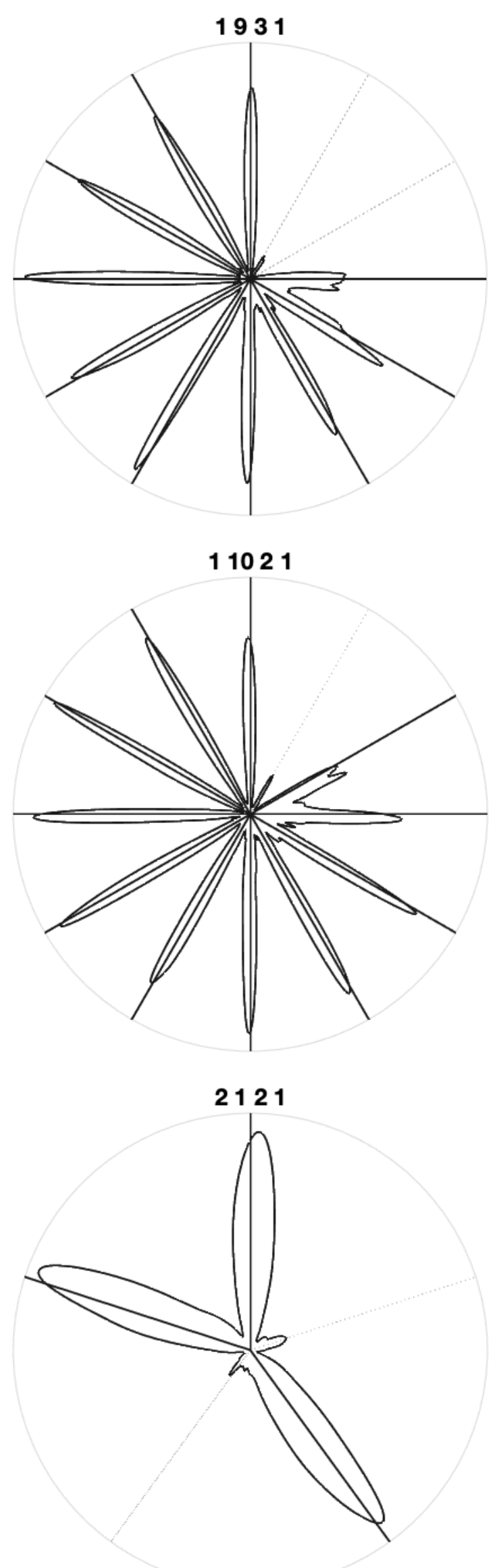

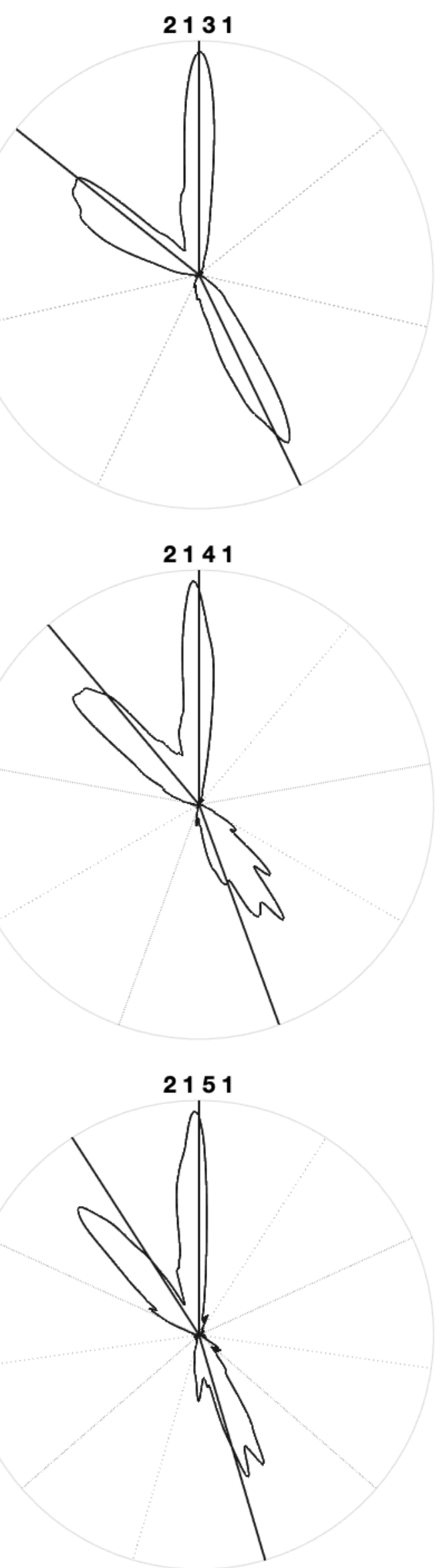
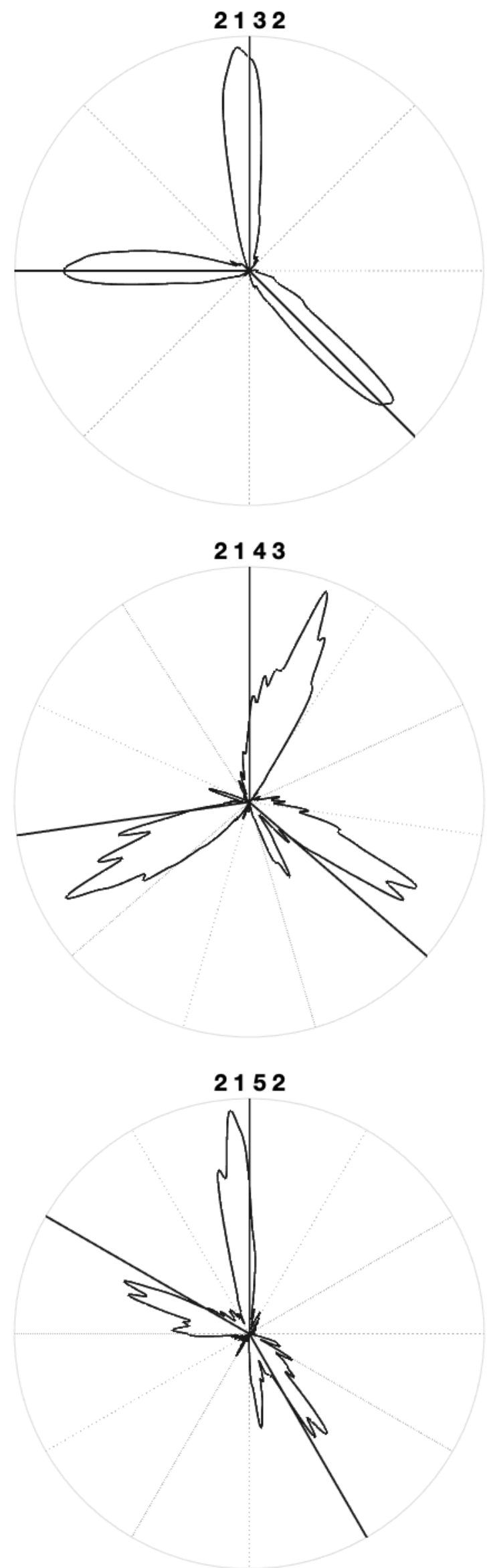

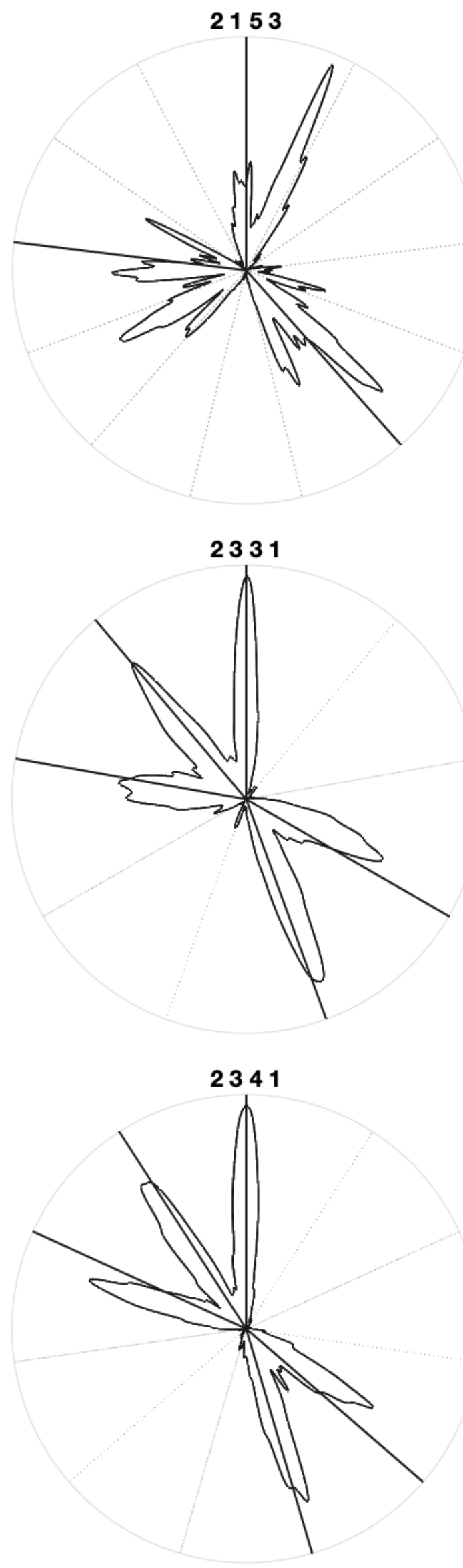
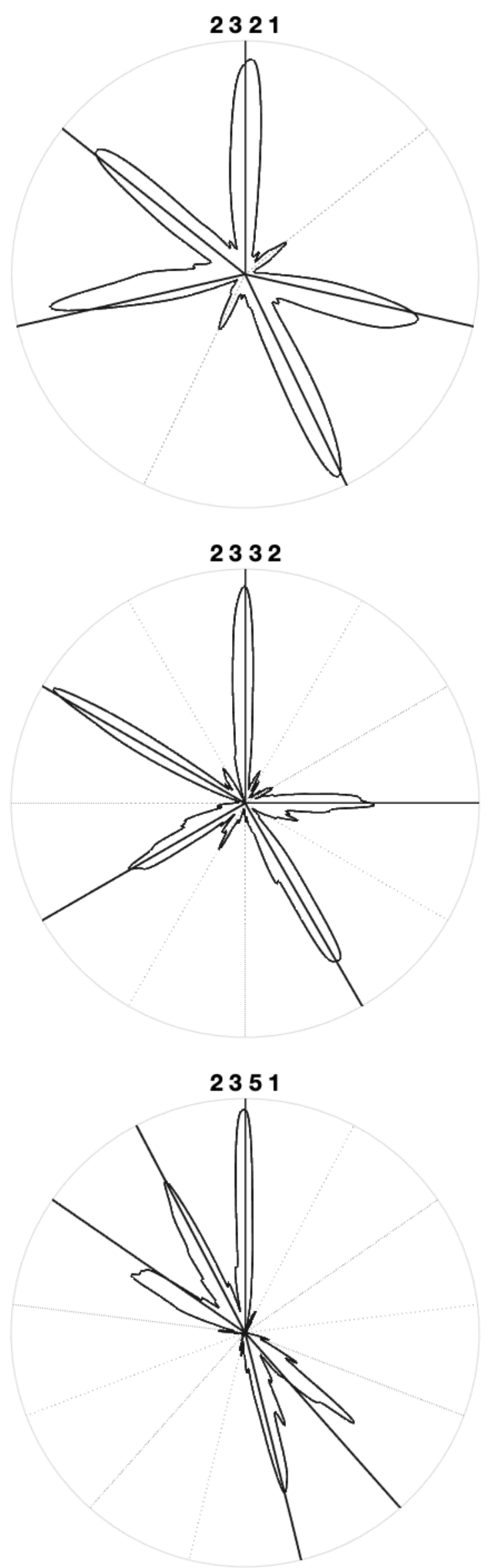

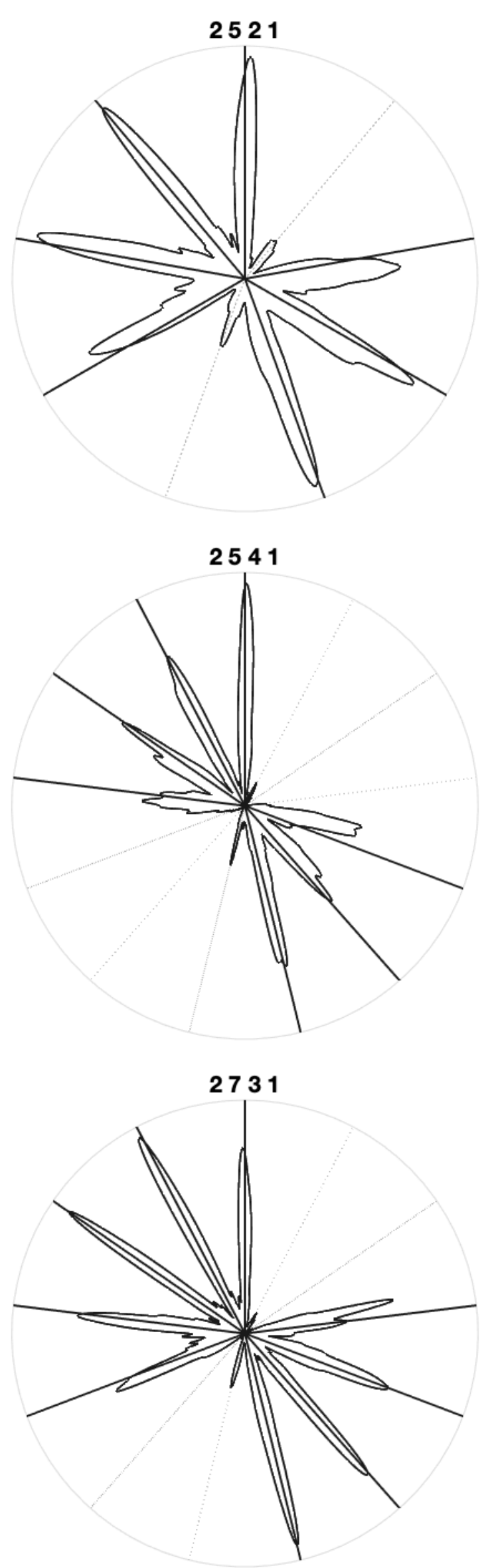
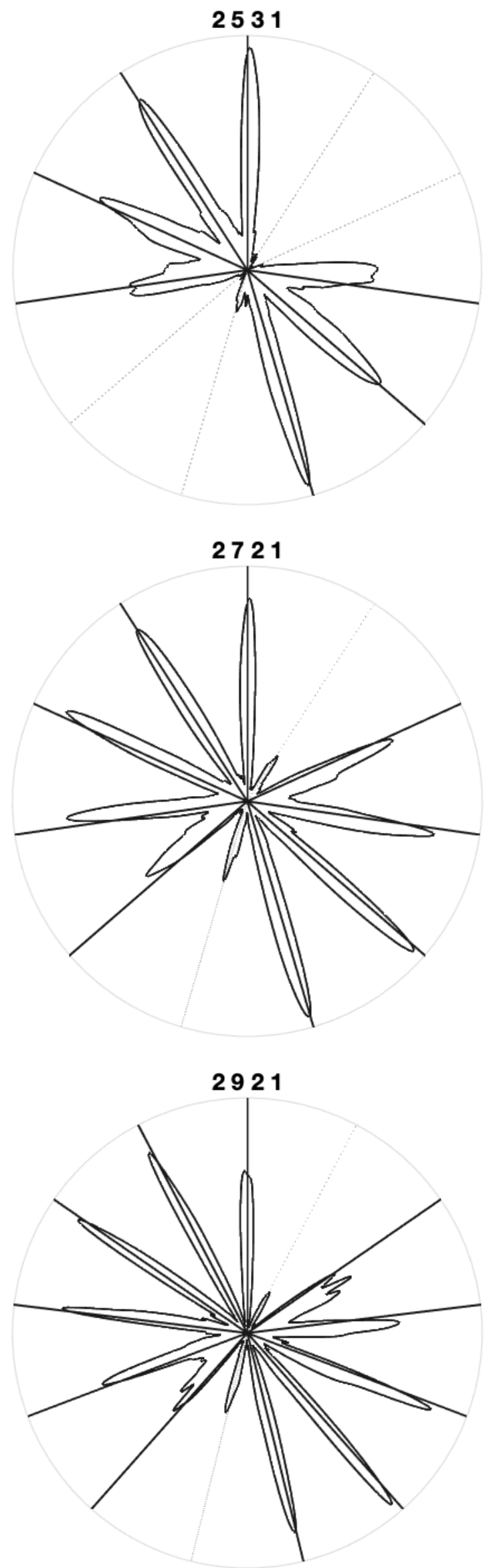

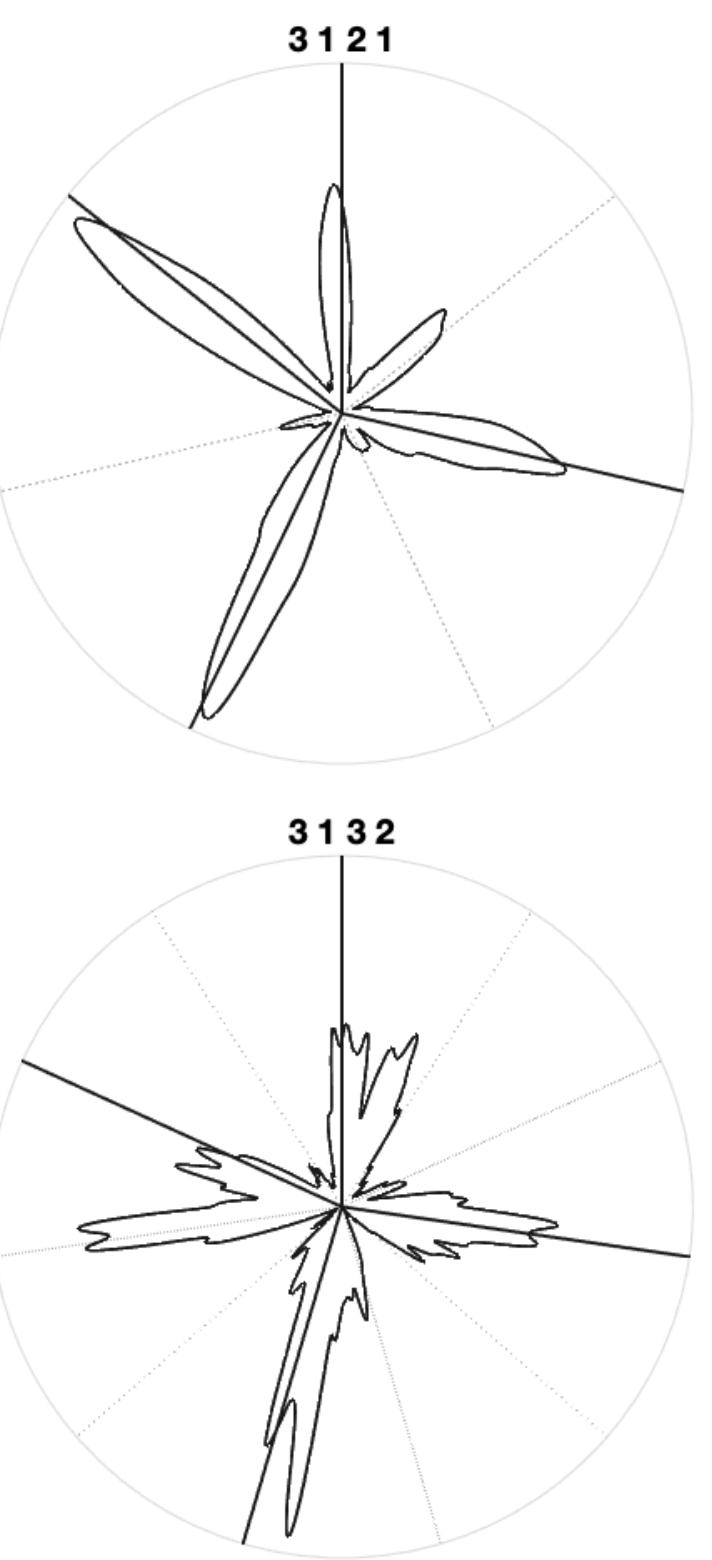

3221

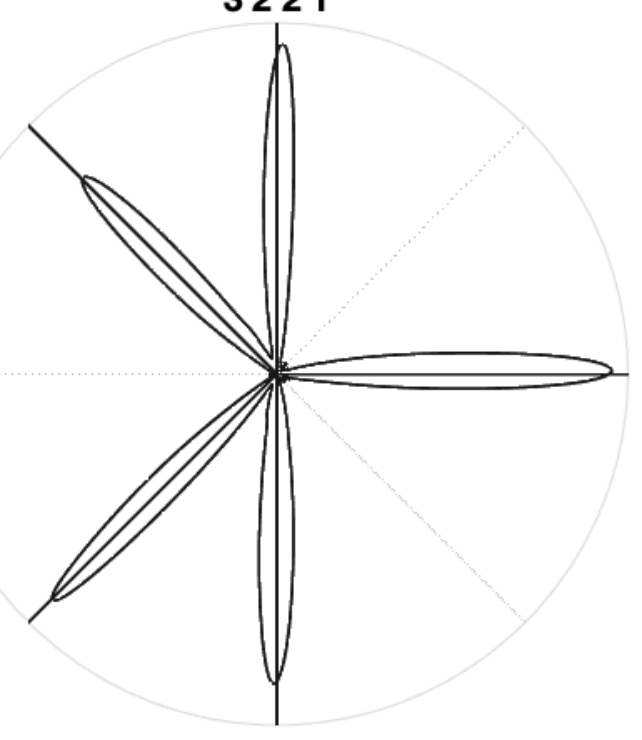

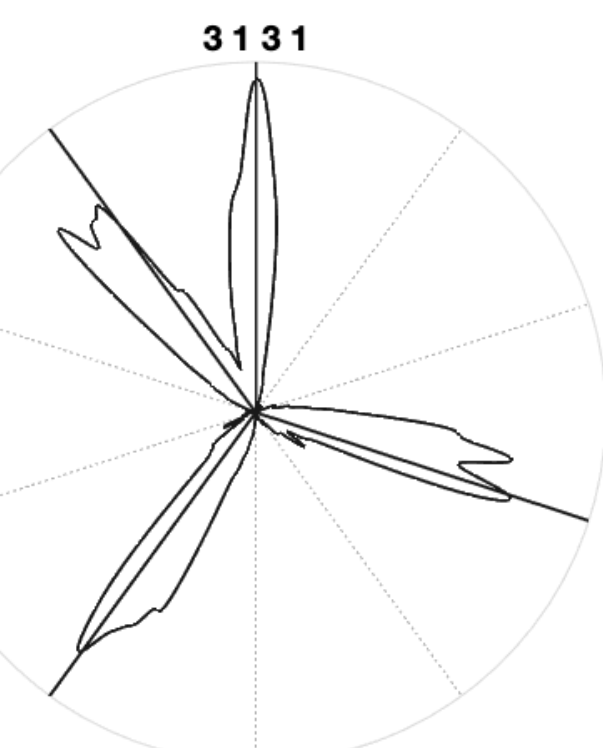
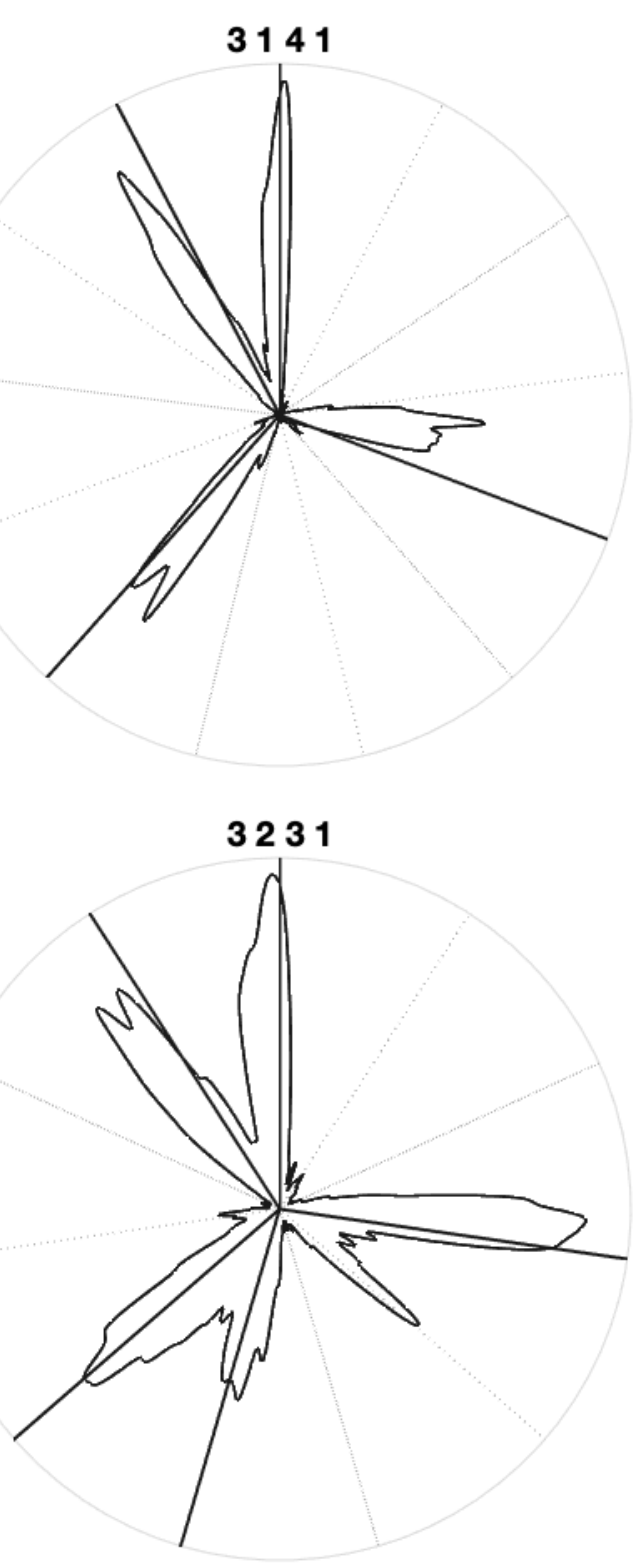

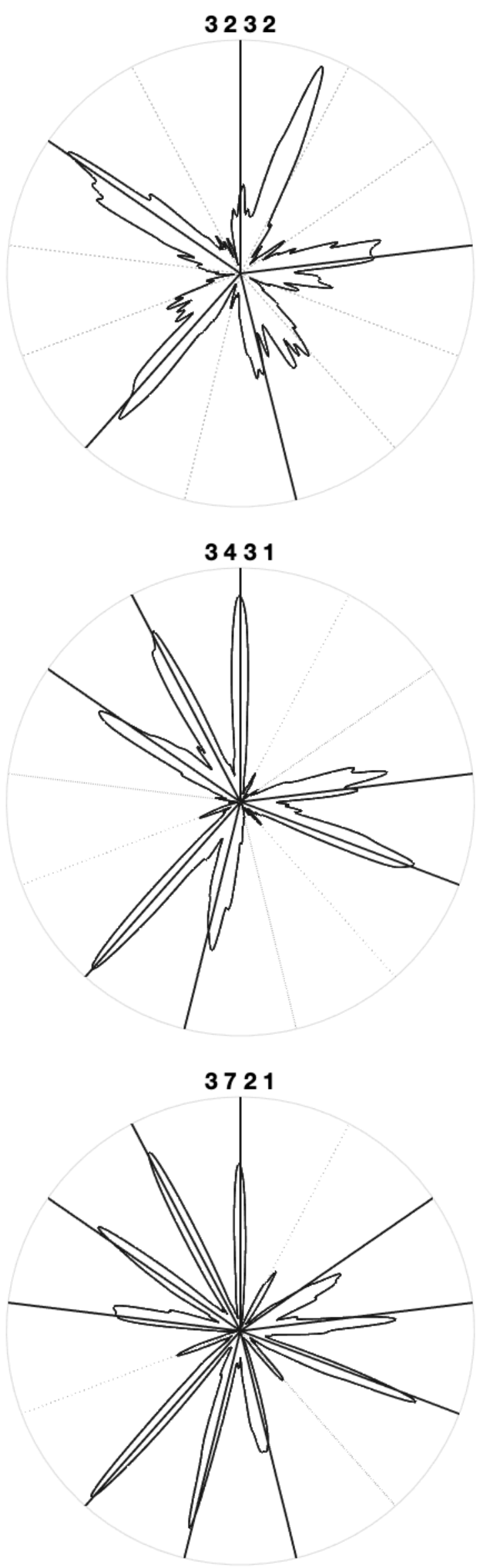
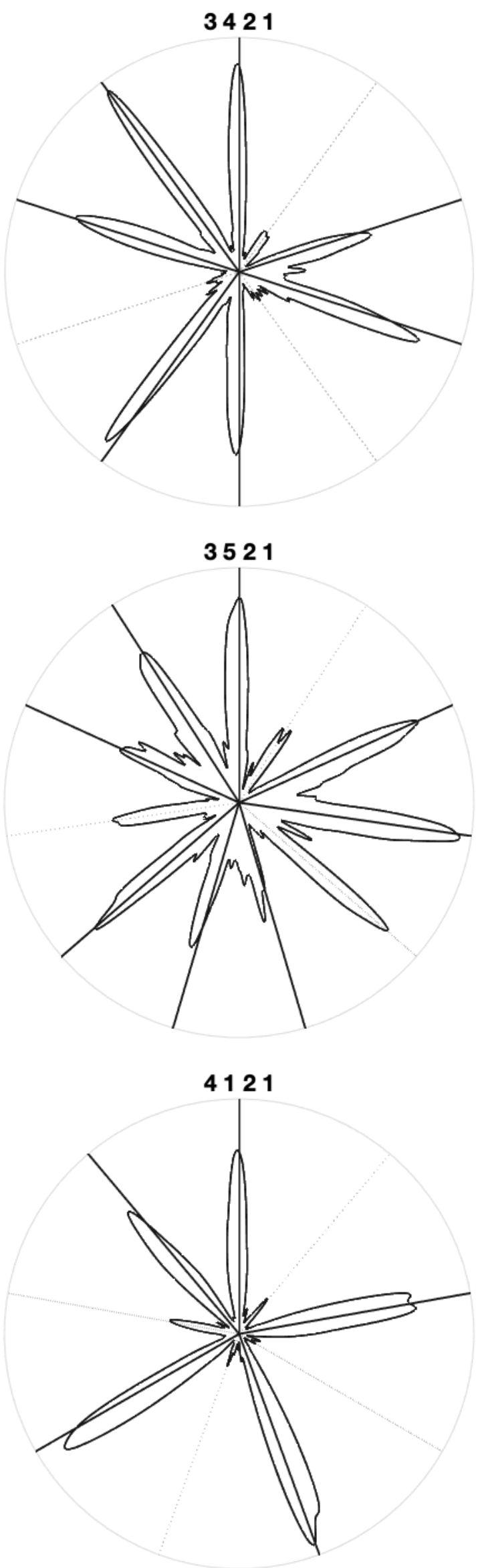

61 


$$
\begin{aligned}
& * * \\
& * * \\
& * *
\end{aligned}
$$




$$
\text { * }
$$




\section{A. 7 Performance-level tap accuracy model}

Table 9. Full summary of the tap accuracy model.

Family: beta

Links: $\mathrm{mu}=$ logit; phi = identity

Formula: similarity perf num + repetition + mean IOI + evenness + IOI ent + balance + duple triple +

(perf_num + repetition + mean_IOI + evenness + IOI_ent + balance + duplē_triple | participant) + (perf_num + repetition | rhythm)

Data: WF_rhythm_data_perf (Number of observations: 4975)

Samples: 4 chains, each with iter $=4500$; warmup $=2000$; thin $=1$;

total post-warmup samples $=10000$

Group-Level Effects:

participant (Number of levels: 111)

sd( Intercept)

Estimate Est.Error 1-95\% CI u-95\% CI Rhat Bulk_ESS Tail_ESS

sd(perf_num)

sd(repetition)

0.38

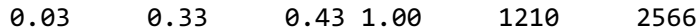

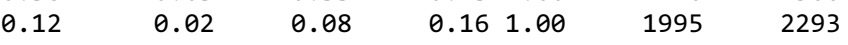

sd(mean_IOI)

sd(evenness)

0.02

$\begin{array}{lll}0.14 & 0.02 & 0.12\end{array}$

$\begin{array}{lll}0.05 & 0.03 & 0.00\end{array}$

$0.111 .00 \quad 1403 \quad 1602$

sd(IOI_ent)

0.07

$0.01 \quad 0.05$

$0.181 .00 \quad 3458 \quad 6216$

sd(balance)

0.10

$0.02 \quad 0.05$

$0.111 .00 \quad 1598 \quad 3263$

sd(duple_triple1)

0.14

$0.02 \quad 0.09$

0.101 .00

$3251 \quad 4159$

cor(Intercept,perf_num)

cor(Intercept, repetition)

$-0.13$

$0.14 \quad-0.39$

$0.141 .00 \quad 804 \quad 1666$

0.40

$0.18 \quad 0.03$

cor(perf_num, repetition)

$-0.71$

$0.18-0.92$

$-0.20$

$0.11-0.42$

0.181 .00

2160

$0.151 .00 \quad 4267$

$0.731 .00 \quad 4187$

$-0.251 .00$

0.021 .00

cor (perf_num, mean_IOI)

cor(repetition, mean_IOI)

0.01

$0.16-0.30$

0.311 .00

$2820 \quad 2658$

$-0.14$

$0.21-0.55$

0.281 .02

0.06

$0.28-0.50$

0.591 .00

$1424 \quad 3059$

cor (perf_num, evenness)

cor (repetition, evenness)

$-0.26$

$0.31-0.76$

$\begin{array}{lll}0.24 & 0.31 & -0.43\end{array}$

cor(mean_IOI, evenness)

cor (Intercept, IOI_ent)

$\begin{array}{rrr}-0.05 & 0.29 & -0.60 \\ 0.26 & 0.14 & -0.02\end{array}$

0.411 .00

0.771 .00

0.521 .00

0.531 .00

0.13

$0.19-0.25$

0.10

$0.24-0.36$

0.501 .00

0.561 .00

0.121 .00

cor (mean_IOI, IOI_ent)

cor (evenness, IOI_ent)

cor (Intercept, balance)

$-0.20$

$0.16-0.50$

$\begin{array}{lll}-0.01 & 0.30 & -0.59\end{array}$

$\begin{array}{lll}-0.18 & 0.16 & -0.50\end{array}$

0.581 .00

0.141 .00

0.591 .00

0.481 .00

$\begin{array}{ll}0.43 & 1.00 \\ 0.52 & 1.00\end{array}$

0.06

$0.25-0.51$

0.751 .00

0.44

$\begin{array}{ll}0.19 & -0.31 \\ 0.34 & -0.75\end{array}$

$0.18 \quad 0.04$

cor(IOI ent balance)

0.42

$0.14 \quad 0.13$

0.691 .00

0.071 .00

0.721 .00

0.30

0.23

cor (perf num, duple triple1)

$-0.33$

$0.16-0.62$

$-0.011 .00$

$522 \quad 1189$

$7729 \quad 7013$

$4667 \quad 6457$

$3727 \quad 5807$

$8308 \quad 7134$

$5909 \quad 7126$

$2457 \quad 4831$

$1197 \quad 2764$

$5587 \quad 7513$

$\begin{array}{lll}0.15 & 0.30 & -0.46\end{array}$

0.681 .00

0.431 .00

$923 \quad 2563$

$4771 \quad 4452$

$2516 \quad 4134$

$1446 \quad 3347$

$5433 \quad 6412$

$0.04 \quad 0.20 \quad-0.35$

$0.21 \quad-0.64$

0.201 .00

774

2421

3588

2213

2384

4134

4418

5081

2253

$4135 \quad 5895$

$1093 \quad 3502$

cor(IOI_ent, duple_triple1)

cor(balānce, duple_triple1)

$-0.23$

3157

5420

rhythm (Number of levels: 91)

$\begin{array}{lrrrrrr} & \text { Estimate } & \text { Est.Error } & 1-95 \% \text { CI } & \text { u-95\% CI } & \text { Rhat Bulk_ESS Tail_ESS } \\ \text { sd(Intercept) } & 0.23 & 0.02 & 0.19 & 0.271 .00 & 2118 & 4284 \\ \text { sd(perf_num) } & 0.05 & 0.02 & 0.01 & 0.091 .00 & 1629 & 1781 \\ \text { sd(repetition) } & 0.03 & 0.02 & 0.00 & 0.071 .00 & 1382 & 2978 \\ \text { cor(Intercept, perf_num) } & -0.29 & 0.31 & -0.83 & 0.381 .00 & 2802 & 3995 \\ \text { cor(Intercept, repetition) } & -0.25 & 0.38 & -0.88 & 0.621 .00 & 4923 & 4736 \\ \text { cor(perf_num, repetition) } & -0.17 & 0.49 & -0.89 & 0.811 .00 & 2633 & 4809\end{array}$

Population-Level Effects:

$\begin{array}{lrrrrrrr} & \text { Estimate } & \text { Est. Error } & 1-95 \% \text { CI } & \text { u-95\% CI } & \text { Rhat } & \text { Bulk_ESS Tail_ESS } \\ \text { Intercept } & -1.36 & 0.05 & -1.46 & -1.26 & 1.01 & 845 & 1766 \\ \text { perf_num } & 0.10 & 0.02 & 0.07 & 0.141 .00 & 4181 & 5641 \\ \text { repetition } & -0.01 & 0.02 & -0.04 & 0.02 & 1.00 & 4783 & 5524 \\ \text { mean_IOI } & -0.41 & 0.03 & -0.47 & -0.35 & 1.00 & 1928 & 3770 \\ \text { evenness } & 0.16 & 0.08 & 0.01 & 0.301 .01 & 1847 & 3520 \\ \text { IOI_ent } & 0.00 & 0.03 & -0.05 & 0.061 .00 & 1892 & 3744 \\ \text { balance } & -0.27 & 0.08 & -0.43 & -0.11 & 1.01 & 1706 & 3226 \\ \text { duple_triple1 } & 0.21 & 0.05 & 0.10 & 0.31 & 1.00 & 1708 & 3373\end{array}$

Family Specific Parameters: 


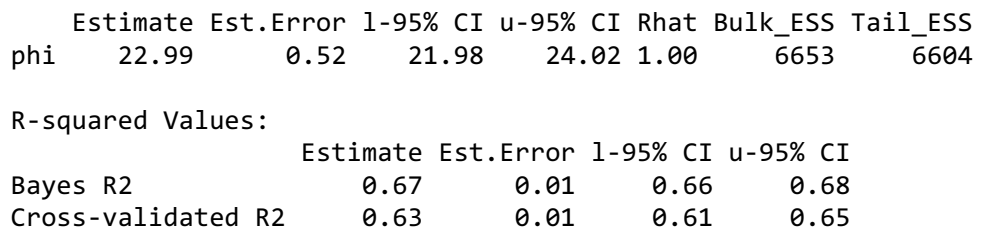

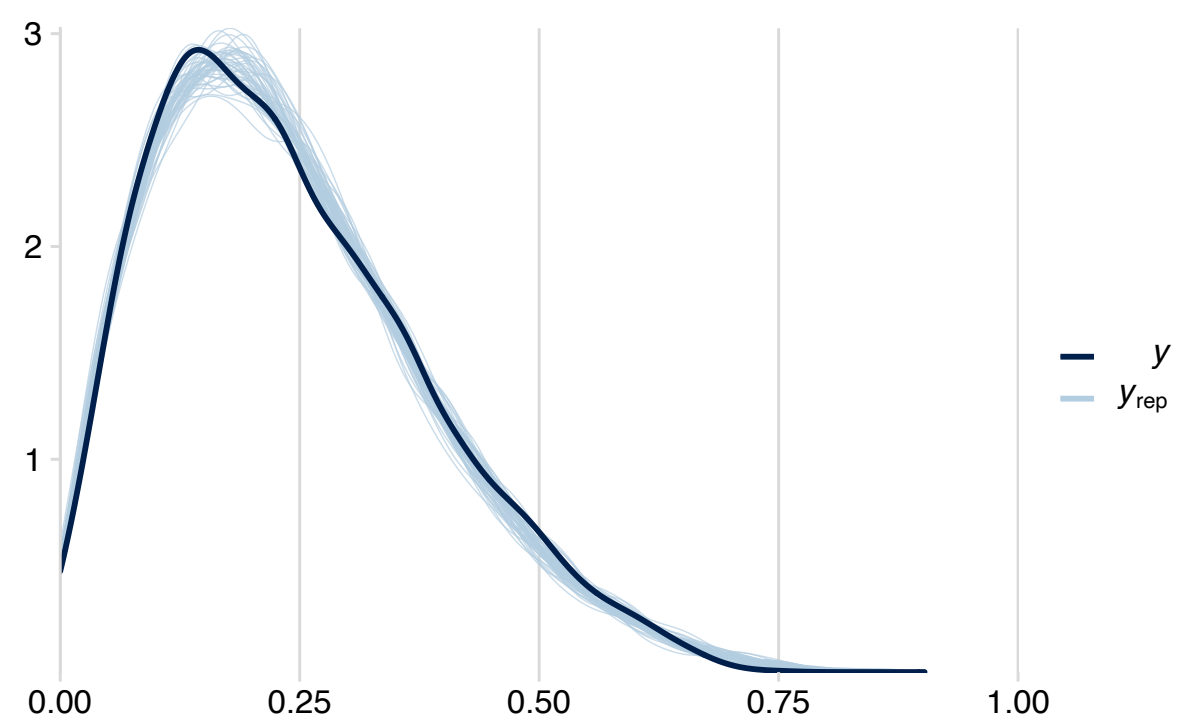

Figure 6. Posterior predictive check for the model shown above: $y$ is the observed data shown as a density plot; $y_{\text {rep }}$ shows 50 sets of corresponding predictions from the model. 


\section{A. 8 Pulse-level tap probability model}

Table 10. Full summary of the tap probability model.

Family: beta_binomial2

Links: $\mathrm{mu}=$ logit; phi = identity

Formula: tap | trials(n pulses) cue * (tap lag1 + perf num + repetition + N + mean IOI + CQ + balance + proj_cent + height + duple_triple + Markov $2+\bar{e}$ dge $)+($ cue | participant) + (cue | rhythm)

Data: WF_rhythm_data_pulse (Number of observations: 48282)

Samples: 4 chains, each with iter = 4500; warmup = 2000; thin = 1; total post-warmup samples $=10000$

Group-Level Effects:

participant (Number of levels: 111)

\begin{tabular}{|c|c|c|c|c|c|c|c|}
\hline & Estimate & Est.Error & $1-95 \% \mathrm{CI}$ & $\mathrm{u}-95 \% \mathrm{CI}$ & Rhat & ESS & 11 \\
\hline$d$ (Intercept) & 0.70 & 0.05 & 0.61 & 0.80 & 1.01 & 538 & 11 \\
\hline & 0.78 & 0.05 & 0.68 & 0.89 & 1.01 & 549 & \\
\hline rcept, cue1) & -0.89 & 0.02 & -0.93 & -0.85 & 1.00 & 652 & \\
\hline
\end{tabular}

rhythm (Number of levels: 91)

\begin{tabular}{|c|c|c|c|c|c|c|c|}
\hline & Estimate & Est.Error & $1-95 \% \mathrm{CI}$ & $\mathrm{u}-95 \% \mathrm{CI}$ & Rnat & Bulk_ESS & 1 \\
\hline ntero & 0.64 & 0.05 & 0.55 & 0.75 & 1.00 & 806 & \\
\hline & 0.96 & 0.07 & 0.82 & 1.12 & 1.00 & 773 & \\
\hline rcept, cue1) & -0.97 & 0.01 & -0.98 & -0.96 & 1.00 & 1198 & \\
\hline
\end{tabular}

Population-Level Effects:

\begin{tabular}{|c|c|c|c|c|c|c|c|}
\hline & Estimate & Est.Error & $1-95 \%$ CI & $\mathrm{U}-95 \% \mathrm{CI}$ & Rhat & Bulk_ESS & Tail_ESS \\
\hline Intercept & -1.27 & 0.12 & -1.51 & -1.06 & 1.00 & 472 & 917 \\
\hline cue1 & 2.02 & 0.16 & 1.71 & 2.34 & 1.01 & 476 & 930 \\
\hline tap_lag1 & -0.53 & 0.04 & -0.61 & -0.46 & 1.00 & 2884 & 4399 \\
\hline erf_num & 0.01 & 0.02 & -0.02 & 0.05 & 1.00 & 2123 & 4050 \\
\hline epetition & -0.08 & 0.02 & -0.11 & -0.05 & 1.00 & 2163 & 411 \\
\hline $\mathrm{N}$ & -0.19 & 0.08 & -0.36 & -0.03 & 1.01 & 543 & 1233 \\
\hline ean_IOI & -0.37 & 0.08 & -0.53 & -0.23 & 1.01 & 561 & 890 \\
\hline 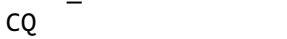 & 0.18 & 0.09 & 0.01 & 0.35 & 1.00 & 883 & 1837 \\
\hline alance & 0.12 & 0.10 & -0.08 & 0.32 & 1.01 & 598 & 1377 \\
\hline roj_cent & 0.45 & 0.02 & 0.41 & 0.48 & 1.00 & 3494 & 5512 \\
\hline ight & -0.05 & 0.01 & -0.07 & -0.02 & 1.00 & 3087 & 4847 \\
\hline uple_triple1 & -0.32 & 0.15 & -0.60 & -0.03 & 1.00 & 642 & 1228 \\
\hline arkov2 & 0.30 & 0.01 & 0.27 & 0.33 & 1.00 & 3836 & 5868 \\
\hline Age & 0.43 & 0.01 & 0.40 & 0.45 & 1.00 & 3015 & 5354 \\
\hline e1:tap_lag1 & 0.40 & 0.05 & 0.31 & 0.49 & 1.00 & 2984 & 4779 \\
\hline e1:perf_num & 0.17 & 0.02 & 0.13 & 0.20 & 1.00 & 2152 & 3981 \\
\hline de1:repetition & 0.07 & 0.02 & 0.04 & 0.11 & 1.00 & 2116 & 392 \\
\hline le1:N & 0.18 & 0.13 & -0.06 & 0.42 & 1.01 & 527 & 1320 \\
\hline e1:mean_IOI & 0.03 & 0.11 & -0.19 & 0.27 & 1.01 & 586 & 95 \\
\hline e1:CQ & -0.24 & 0.13 & -0.49 & 0.01 & 1.00 & 910 & 181 \\
\hline e1:balance & -0.16 & 0.15 & -0.45 & 0.13 & 1.01 & 588 & 122 \\
\hline le1:proj_cent & -0.22 & 0.02 & -0.26 & -0.18 & 1.00 & 3821 & 598 \\
\hline le1: height & 0.07 & 0.01 & 0.05 & 0.10 & 1.00 & 3020 & 4594 \\
\hline e1:duple_triple1 & 0.46 & 0.21 & 0.04 & 0.88 & 1.01 & 635 & 1227 \\
\hline e1:Markov2 & -0.27 & 0.02 & -0.31 & -0.23 & 1.00 & 4371 & 607 \\
\hline de1:edge & -0.64 & 0.02 & -0.67 & -0.61 & 1.00 & 3319 & 542 \\
\hline
\end{tabular}

Family Specific Parameters:

Estimate Est.Error 1-95\% CI u-95\% CI Rhat Bulk_ESS Tail_ESS

$\begin{array}{llllllll}\text { phi } & 9.78 & 0.13 & 9.54 & 10.04 & 1.00 & \overline{9} 118 & \overline{7} 454\end{array}$

R-squared Values:

$\begin{array}{lrrrr} & \text { Estimate } & \text { Est. Error } & 1-95 \% \text { CI } & \text { u-95\% CI } \\ \text { Bayes R2 } & 0.85 & 0.00 & 0.84 & 0.85 \\ \text { Cross-validated R2 } & 0.85 & 0.00 & 0.85 & 0.86\end{array}$




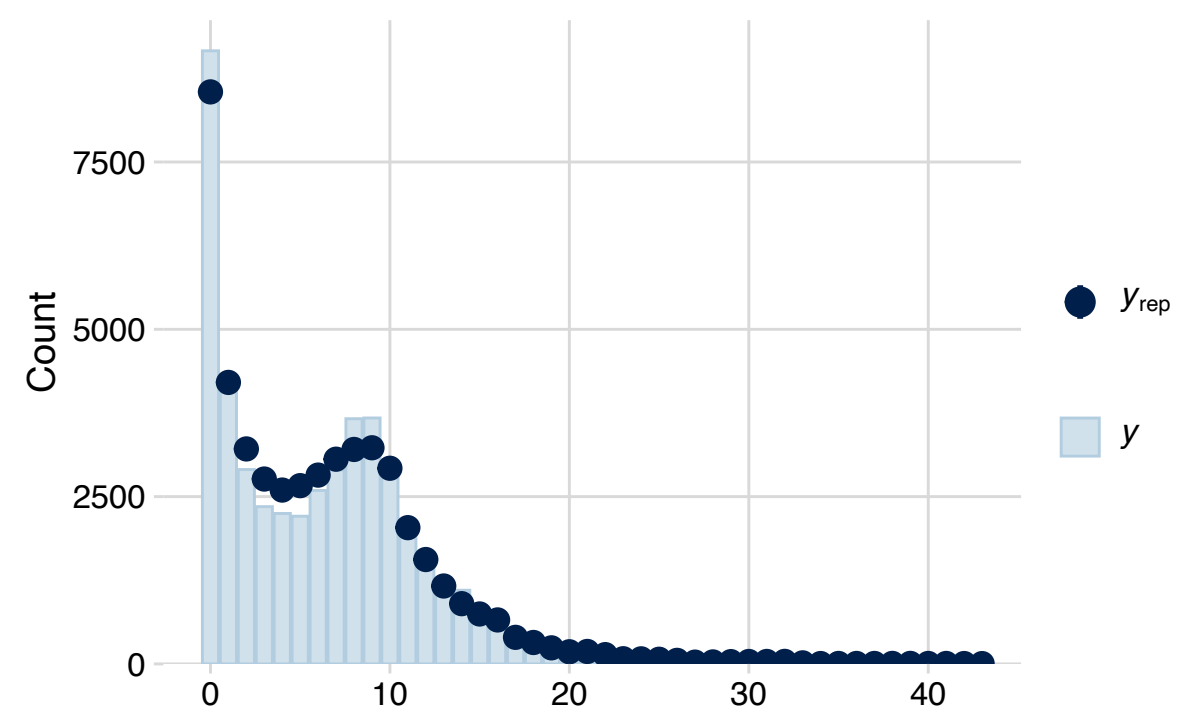

Figure 7. Posterior predictive check for the model shown above: $y$ is the observed data shown as a histogram; $y_{\text {rep }}$ shows the $90 \%$ intervals obtained from 50 sets of corresponding predictions from the model. The observed data is, for each periodic pulse in each performance, the number of times it was tapped.

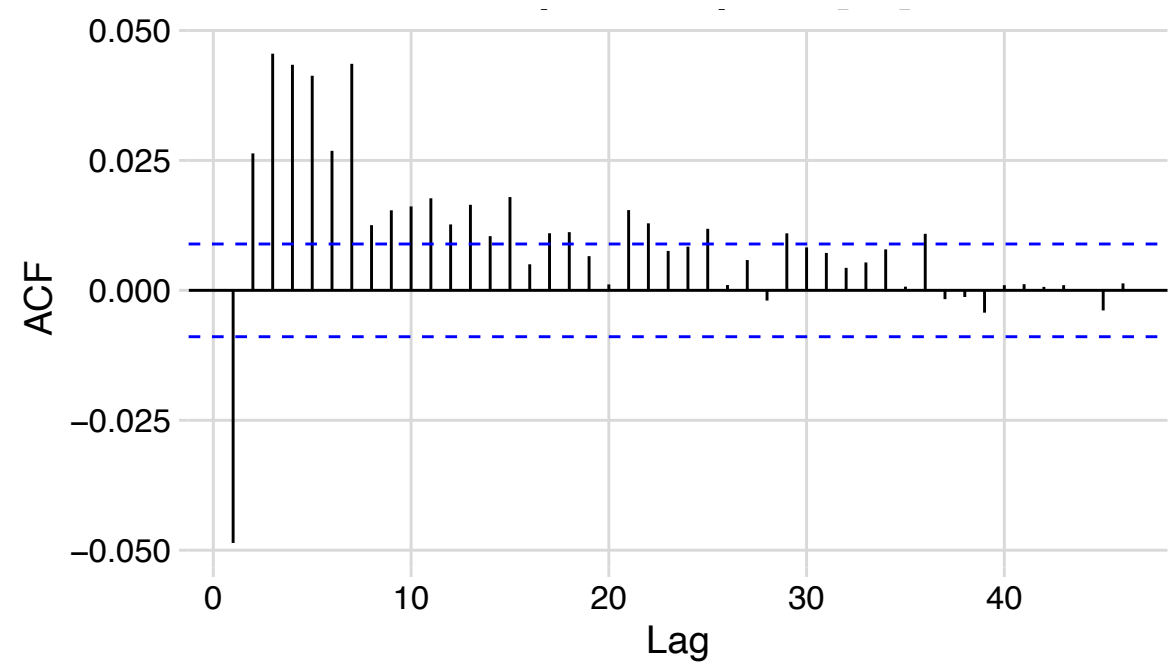

Figure 8. Autocorrelations of the tap probability model's residuals.

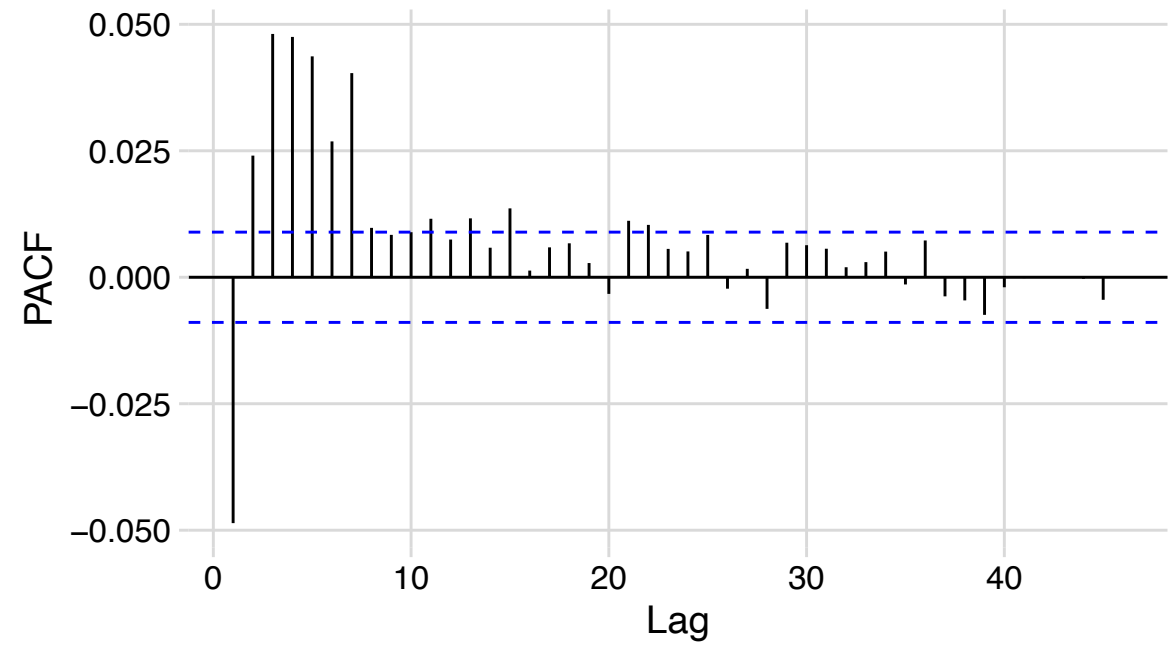

Figure 9. Partial autocorrelations of the tap probability model's residuals. 


\section{A. 9 Pulse-level cued pulses with Povel predictors}

Table 11. Full summary of the tap probability model for cued pulses substituting Povel's predictors (iso_accent, end_accent, and start_accent) for edge.

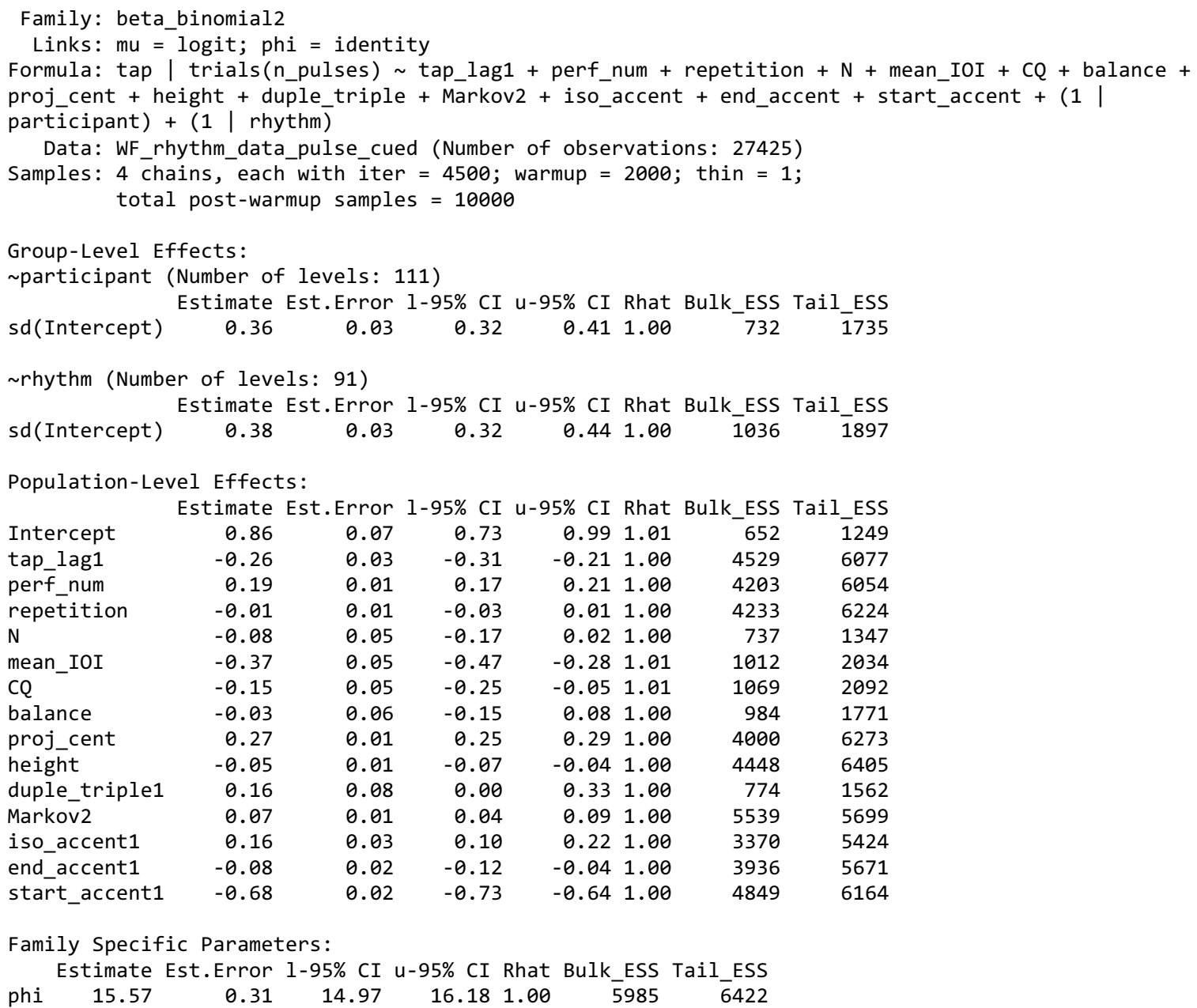

Figure 10. Posterior predictive check for the model shown above: $y$ is the observed data shown as a histogram; $y_{\text {rep }}$ shows the $90 \%$ intervals obtained from 50 sets of corresponding predictions from the model. The observed data is, for each cued periodic pulse in each performance, the number of times it was tapped. Note that the histogram is different to Figure 7 because this model includes only cued pulses. 


\section{A. 10 Pulse-level cued pulses with edge predictor}

Table 12. Full summary of the tap probability model for cued pulses using edge instead of Povel's predictors.
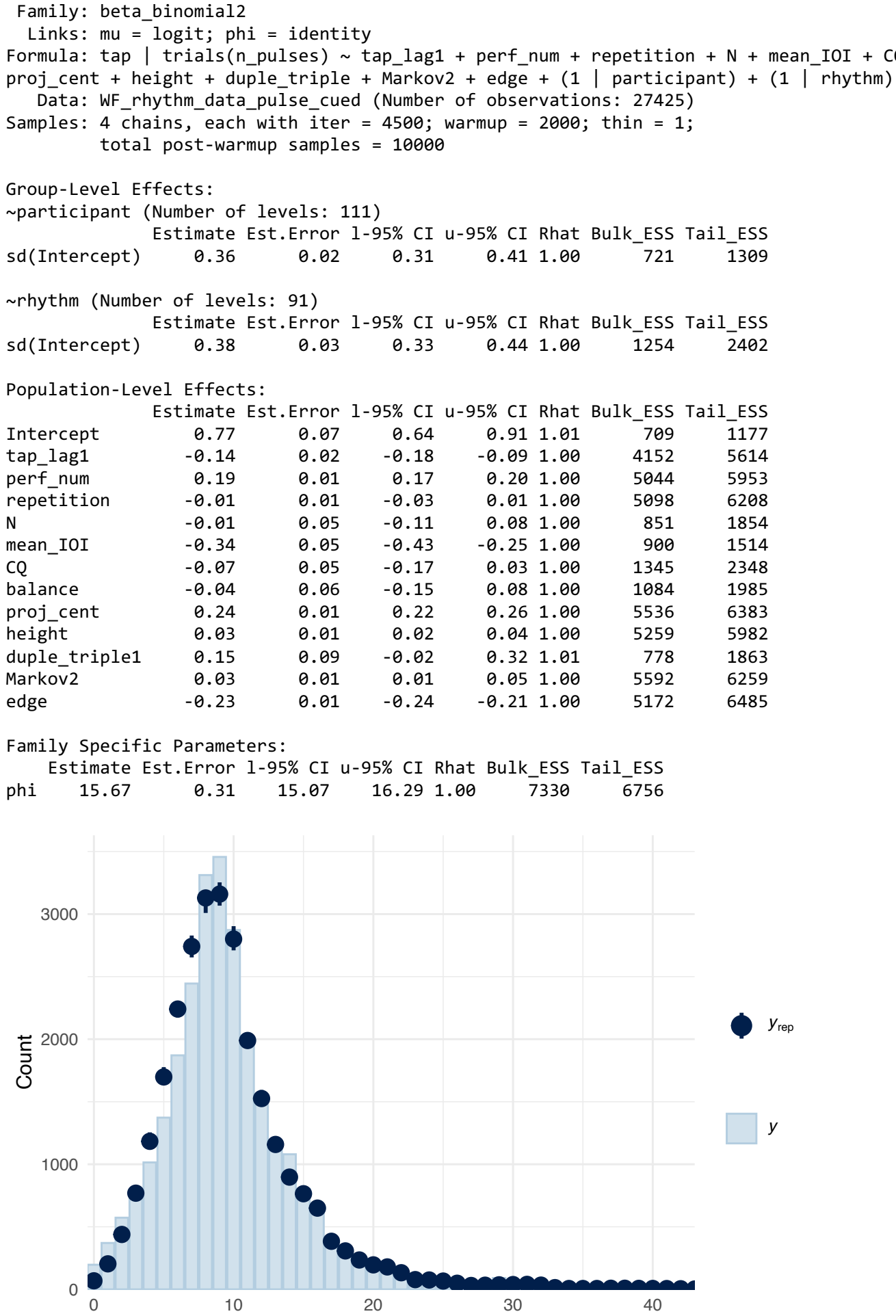

Figure 11. Posterior predictive check for the model shown above: $y$ is the observed data shown as a histogram; $y_{\text {rep }}$ shows the $90 \%$ intervals obtained from 50 sets of corresponding predictions from the model. The observed data is, for each cued periodic pulse in each performance, the number of times it was tapped. Note that the histogram is different to Figure 7 because this model includes only cued pulses. 


\section{A. 11 Pulse-level velocity model}

Table 13. Full summary of the tap velocity model.

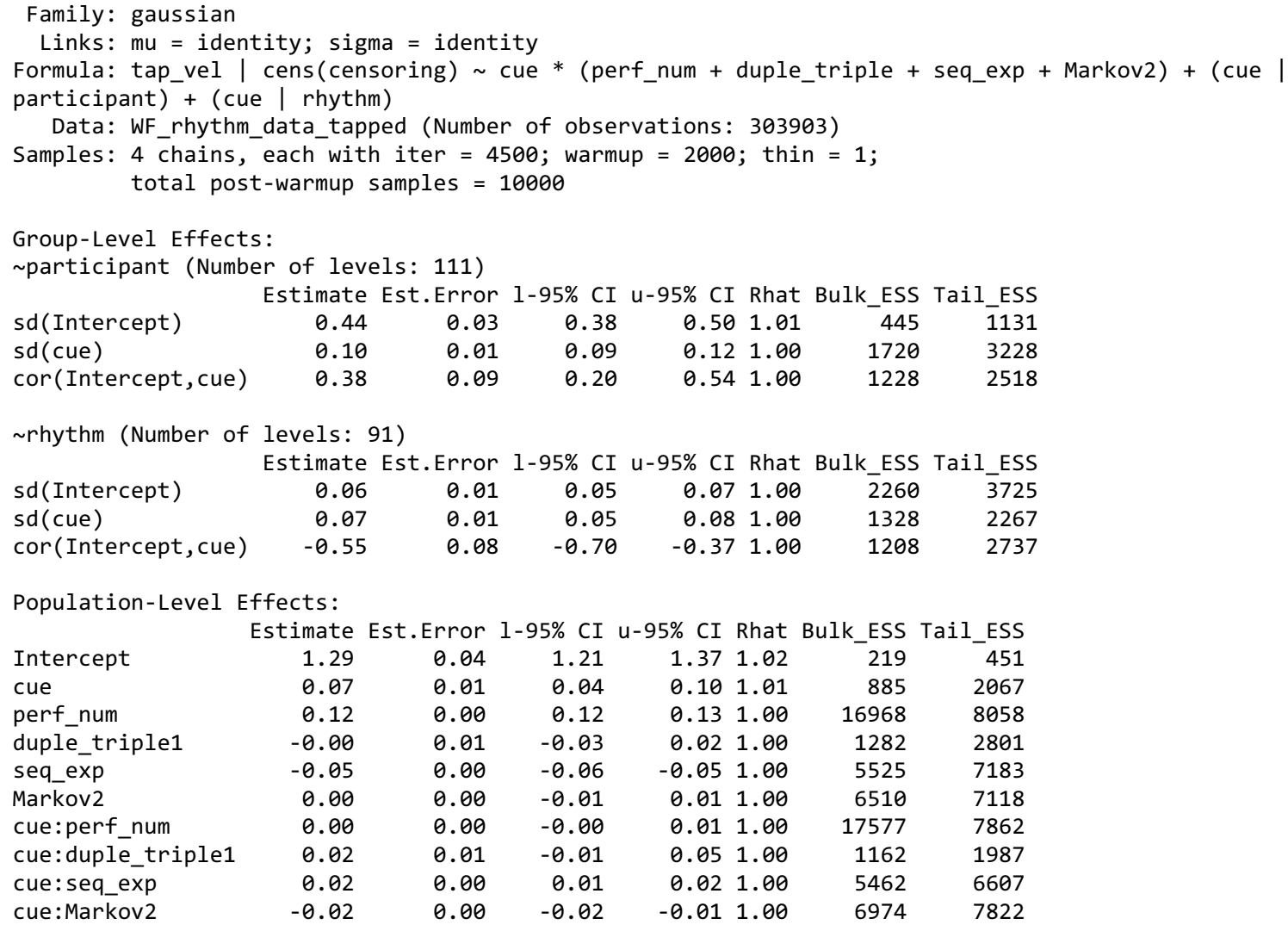

\begin{tabular}{|c|c|c|c|c|c|c|c|}
\hline & Estimate & Est.Error & $1-95 \%$ CI & $\mathrm{u}-95 \% \mathrm{CI}$ & Rhat & Bulk_ESS & il_ESS \\
\hline Intercept & 1.29 & 0.04 & 1.21 & 1.37 & 1.02 & 219 & 451 \\
\hline cue & 0.07 & 0.01 & 0.04 & 0.10 & 1.01 & 885 & 2067 \\
\hline perf_num & 0.12 & 0.00 & 0.12 & 0.13 & 1.00 & 16968 & 8058 \\
\hline duple_triple1 & -0.00 & 0.01 & -0.03 & 0.02 & 1.00 & 1282 & 2801 \\
\hline seq_exp & -0.05 & 0.00 & -0.06 & -0.05 & 1.00 & 5525 & 7183 \\
\hline Markov2 & 0.00 & 0.00 & -0.01 & 0.01 & 1.00 & 6510 & 7118 \\
\hline cue:perf_num & 0.00 & 0.00 & -0.00 & 0.01 & 1.00 & 17577 & 7862 \\
\hline cue:duple_triple1 & 0.02 & 0.01 & -0.01 & 0.05 & 1.00 & 1162 & 1987 \\
\hline cue:seq_exp & 0.02 & 0.00 & 0.01 & 0.02 & 1.00 & 5462 & 6607 \\
\hline cue:Markov2 & -0.02 & 0.00 & -0.02 & -0.01 & 1.00 & 6974 & 7822 \\
\hline
\end{tabular}

Family Specific Parameters:

R-squared Values:

$\begin{array}{lrrrr} & \text { Estimate } & \text { Est. Error } & 1-95 \% \text { CI } & \text { u-95\% CI } \\ \text { Bayes R2 } & 0.62 & 0.00 & 0.62 & 0.62 \\ \text { Cross-validated R2 } & 0.58 & 0.00 & 0.58 & 0.58\end{array}$

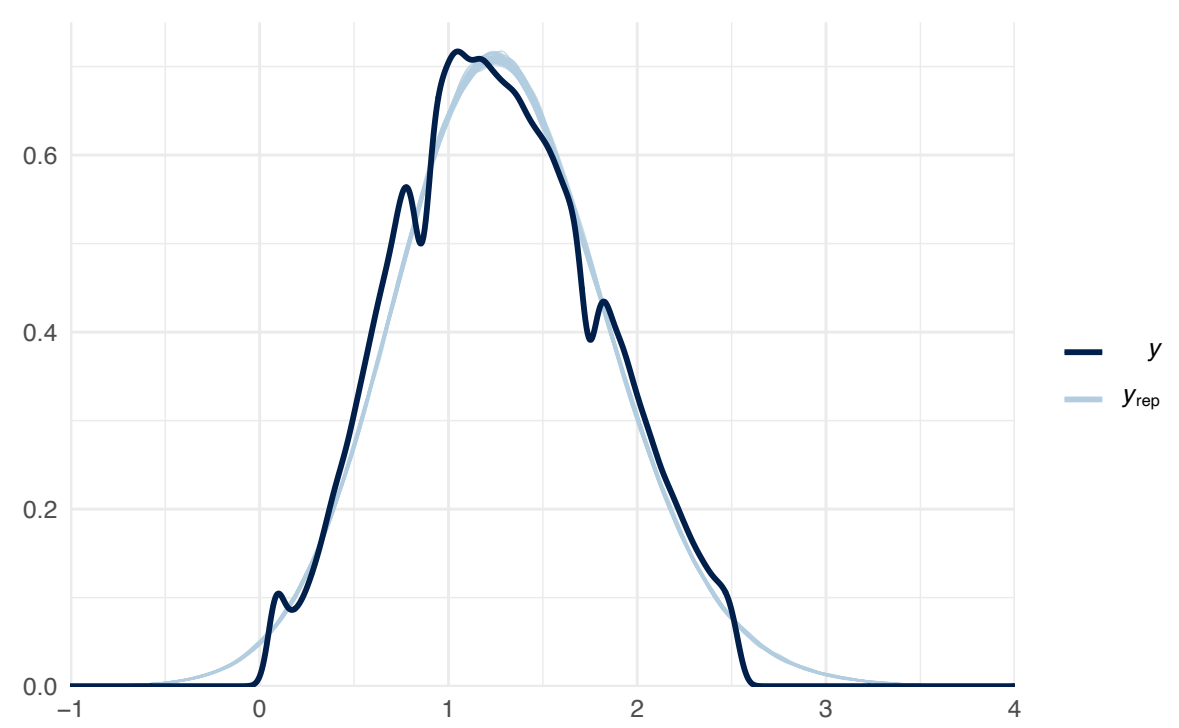

Figure 12. Posterior predictive check for the censored model shown above: $y$ is the observed data shown as a density plot; $y_{\text {rep }}$ shows 50 sets of corresponding predictions from the model. 


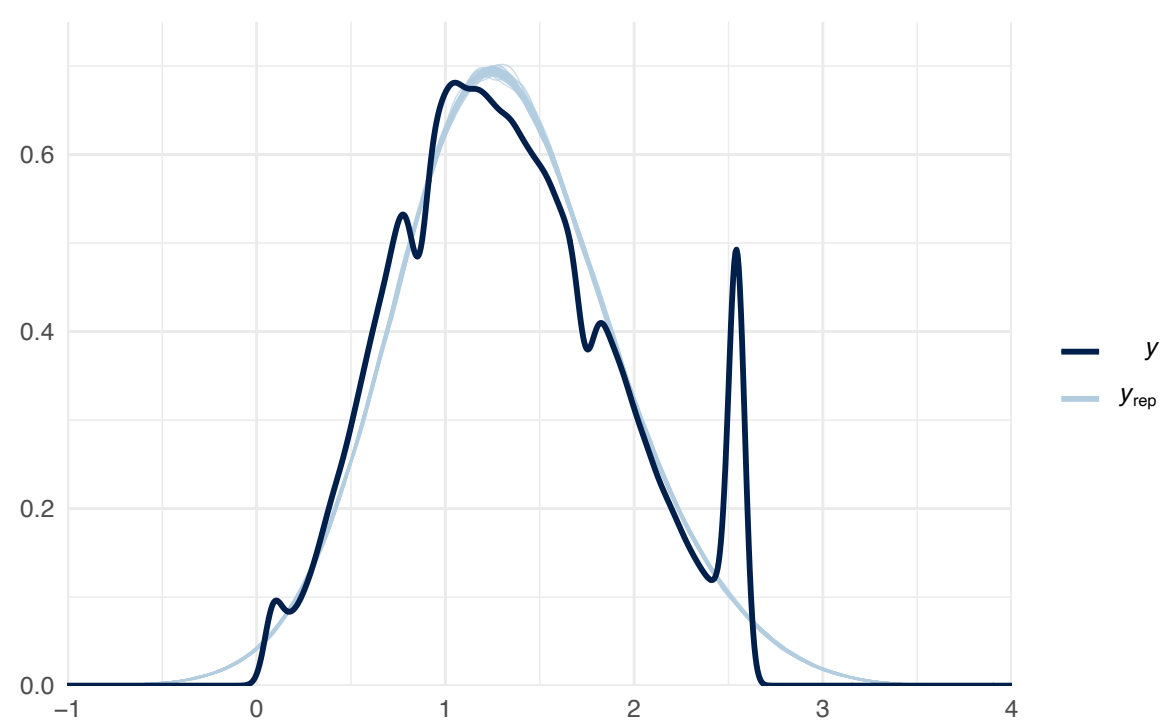

Figure 13. For comparison, this is the posterior predictive check obtained when censoring is not applied: $y$ is the observed data shown as a density plot; $y_{\text {rep }}$ shows 50 sets of corresponding predictions from the model. The peak in the data at the right results from the cluster of values at the maximum MIDI velocity value of 127 . Many of these taps at 127 would have been harder than the minimum required to reach this velocity value. Note how in the above plot from the censored model, these taps are no longer modelled as being at 127; rather they are modelled as if they represent values $\geq 127$, and so are no longer directly depicted in the plot. 


\section{A. 12 Pulse-level tap-timing error model}

Table 14. Full summary of the tap timing error model.

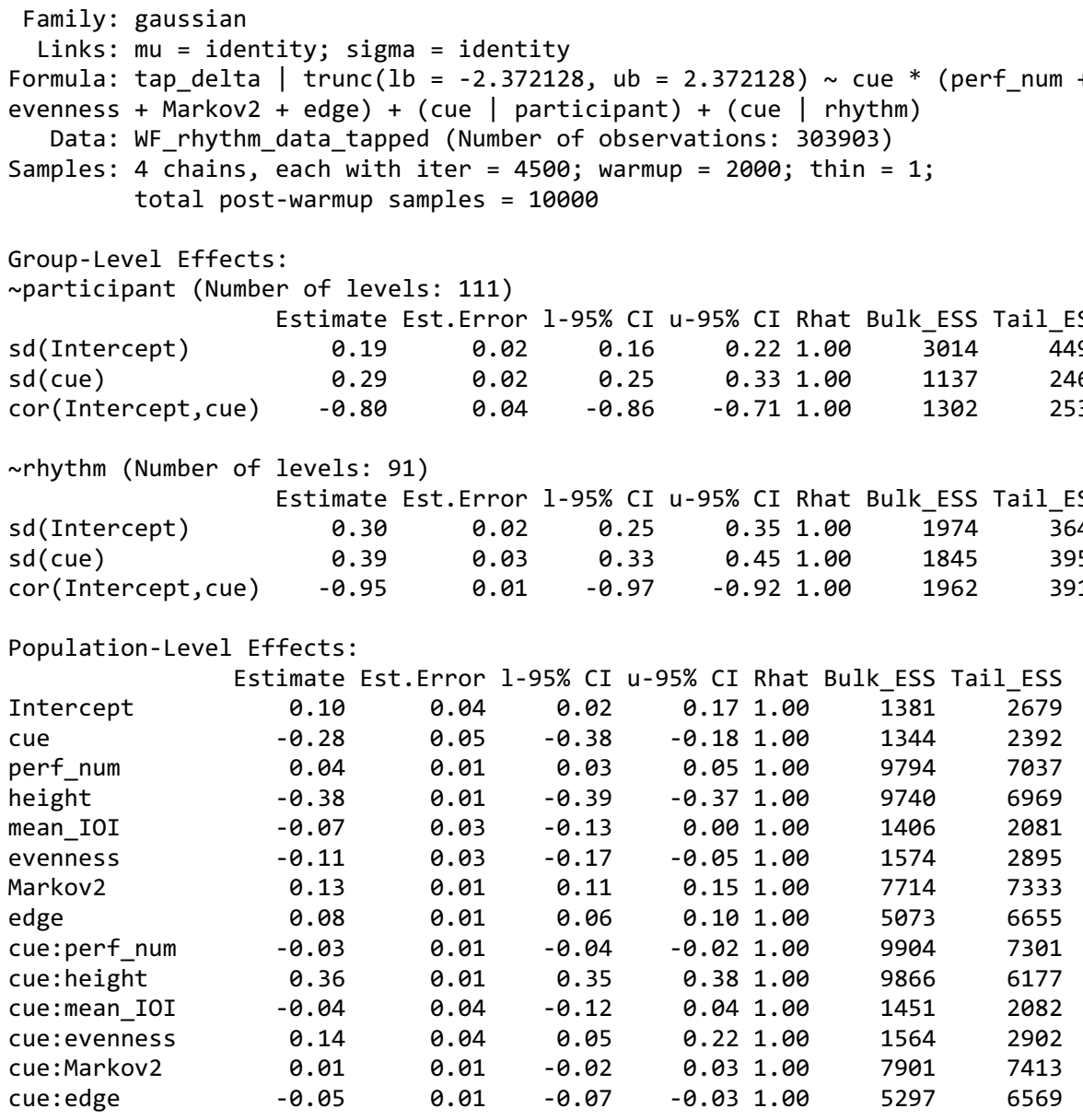

Family Specific Parameters:

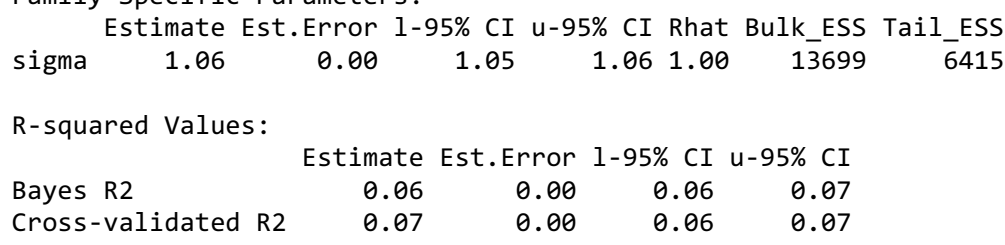

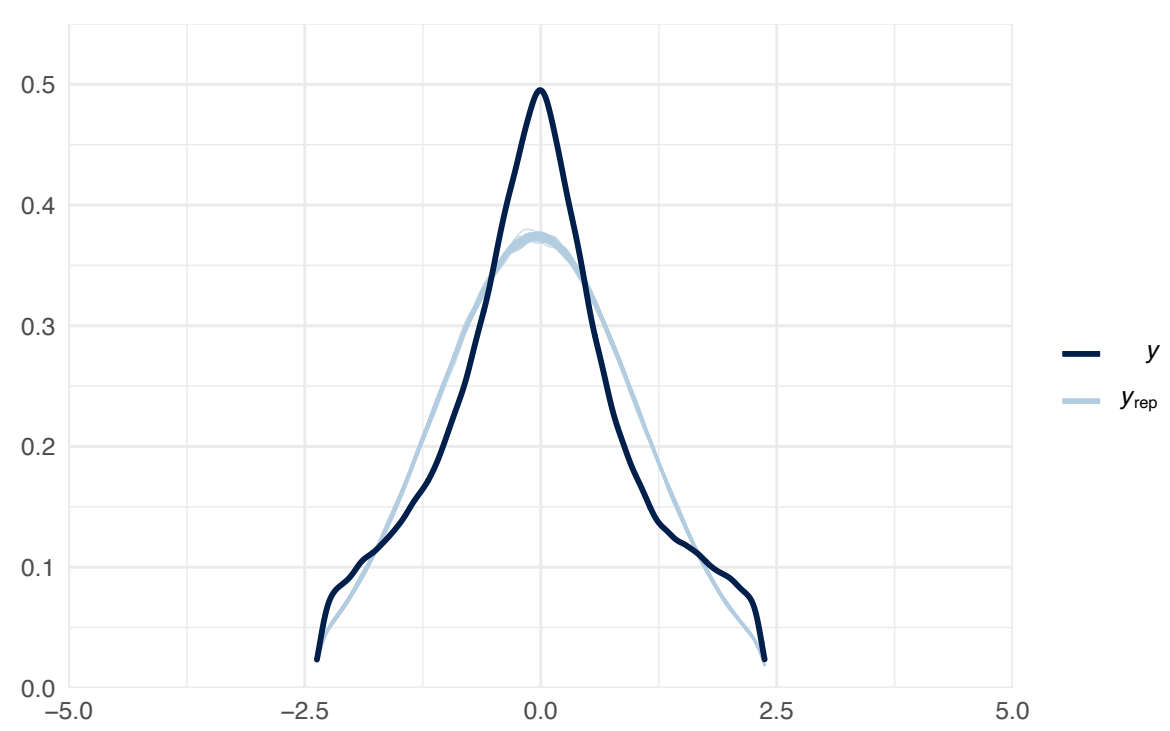

Figure 14. Posterior predictive check for the censored model shown above: $y$ is the observed data shown as a density plot; $y_{\text {rep }}$ shows 50 sets of corresponding predictions from the model. 


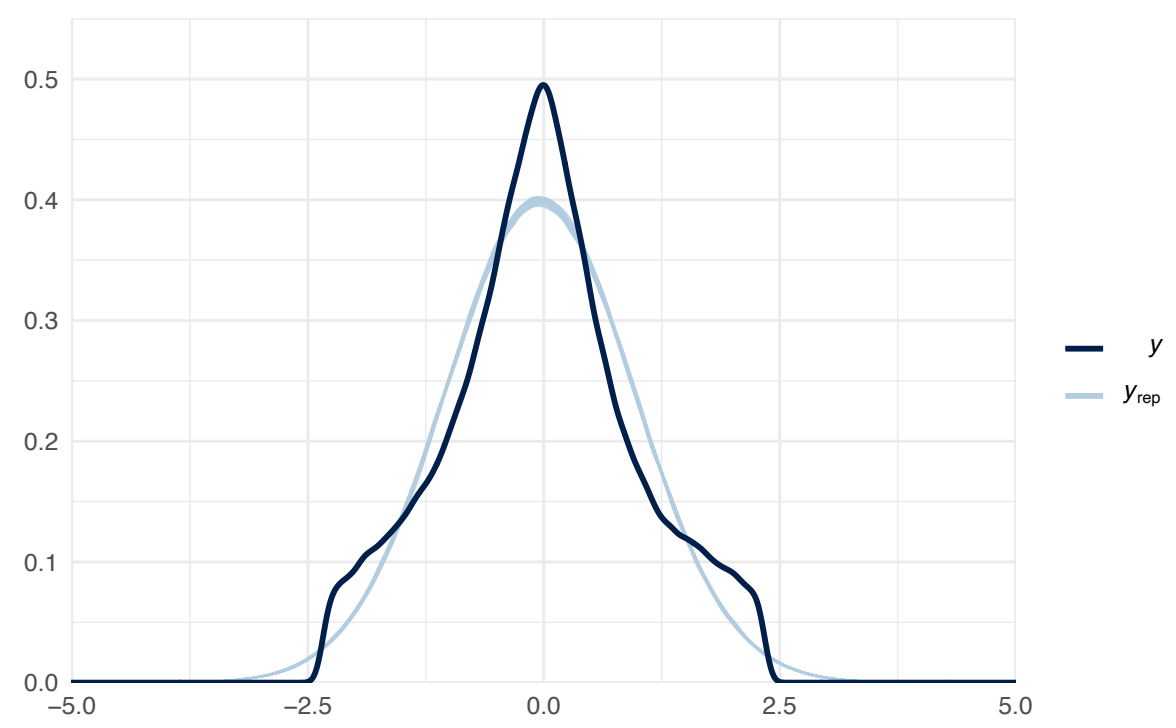

Figure 15. For comparison, this is the posterior predictive check obtained when truncation is not applied: $y$ is the observed data shown as a density plot; $y_{\text {rep }}$ shows 50 sets of corresponding predictions from the model. It is worth noting that there is some misfit in both the truncated and untruncated versions - prior to the truncation, the tails are very heavy. A student- $t$ distribution was attempted, but fitted worse. 